\title{
Colour Forecasting
}

Tracy Diane Cassidy, School of Art, Design and Architecture, University of Huddersfield, Huddersfield, UK

\author{
T.D.Cassidy@hud.ac.uk
}

\begin{abstract}
\end{abstract}
The underlying principles of colour forecasting, its purpose and the process, now have a history spanning a full century. While the trend-forecasting sector, as it is today, developed predominantly in the 1960s / 70s, the first known official colour forecasting agency, the Textile Color Card Association of America (TCCA), was established much earlier in 1915 - and is still in existence today, though it is now known as the Color Association of the United States (CAUS).

This issue of Textile Progress is somewhat timely as the challenges that industry face now differ from the challenges of the twentieth century, in particular, the increased need to address sustainability and the increased competiveness of the global marketplace. By providing an overview of the historical context, the importance of the role of colour forecasting in the fashion and textile industries is considered before setting the scene for engagement with the practice of colour forecasting, which is often referred to as being a combination of an art and a science due to the different techniques used and the different skill sets employed by forecasters to differentiate themselves from their competitors. It provides a detailed analysis of the more-recently established and establishing trend forecasting methods with a view to thinking more innovatively about the potential practice of colour forecasting. Furthermore, it investigates the effects and impact of colour and trend forecasting with a particular focus on the consequences of the process and system on business, on consumers and on the environment, drawing first on the theory of planned obsolescence and second on the sustainability of the system itself.

Reviewing the developments in colour forecasting begin-with the techniques that are considered to be the art of forecasting, followed by the colour forecasting process itself and the role it plays in the industry, including its application in textile design, garment design and product development, whilst highlighting the strengths and weaknesses of the different approaches. The more-objective characteristics of the colour forecasting process that have emerged from engagement with new developments and new thinking, provide a focus on the potential for greater accuracy in colour forecasting. This issue of Textile Progress explains how and why colour forecasting is an essential component of the business of making and selling fashion garments, through merchandising, retailing and fashion marketing, as well as being important in fashion design and product development processes. Developments affecting the design of colour-forecasting systems are shown to draw on marketing theory, without much consideration for the human-business interface, 
specifically, colour preferences, colour psychology and cultural meanings of colour. In addition, whilst forecasters claim to engage in research and include developments and new technologies as they materialise in the dyeing sector, there is little evidence in the literature of how precisely research and development within the industry has impacted, if at all, on colour forecasting, unless the developments have become generally newsworthy. The overall aim of this Textile Progress is to assist an understanding of the colour forecasting process and its contribution to the larger trend-forecasting system, and to highlight the challenges for the colour forecasting to face in twenty-first century fashion-business strategy. Attention is paid to the colour forecasting process, the art of forecasting and forecasting as a system establishing its role within the industry, including its application in the design process and skills acquisition, and the strengths and weaknesses of the current colour and trend forecasting process are presented. The more-recently established trendforecasting methods are critically analysed, as are previously unpublished contributions to knowledge through original sets of primary research data, and potential improvements are suggested.

Keywords: colour forecasting; trend forecasting; design process; product development; fashion retailing; planned obsolescence; sustainability.

\section{Introduction}

Technical developments in spinning, weaving, machine knitting, garment construction and manufacturing were the catalysts for the development of the ready-to-wear apparel industry in America from around the middle of the nineteenth century. The ready-to-wear sector had by then begun to emerge as mass production and the development of the retail sector, as we know it today, had developed in earnest, and by this time the processes of fabric manufacture and garment production were relatively cheap and fashionable products were more-widely available. By piecing together details about the textile and fashion industry over time the need for colour and trend forecasting can be identified and its importance in the fashion system today can be rationalised. There is evidence that throughout the period that followed the earliest stages in the mass production of clothing and fashion retail development, British textile and garment manufacturers had visited America during the nineteenth century, though this may have been more related to the growing competition due to the development of the American industry [1] than merely seeking inspirations for their own products. Trend-forecasting as it is today was developed predominantly in the 1960s and 1970s, as a result of changing consumer behaviour in the 1950s and 1960s supported by technological advances in textile and clothing manufacture. Consequently, according to Valan, as 'modernity penetrated daily life, colour became the symbol of change' [2], and the culmination of these factors promoted the acceptance, or the need for colour forecasting.

\subsection{Historical overview}

The textile industry developed sequentially through the inventions and refinements of spinning machinery in the early eighteenth century and these were instrumental both in increasing the speed of yarn production and improving the quality of the yarns; similar advances in powerloom technology 
improved both the quality and production rates of woven fabrics [3], whilst downstream, the development of the sewing machine in the mid-1850s offered opportunities to improve production rates in clothing manufacture. Competition increased in the textile industry and more spinning mills and fabric manufacturing units were opened by the growing number of entrepreneurial businessmen keen to profit from the industrial revolution.

The emergent wealth-class division enhanced by the industrial revolution became ever more apparent and the unwritten rules of social status, behaviour and appearance developed by earlier privileged classes were enhanced and became more-widespread. The wealthy were dressed by a rising number of designers, and as cheaper fabrics became more-readily available and affordable to a wider range of the population, distinctive style, cut and fit became more important in high fashion in order to distinguish the elite from the growing number of (less wealthy) followers of fashion [4]. One of the earliest designers at the time of the emergence of the industrial revolution was Rose Bertin who operated from her salon in Paris circa 1773 to 1793 . Her reputation essentially grew due to her rare talent for using colour together with ability to exploit style and fit in her designs; as an indication of her success, she later became personal dressmaker to Marie Antoinette [5]. By the early nineteenth century the factory system had been established for textiles and the first clothing factories began to appear, originally for making army uniforms; fashion change was still slow at this time [6], yet, by around 1825 British manufacturers were known to have been visiting America for design inspiration, providing an early indication of the need for some kind of forecasting system, at the very least in terms of fashion direction. This method involving sourcing inspiration from further afield is still a part of the forecasting system today.

By the mid-nineteenth century, technological advancements in the garment-manufacturing sector ensured higher production rates for apparel, though efforts aimed at improving the quality of clothing were still rudimentary, largely due to the challenges associated with achieving good fit for the mass population. At the same time the Parisian haute couture business, where style, cut, fit and the skill of the seamstress were paramount to high fashion, began to develop in earnest [7]. The couture industry was at this time the backbone of the fashion industry and a primary source of inspiration for garment manufacturers, and for the dressmakers who served the less-wealthy classes. The newly-developing retail sector, created through the establishment of department stores in major towns and cities across Britain, Europe and America, supported further developments in the apparel manufacturing sector. Partmade garments were displayed and completed in a bespoke manner for customers in-store and on demand, which helped the garment-manufacturing industry to cope in the short-term with the problem they were encountering with poor fit [8].

Great interest in colour and the prospect for the development of ranges of new synthetic dyes was stimulated by William Perkin's discovery of the first synthetic dye, Mauveine, in 1856 and the opening of his factory to mass-produce the dye in 1857. An international exhibition held in London in 1862 (a follow-on from the first-ever exhibition of manufactured products, namely the successful Great Exhibition held in London in 1851), exhibited the new colours available for textiles and fabrics [9]. Experiments aimed at the production of artificial silk in the mid-1800s culminated in the development of 
viscose rayon in 1894 by Cross and Bevan, opening up the possibility of more-affordable ranges of filament fabrics for use in garment making.

By the turn of the twentieth century, the more-innovative manufacturers of ready-to-wear apparel began to push the frontiers of mass-produced fashion by continually taking inspiration from the moreintricate couture ranges. This move towards more-stylish products gave impetus to the search for ways to improve the quality of the mass-produced garments; more-profitable marketing avenues were sought, which became essential as prosperity grew (at least for some) diversity in consumer lifestyles became apparent. It had become increasingly difficult for manufacturers to read and meet the needs and desires of the consumer, as by this time, according to Marcketti and Parsons [10], 'forecasting and managing fashion change presented a fundamental challenge to the burgeoning number of manufacturing and retailing associations formed to provide a structure to the developing industry'. It is not surprising therefore, that the Textile Color Card Association of America (TCCA) was established in 1915 to support the ready-to-wear industry; this had developed much earlier in America (c. 1830) than it had in Britain and Europe (mid-late $19^{\text {th }}$ Century). Even so, it is interesting to note that originally, colour forecasts were driven by the European Millinery industry, France in particular, and that TCCA came into existence following communication disruptions during World War I [11]. Regardless of the communications issue, while it had otherwise proved possible to copy the styles of the Parisian hautecouture designers relatively quickly, a need had nevertheless emerged for insight into colour direction earlier in the manufacturing process in order for the fibre, yarn and fabric manufacturers to prepare for the needs of garment manufacturers well ahead of the selling season [12].

\subsection{The first colour forecasting agency}

The aforementioned Textile Color Card Association of America (TCCA) was founded in 1915 as a nonprofit organisation in New York. The TCCA would appear to have been the first official agency of its kind to attempt to forecast seasonal colours for the fashion and textile industry. The agency's first forecast, which proposed colours in groups, was issued in 1917 for women's wear. The forecast was aimed at designers and stylists in the ready-to-wear sector in the USA, established well ahead of that in Britain and Europe. Inevitably, American mass-production apparel manufacturers felt a strong need for colour inspiration and direction.

TCCA changed its name in 1955 to the Color Association of the United States (CAUS) and this continues to be the name it uses today [13]. The organisation consists of apparel and textile professionals plus individuals from retail and education, and professionals from other sectors including interiors and the automobile industry. A committee for each sector was established comprising between five and ten specialists who meet biannually to determine seasonal colours for their sector, approximately two years ahead of a selling season. Subscribers to the organisation receive the seasonal colours as a forecast package comprising wool and cotton dyed threads, accompanied by a written report which gives the rationale for the colour selection, and sets of images to further support the concepts [14].

Colour forecasters operated within the confines of their own geographical region during the early twentieth century, possibly due to the relatively under-developed communications system of that time. 
The TCCA developed a format for their forecasts that would appeal to their intended users, American designers and stylists, in order to encourage acceptance of their colour directions. This format enabled the onlooker to easily recognise connections between the colours that made the overall palette look appealing. Through this format, designers would be able to intuitively understand how to read and use the forecasts. According to Walsh [15], the format 'also became the means of seduction by which others in non-fashion industries accepted subjective shade selections'. In order for the agency to remain in existence commercially, further forecasts were introduced in order to target other consumer-driven industries where colour is used as a sales and marketing tool and 'continues to enlist the volunteer efforts of industry professionals in reaching a consensus'.

\subsection{The need for forecasting in the early twentieth century}

According to Blaszczyk and Wubs [16], Lyon dye houses supplied companies such as J. Claude Frères \& Cie., with shade cards during the 1800 s that were repackaged into Paris trend reports to be retailed globally, and in the 1900's it was American millinery companies who systematised such colour forecasts for that country's textile industry. It would appear therefore that by the 1880 's, France had not only dominated direction of the fashion-couture industry, but also the then under-developed area of colour forecasting. Colour forecasting eventually migrated to America during the time of WWI as the States were then not easily able to source, in the established way, colour information from France or dyestuffs from Germany. Such independence appeared to have happened in the US prior to Britain and other European countries developing their own forecast information. The first acclaimed American colour forecaster is reputed to be Margaret Hayden Rorke, who joined the TCCA in 1918; Blaszczyk and Wubs [16] provide an example of a Swiss trade organisation's shade card - the Société de Teinture \& d'Apprêt, Basel - whose colour cards are said to have been used by Rorke for the development of her forecasts. It would therefore appear that in the early twentieth century, colour forecasts were really colour inspirations developed from the experimentation of dye manufacturers. It would of course be in their best interests to market their new colours to important parts of the textile and apparel industries.

By the beginning of the 1920's, many women in the UK and Western Europe had gained more freedom and independence following changes in their working lives during WWI. Following their experience of working in jobs once held only by men, and reinforced in the UK for example by winning the right to vote (but the right to vote only if you were well-off), many more women enjoyed new lifestyles and greater independence. Fashions became more synonymous with the different lifestyles available to them. New influences, such as music, dancing, sports and hobbies began to be used by designers for the creation of specific apparel items. Theatre became popular and costume designs became another important source of inspiration for everyday fashion [17]. Perhaps more importantly, marketing began to gain importance at this time and as the entertainment business grew the cinema, which was much more of a mass-participation activity than the theatre, and film-actresses in particular became excellent promoters of fashion. Although garment fit was still a challenge for the garment-manufacturing sector, the loose, straight silhouette popular at this time enabled completely-made garments to be morereadily available off-the-peg, whilst also offering a more-acceptable fit for the mass population. With changes such as this underway, the boutique established itself as an important retail concept for fashion 
which was later adopted by department stores [18]. Fashion was now also providing many new jobs for women, particularly as sales assistants and even in managerial roles in fashion establishments.

By the mid-1920's younger generations increased in their importance and influence on directing lifestyle and fashion. By now, colour print production techniques were cheaper, enabling more colour use in fashion magazines. Accurate colour and details became more important in disseminating the precise look to a mass market. Colour also offered a design element that could be changed quickly and easily by manufacturers to promote a new look. As Western women were increasingly enabled to display their own freedom and individuality, consumer demand was beginning to be felt as a driver by the fashion and textile industry. Parisian couturiers were now feeling their fashion leadership being threatened as bespoke creations became less important and even less profitable. Many of the fashion houses began to change their business models (in order to survive) by producing more affordable and marketable luxury products to appeal to the masses [19] and the large department stores began to employ fashion buyers to source innovative designs that would appeal to their store's clientele; such buyers needed insight into the demands of the consumer. Information about how consumer demand was changing and the rate at which it was changing began to gain more importance for the industry, as did the sources of such information. As a consequence, in 1927 Tobe Associates was established in America, a consultancy business focussed on fashion direction for manufacturers and increased its clientele during the early 1930s.

\subsubsection{The British Colour Council}

A similar organisation to the TCCA established in Britain in 1931, was The British Colour Council (BCC). This agency played a key role in Britain in forecasting seasonal colour palettes from fibre, yarn and fabric manufacturers to its subscribers [20]. The designers who worked in factory environments could not have the privilege of working on a one-to-one basis with clients as in the couture business and therefore had neither any control over convincing consumers of the 'right colour' for them this season, nor the ability to predict what consumers of their designs would like. More fashion-garment manufacturers were now seeking colour direction and began to invest more heavily in forecast packages, or, upstream from them like the weavers from Lyon, established promotional/marketing methods that included colour forecasting as one element of their efforts at influencing the market.

\subsection{The development of the forecasting sector in the mid-twentieth century}

In the mid-twentieth century, western countries including those in the wealthier and more-developed parts of Europe and the USA were taking the lead in the development of haute couture, in clothing manufacturing, and in off-the-peg garment retailing and therefore also in colour forecasting. By the end of WWII, the Parisian haute couture industry had been re-established. In Britain, garment manufacturers had adopted a pattern-making method from America to standardise garment sizing to allow them make completed garments for retail. Prior to this, garments were part-made by the manufacturers for completion in-store, within department stores in particular, to fit the wearer. In order to refine the system for mass-production purposes, given that British and American body shapes and sizes differed significantly, the British Sizing Survey of 1951 was conducted which aided the UK ready-to-wear industry 
in producing garments of a more-acceptable fit, thereby following similar sizing initiatives that had been undertaken in America approximately a decade earlier [21]. Couture gained its lead again as the major source of inspiration and as the main director of fashion for the mass-production garmentmanufacturing industry. During the 1950s and into the 1960s, in support of the man-made fibre sector and its new technical developments, fabrics and colour became more important in the seasonal direction of fashion on the high street. This was very much supported by the increasing numbers of trade fairs and exhibitions, such as Interstoff in 1959, that displayed fabrics as a source of colour direction. Interstoff developed as a trend-setting trade fair that is said to have conducted its own colour and trend forecasting activities, originally in Frankfurt, and later in the latter part of the twentieth century in mainland China and Hong Kong through the Interstoff Asia Division [22].

The younger generations also began to re-affirm their importance as directors of fashion through a newfound freedom of self-expression and the spending power to sustain it [23]. By the end of the 1950s the popular music scene further established itself as a driving force of fashion. As lifestyles became even more diverse than those of previous decades, more forecasting agencies were established, some of which specialised in particular areas such as women's wear, men's wear or children's wear. The forecast packages included style trends as well as colour trends. Other agencies found it profitable to focus on supporting the now firmly-established synthetic fibre industry. In addition, other countries established their involvement in colour forecasting. Donzé [24] notes that colour forecasting began in Japan during the second half of the twentieth century as its fashion and apparel industry adopted more western design and manufacturing processes. Scandinavian countries appear to have developed their interests in colour forecasting slightly earlier, as Giertz-Mårtenson [25] reports for example on the Swedish Shoe Fashion Council who played a critical role in their forecasting sector in the mid-1940's. More countries with a developed or developing textile and fashion / apparel industry would soon be involved in colour forecasting, either through their own trade organisations or through their engagement with larger bodies, such as the Color Marketing Group.

\subsubsection{The Color Marketing Group}

In 1962 the Color Marketing Group (CMG) was established in Alexandria, Virginia, as a non-profit-making organisation. Whereas TCCA (which then became CAUS) had been established to serve the American industry, CMG operated on an international level in more than 20 countries. Their main industry focus was on textiles in apparel and home furnishings and the organisation remains an effective colourforecasting support organisation to this day with around 1000 CMG members from around the world. Similar to CAUS, CMG produce biannual colour forecasts, though approximately 19 months ahead of a selling season, as opposed to two years ahead. Two international conferences are held where members identify and discuss relevant current colour trends and how these are seen to be changing. Other influential changes such as those taking place within societies, cultures, technologies, economies and politics are also discussed, with particular reference to different demographic groups. Steering committees then establish the colour forecasts to be made available to its members [26]. As more forecasting companies began to be established from the 1960s onwards many of these set out to develop niche forecasting businesses. For example, in the 1970 s there was a noticeable increase in forecasters establishing companies for the home furnishings and interiors sector; also as sportswear 
gained in importance in the 1980s, many forecasting companies were established to serve this particular industry sector [27].

\subsection{The importance of forecasting by the late twentieth century}

There has always been a degree of vulnerability for fashion and textile companies as their products must reflect consumer taste. Harsh lessons were learned in relation to style in the mid-twentieth century at a time when consumerism had developed in earnest and style rivalry was still more prevalent. Fashion disasters of the late 1960s and early 1970s instigated changes to the textile and fashion industries with serious financial losses and business closures, for example, the midi skirt versus the mini skirt. As Chrisman-Campbell [28] reports, the midi 'heralded a political and aesthetic revolution in womenswear, and a turning point in American consumer culture' and had a similar effect in the UK and around Europe. The mini had spread in 1964 across the Atlantic from London night clubs and was not expected to outlast the summer, yet cold weather was endured by women by wearing high boots and thick tights, and the mini hemline continued to rise until 1967 when it was so short 'it had nowhere else to go but down'. Chrisman-Campbell continues to recount that Diana Vreeland, editor of Vogue, attempted to make it clear to readers that the short style was not suitable for all women, but this failed to convince readers. However, the knee-length midi did emerge and by 1970 the mini was no longer seen in fashion magazines; but consumers were not ready to abandon it, though by now the fashion industry 'had too much invested' in the midi, and 'with inflation on the rise, the midi was [considered to be] an economic encumbrance [as] the longer length required a higher price point'. Newsweek reported on the 'warring interests of consumers, retailers and the fashion press' and Women's Wear Daily published a letter from a retailer who had complained that manufacturers and retailers were doing a disservice to consumers 'by trying to promote a fashion that [they] were not ready for.' Chrisman-Campbell quotes Vogue as having lost 38\% in advertisement revenue at this time and ultimately, the New York Times disclosed in August 1974 that 'women stayed away in droves, forcing several couture houses and small manufacturers into bankruptcy and the apparel industry into a tailspin'. Furthermore, many retailers were reportedly 'chopping off their unsold midis and marketing them as minis'. While such reports have not come to light as yet within the context of colour, it is accepted that colour can be a powerful sales tool, but only if the colours made available achieve consumer acceptance.

In the 1960's and 1970's, the forecasting sector offered new hope, not just for manufacturers and retailers, but for new fashion-related employment opportunities, as the more important forecasting agencies such as Informa Inc., Promostyl and the International Colour Association (ICA) were established. These companies in particular attempted to reduce the trend-prediction timescale for colour forecasting to around 18 months ahead of the selling season in order to be more responsive to consumer demand, and provide better predictions for the industry. Additionally, more of the forecasting agencies combined colour forecasts with style and fabric directions (fashion forecasts) to appeal to their clientele. Designers and manufacturers were finding it increasingly more difficult to dictate fashion and colour trends to consumers, as had been the case in earlier decades, and a more accurate method of colour and trend forecasting was considered to be the solution. Yet, as King [29] discovered by the time of her writing in 2011, there is a lack of inquiry into the accuracy of colour forecasts in academic literature which is possibly, and most likely due to the question of 'what parameters may be used to 
measure' the accuracy. King reports that forecasters and forecast information users (designers, buyers and merchandisers) often 'verify' their own colour forecasts against those of other forecasters and at trade shows, whereas retailers tend to use sales figures as their post hoc measure. These methods still do not guarantee that the colours forecast for a given season will be acceptable to consumers, especially if the measures are made post-production and therefore the issue with accuracy in colour forecasts identified as being problematic in the mid-twentieth century still remains unresolved. King proposes that there may be some value in colour cycle data or knowledge (colour cycles are discussed in Section 7.4.1) though also notes that there is still insufficient research in this area to allow for a critical evaluation of this resource let alone assure of its potential benefits. A particularly interesting disclosure given by King is that while there appears to be a general acceptance that colour forecasts are around $80 \%$ accurate, commercial forecasters are known to 'update' their colour cards as the season approaches 'where at least $40 \%$ of the colours are changed'.

During the 1970s the number of couture houses reduced, as cheap mass-produced fashions were better suited to the economic downturn that affected the fashion industry globally. By the 1980s haute couture had lost some of its influence and importance in determining fashion direction, even though demand increased in the Middle East due to its successful oil industry [30]. Retailers in the USA, UK and much of Europe attempted to respond more to their own target market's needs by taking greater responsibility for identifying trends for themselves and by working more closely with forecasting agencies as they developed relationship-marketing strategies to increase brand loyalty. This may have instigated the creation of co-existent trends as experienced today [31] or even the diversification of trends that had already been felt within the industry and had spurred retailers to develop more-robust marketing strategies with the inclusion of trend forecasting.

\subsection{The role and importance of colour forecasting today}

Colour forecasting is reputed to be the backbone of the fashion industry (not least by colour forecasters themselves) [32], however, there is a dichotomy within the textiles and fashion industry as to its importance as some hold quite negative viewpoints [33]. Colour forecasting takes place at the very beginning of the process of deciding what should be manufactured; it forms a critical part of fashiontrend-forecasting where seasonal colour change is used to stimulate sales through planned obsolescence and in order to promote a sense of the new [34]. Forecasting is considered to be both an art and a science; an art because of the use of intuition, good judgement and creativity, aspects of the approach which are discussed in detail throughout Sections 2, 3, 4 and 5, and a science due to the analytical concepts and models used to predict trends systematically [35], which are discussed at length in Sections 6, 7 and 8 (more care should be taken in the use of the term science in this context. As Craik [36] identifies, the real challenge that fashion companies face is the accurate prediction of future trends; this was considered to be necessary due to the traditionally long lead times between the production and colouration of fibres, yarns and fabrics to the finished garments being available to purchase. However, as this timescale has reduced considerably more recently due to the streamlining of fashion supply chain management systems, the need for forecasting so far ahead of the retail season today may be put to question; a question that may be answered throughout this Textile Progress. Trend forecasting companies assist fashion businesses to understand colour trends through the provision of seasonal 
colour palettes that they predict will be popular for a coming season [37]. Trend colours are used by fibre and yarn manufacturers for the development of their own colour forecasts and shade cards, which are exhibited at trade fairs [38]. Fabric and knitwear companies create their own colour forecasts based on both fibre, and yarn company forecasts and those from the primary colour forecasting organisations [39]. For fashion companies, understanding future trends and their longevity is important as this enables them to develop marketable products; such understanding contributes to their ability to tailor their own marketing strategies in line with their target customers' needs and preferences [40].

Colour is a critical component of marketing due to its ability to affect our emotions [41] and colour has long been recognised as being a powerful tool for the sale of consumer products and their positioning in the marketplace, because of its influence on consumer purchasing decisions [42]. In addition, brand loyalty has been found to be enhanced when retailers more-frequently offer the colours that their target consumers feel suit them [43] and in particular, using consumer colour preferences correctly can vastly improve sales [44]. Today, forecasters use consumer research to identify changing patterns in lifestyles and preferences in order to anticipate future trends to inform product development, retail strategies and brand management [45]. Colour management strategies are used not only to attempt to satisfy consumers through colour choice, but also to communicate colour information through the design and production stages [46]. Subsequently, the colours a company selects for its product line season-by-season connects the company to the seasonal colour forecasts [47]. This colour selection process is considered critical as it will determine the success of sales through the acceptance of the colours as determined by consumers [48].

While forecasting is a complex, multi-faceted process [49], that involves the analysis and synthesis of information from many sources, both inside and outside of the fashion business, it also involves working with extensive data sets related to consumers. Currently this is mostly achieved through the analysis of past sales data [50]. While attempts are made to understand consumer preferences through a range of consumer-analysis techniques, which allow forecasters to examine patterns in consumer characteristics and behaviours [51], these attempts are applied largely to consumer products that have longer trend cycles than those of fashion apparel. Undertaking similar market research for fast moving trends is not feasible due to the speed of change that is being driven by the industry [52], an issue which is further explored in Sections 3.4 and 7.2. However, colour preference data is considered to be a critical element of the colour forecasting process in order to remain profitable in a competitive marketplace [53]. Such data is currently under-used due to a lack of sufficient investigation. To date, research into colour preference has followed in the footsteps of Hans Eysenck's [54] Significant Pattern of Colour Aesthetics study. Eysenck found a common order to colour preference which he described as universal; his findings place blue as the first colour preference followed by red, green, purple, yellow and orange [55]. Such studies simply rank a limited number of basic hues without any consideration of the subtle nuances of colour that is essential for colour forecasting. As a consequence, according to Whitfield and Whelton [56], colour and trend forecasting companies work on the assumption of a natural order to consumer colour preferences, which they believe is a reflection of the zeitgeist - zeitgeist being German for time (zeit) + spirit (geist) - though without any substantive evidence. Diamond and Diamond [57] recognise that predictions are not actually possible. In addition, the anticipation of consumer acceptance is a risky 
business strategy. Consumers are also now less willing, or at least appear so, to follow trends set by industry professionals, such as forecasters and designers. Diamond and Diamond [58] further state that due to consumers' ability to accept or reject fashion, though for what reason is not given, a greater awareness of consumer preferences is necessary. This is a notion shared with trend researcher Anne Marie Commandeur and one that will be revisited later in this article (see Section 9). Commandeur is the founding member of Stijlinstituut, Amsterdam, an agency that is marketed as a 'source of information for the style related industry, reporting on consumer mind-set and environment, technological developments and trend-setting movements' [59]. For Commandeur, the need for colour forecasting today relates very much to consumers, in particular to provide them with inspiration and confirmation of trends. She notes that many consumers are 'reluctant to make their own decisions or hesitate to choose something they haven't seen in fashion magazines or in the homes of their neighbours', as for many, what we choose to wear is determined by what other people wear. However, Commandeur reflects on this then from a commercial perspective, noting that the manner in which people in general are influenced by others is problematic for, perhaps the more innovative, niche market companies wishing to introduce a 'unique colour palette' in a marketplace where the industry targets the mass market [60], thus highlighting a negative viewpoint of the consequences of colour and trend forecasting. Throughout this Textile Progress the different perspectives and the repercussions of forecasting are evaluated and deliberated and a comprehensive understanding of the colour forecasting process, its role within the larger trend forecasting system and as a twenty-first century business strategy are determined.

\section{Developments in the art and science of colour forecasting}

Colour and trend forecasting are considered to be both an art and a science due to the range of subjective and objective tools and techniques used. The more-objective characteristics of the colour forecasting process and the objective tools and techniques themselves are analysed later (Sections 6, 7 and 8) whilst Section 9 explores the human-business interface.

\subsection{The art of colour and trend forecasting}

Linton [61] proposed forecasting to be akin to identifying and visualising images of the future. However, such an ability to recognise and imagine future products and scenarios is only a part of the forecasting process, rather than the perceived end result. Rousso [62] describes forecasting as 'the practice of predicting upcoming trends based on past and present style-related information'. To the novice this may suggest the practice of gazing into a crystal ball but on the grounds of having previous knowledge of what has been popular, or not, in the past and using up-to-date knowledge of what is currently available to consumers, and perhaps what they are wearing at this time. It is the term prediction that evokes a sense of mystic activity by a chosen few and for some instils conviction of a confidence trick. Even Scully and Cobb's [63] expression of 'predicting probable colour and trend directions' leaves doubt in the mind of the would-be trend information user of the accuracy, or even just the likelihood of the forecast information having any real relationship to future actuality in the context of fashion. The mind is not put at ease as they continue with 'even when a colour palette has been carefully developed and is on-trend, it may simply fail to resonate with the wants and needs of a particular customer at that moment in 
time'. Yet companies are prepared to pay a significant amount of money per year to reduce the risk of getting their product offering wrong. Even though some of the most influential forecasting companies openly state that what they are providing is merely a resource for inspiration, such as Worth, formerly of Worth Global Style Network (WGSN), which had established in 1998 in London [64]. Worth sold the forecasting company to the British Media Company eMap in 2005 for $£ 140$ million coolly claiming that they did not predict or forecast, they 'provide inspiration for creatives to create trends' [65]. For the forecasters who defend their honour their methods are a mix of the subjective and objective, the qualitative and the quantitative supported by a mixed bag of skills and knowledge that enable them to promote and sell their trend information with confidence. The approach to the process is therefore both an art-form and a science. For the sake of the critics, the more grounded the analytical process and the more the data is based on hard facts then the more convincing the outcome may be. Aforementioned trend researcher Anne Marie Commandeur believes that the word forecasting is problematic in itself as it 'evokes an image of sorcerers with a crystal ball, but there is no magic involved, only inspiration and clear thinking' [66].

\subsection{The objective methods of colour and trend forecasting}

As stated above, the soft methods, tools and techniques of colour and trend forecasting constitute the art of forecasting. In general authors tend to focus on these methods, particularly in fashion textbooks, as they have a certain allure and support the mysticism of prediction. For the sceptics the notion of unreliability of the forecasts and the concept of it all being arbitrary hocus pocus is reinforced if only soft approaches are applied. Application of the more-objective methods (or otherwise loosely-termed science-based methods) can typically however, demonstrate a bona-fide process of data collection combined with analysis by well-respected methods, tools and techniques (as is the case with the social sciences). These are methods that could be repeated by others, which is not the case with the soft tools and approaches. As with the social sciences, the results themselves however will be subject to change. The objective methods may be considered to be far less attractive to the average fashion reader and also rather tedious to write about, yet, as the trend forecasting sector has developed, these 'hard' approaches to forecasting have become far more important. As more companies compete there is a stronger need for forecasters to prove their worth and to be better able to deliver quantifiable prediction information, though still with creative flair. The objective methods, tools and techniques, as we shall see in subsequent Sections, are grounded in theory. However, we are still largely dealing with the measurement of subjective nuances of human nature and behaviour; as Raymond [67] reminds us, a trend is the movement of 'something ... which tends to move ... which has a consequential impact on the culture, society or [the] business sector through which it moves', where the direction of the movement becomes one of the tangible measurements of the trend. Raymond also notes that 'trends are a fundamental part of our emotional, physical, psychological landscape', and hence 'by detecting, mapping and using them to anticipate what is new and next in the world we live in, we are contributing in no small way to better understand the underlying ideas and principles that drive and motivate us as people'. Kim, Fiore \& Kim [68] state that the movement of a trend 'mirrors change in social, cultural, economic and technological factors within a society'. It is therefore implied that understanding trends has far more value than just in the commercial sense, that good forecasters, or good forecasts can 
enable a far greater understanding of the human race, at least in relation to what we do, how we do it, and maybe even why we do it, depending on the effectiveness of the objective methods and tools used for the analysis of the identified trends. In relation to colour forecasting and trend prediction for consumer products, or fashion(able) products, McKelvey \& Munslow [69] identified a change in the way that trend forecasting is approached now compared with the earlier decades of the twentieth century when forecasting was coming into being, namely that before the 1960's single dominate trends were evident, whereas since the 1960's trends have become 'more pluralistic'. They rationalise this as following 'the expansion of mass communication and in turn the increasing sophistication of the consumer', though this would appear to be a very simplistic analysis of many factors that would have contributed to the changing ways of trend forecasting. Competition from the growing number of forecasters should not be disregarded, as well as all of the other macro-environmental factors, such as socio-economic, political and cultural influences.

As the forecasting sector grew over time and forecasts were developed for different purposes and for different audiences, the colour forecasting process undertaken by leaders in the sector such as CAUS and CMG began to differ from the process undertaken by fashion forecasters of smaller agencies (and also by designers who also engage in a forecasting process). Rousso [70] suggests that the colourforecasting process is more simplistic than the fashion-forecasting process as the focus is more on identifying colour shifts. In any case, each forecaster appears to develop their own way of approaching the forecasting process, be it for colour or for more general fashion forecasts, and authors who have written about forecasting methods also differ; such variations are examined in Sections 3, 4, and 5.

\section{Improvements in the colour forecasting process}

While many forecasters would like the public to believe that their approach to forecasting is entirely intuitive, a clear set of steps, albeit somewhat iterative, can be identified. The basic process stages were outlined by Diamond and Diamond [71] and previously by Healey [72-77] though not in any great detail. Irene Zessler [78] gave a very brief and, what is considered by this author to be, a sketchy account of how the Peclers Paris colour range is compiled. Zessler designed the fabric collections and consequently became involved in the colour-forecasting process; she states that at that time, Peclers created its own colour range for internal use which then informed the forecasts they developed for their clients. This was conducted independently of Premiere Vision (PV) but coincided with the timing of the trade fair. The colour range was developed specifically for fashion fabrics working with all areas of the textile industry, such as spinning and weaving. The format of the forecast information was very particular having a set number of colours and colour combinations, as well as a set number of photographs to support the colour choices. The colour data was then put into context for the season they were working on. Zessler worked two years ahead of the selling season and claimed to always work with the end consumer in mind. She collected colour representative samples, mostly in textile form, such as fabrics, ribbons and trims. She attended brainstorming sessions in the office at Peclers headquarters bringing together sets of forecast inspirations, activity which is professed to be undertaken with awareness of cultural and social trends. The remainder of her chapter focuses on examples of colour representations and art supplies used for developing the colour ranges, such as artists' paints and fragments of fabric. 
Brannon [79] put forward a step-by-step method for colour forecasting. The stages given in the model are systematic with no suggestion of any iteration. Later, Brannon \& Divita [80] proposed that the forecast itself should 'identify the source, [the] fundamental mechanism, [the] direction and tempo of the trend. It is assumed that the tempo refers to the pace of the trend, or perhaps more specifically, the speed of the trend in relation to how quickly it will be adopted by the general public, or target market. The direction of change and the speed of change are expanded upon in Sections 3.4 and 7.2. Brannon's six steps for the development of a colour trend forecast are as follows, along with a critical analysis of each:

\section{Brannon's Six Steps}

1. 'Identify the basic facts about past trends and forecasts'

Exactly what those facts are is left to the reader's imagination, the precise nature of the colours (colour measurements), the markets and products perhaps where they were favoured, and for how long for, can be assumed. The type of data, being factual, would suggest that this early stage involves a moreobjective than intuitive approach to the development of the forecast.

2. 'Determine the causes of past change'

This would again suggest that the more-rational methods of data collection and critical analysis are necessary (rather than the priority being given to intuition and emeotion). The depth and breadth of data collection and analysis this stage would be at the discretion of the forecaster. The breadth may include an exploration of drivers such as social, economic and political factors and other effects such as celebrity influences and new technologies. The timescale to produce the forecast would have a bearing on the rigour and quality of this stage.

3. 'Determine the differences between past forecasts and actual behaviour' Essentially this would suggest that there should be some investigation into the accuracy of previous forecasts. It is not clear whether Brannon is discussing the forecasting output from a colour forecaster working for agencies such as CAUS and CMG, or referring more to trend forecasters working for the smaller niche-led consultancies, or even to designers who create their own trend forecasts either prior to the design process or as part of their design process. The differences are worthy of some consideration. Colour forecasters such as CAUS develop their forecasts around two years ahead of the relative retail season and therefore the forecasts are not as responsive to factors that would be identifiable through Steps 1 and 2 described above. Also, the forecasts from agencies such as CAUS are intended to be more wide-ranging in order to appeal to a variety of industries from which other (morespecialist) forecasters can take inspiration to develop their own forecasts for particular products and target markets.

4. 'Apply forecasting tools and techniques, paying attention to issues of accuracy and reliability' This title would suggest that Step 4 is the point where the art of forecasting begins. The tools and techniques are not defined in either of Brannon's publications, nor is there any in-depth discussion of how accuracy and reliability of the forecasts can be addressed.

5. 'Follow the forecasting continually to determine the reasons for significant deviations from expectations' 
It would appear that, for the forecaster, there should be some kind of monitoring of a forecast in order to observe its trajectory. It is assumed that to know why the forecast has deviated is to be able to rationalise why the forecast has not come to fruition (if asked). This step is likely to be executed using one of the methods described in Section 7.

6. 'Revise the forecast when necessary'

When to undertake this final stage would be dependent on the timescale of delivering the forecast to its audience. To revise the forecast once available to the client would not be easy unless, as a forecaster, you are providing your customers with regular updates on how the present forecast is moving. However, in order to do this with any real degree of accuracy would surely require the colours of the trend to be available to the general public in order to observe the popularity of the colour(s). Monitoring the trend at this stage suggests a move back to step one where the process would begin again for the next forecast.

What can be taken from Brannon's Six-Step Process is that forecasting itself is cyclical as demonstrated by the need to revise forecasts before beginning the process again for subsequent forecasts and that developing and monitoring colour forecasts would require the attention of a dedicated professional team. For fashion designers using the output from fashion forecasting in their design and productdevelopment work, the post hoc monitoring of the colour forecast in terms of its success as a sales tool can be easily quantified through sales volume. Marked-down stock on the shop floor may also be used as an indication of weakness in adoption of the colours from a forecast. Mistakes in colour choice at the design stage can be seriously costly to the retailer, and therefore colour is considered to be a powerful sales tool [81] (also see Section 6.5), not only to encourage sales but also from which to monitor consumer moods and behaviours, or indeed to influence them. Brannon had recognised the need for all forecasters to be sensitive to changes in consumer habits and preferences and to use these to inform the trend forecasting process.

Brannon and Divita [83] expand on Brannon's previous six-step approach to give a seven-step process for the development of a forecast. While Brannon and Divita do not clarify why this additional step is included we may presume that it is a consequence of the need for the trend-forecasting process to evolve, either to produce more accurate forecasts per se, or for forecasters to at least claim a more rigorous process. Step 4, the new inclusion, is: 'to determine the factors likely to affect trends in the future'; the rest of the steps, renumbered to allow for the new Step 4, remain as they were. The newlyinserted Step 4 would suggest that part way through the forecasting process some form of scenario analysis is undertaken, though how the forecaster would use this knowledge to inform the remainder of the process is not given.

Rousso [82] also presents a method for forecast development though having only five distinctive processes, or stages as follows, the titles of which have been created for the purpose of this Textile Progress:

\section{Rousso's Five Steps}

1. The Research Process: 
First there is the research process to gather images and information to be used in the second step. This first stage stipulates that the researcher should be looking for novel inspirations and also acknowledges that both past and current trends should at least be observed.

2. The Data Sorting Process:

Through the second stage of the process, the data collected is sorted using an editing method where the fundamental objective is to identify patterns in the collection. One assumes that the items that should be discarded are those that do not relate to the themes identifiable through the existence of common factors.

3. Analysis and Interpretation:

The analytical part of the step should be strongly linked to the editing conducted in Step 2, otherwise the relationships between data may only be tenuous or even presumed. In Step 3 the 'causes' are to be identified, though it is not clear what causes are being referred to as there is little suggestion in the first two process stages that previous trends are under scrutiny; rather there is a suggestion that the researcher is sourcing data that promotes the concept of newness for a trend. Step 3 does however propose the expectation that newly-developing trends are explored together with the reasons behind those trends with a view as to how those trends will materialise. This would suggest that Rousso is referring to consumer-behaviour trends that are organic as opposed to marketed trends which are more product-specific, such as colour.

4. The Prediction Process:

Prediction is achieved by the forecaster after working with different scenario settings 'to foretell projected possibilities', a description with the potential to lead a sceptic to deduce the presence of prophesy or even guesswork as opposed to bringing into play data analysis that would, to the best of its ability, reduce the risk of misreading the needs or preferences of consumers.

5. The Communication Process:

The communication process (if indeed this step can be considered different from the previous one) is where the forecaster, according to Rousso, expresses 'thoughts, opinions and predictions' through presentations, mood boards and text.

Such descriptions of forecast development retain the potential for critics to consider that the forecasts themselves are merely the fantasies of a vivid imagination. Rousso may however redeem herself in the eyes of some in that she recognises that forecasting is not a simple process and that a great deal of skill is required to produce the kind of valuable forecasts that result from the application of subjective tools and techniques mixed together with objective methods, as discussed in Sections 7 and 8.

In addition to her five-step forecasting process, Rousso [84] also devised three questions that should be asked, the answers to which are to be found when engaging with the forecasting process:

i) What has already happened in the past that influences fashion today?

ii) What is presently happening that will notably affect fashion in the near future?

iii) What is likely to occur in fashion in the distant future?

The first question is akin to Brannon and Divita's (2015) Steps 1 and 2 with the additional reflection on the current time. Question ii) is loosely akin to Brannon and Divita's Step 3 and Rousso's final question 
links to Brannon and Divita's Step 4. This would all suggest that there is a significant analytical process that should be, or might usually be undertaken prior to engaging with the creative part of the process, in other words, working with inspirational materials and developing the actual forecast that will be presented to, and be used by the intended audience.

Fiore and Kimle [85] also gave a method for the forecasting process. Their approach was from the designer's perspective. The method has two main objectives, the first is to understand the evolutionary nature of colour (and of other design aesthetic attributes) and the second to 'predict future preferences' that are informed by the evolutionary nature of change in accordance with the social context. Fiore and Kimle's method begins with the collection of data and attention is given in its analysis to the frequency of occurrence, or patterns in the data. The data is only used as a source of inspiration because no particular reference is given to the analysis of previous trends.

Colour-forecasting 'guru' Li (Lidewij) Edelkoort describes the data-collection stage as being one of 'accumulation' from which a trend, or a concept is gradually formed, or visualised, and that it is the analysis of the concept that begins to provide the philosophy, or rationale for the trend. Edelkoort perceives this step to be of extreme importance along with the use of intuition. She declares that it is the questioning of the intuition that enables more depth to be given to the reasoning of the concept [86]. It could be said that this aspect of 'mysticism' somewhat disguises the analytical processes including those of thought and decision-making that eludes the novice forecaster. Edelkoort does however claim that the process is totally artistic with no reference to scientific methods, i.e. it is entirely intuitive, though many other forecasters refute this claim (see Sections 6, 7 and 8). It is interesting to note that despite Edelkoort's dismissive consideration of a scientific approach to colour forecasting, McKelvey and Munslow [87] describe her as the leader in the 'science' of trend forecasting, (maybe) due to her role as Directrice of Studio Edelkoort and founder of the internationally-renowned trend company Trend Union, which is based in Paris, New York and Tokyo [88], together with her membership of the steering committees for many of the large European Textile shows. What Edelkoort appears to be inferring is that science in itself plays no direct part in the process.

Colour consultant Julie Buddy who worked for the colour trend agency Informada in the 1990s identified three main stages of the forecasting process as being exploration, evaluation and analysis. If, indeed, Edelkoort questions her intuition, then we could presume that she does also consciously evaluate and analyse the concepts to some degree. At an event in Glasgow 1999 [89], Edelkoort gave the following brief account of her working method. She made reference to colour representatives, any substance having colour that could be used as a point of inspiration and reference, now or in future, are collected and stored. She explained how she has drawers full of 'bits and bobs' of the same or very similar colour which she revisits every time she is creating a new colour forecast. The selection process she declares is merely intuitive, appealing to the eye. The selected colour representatives are laid out on a surface to peruse and natural colour stories emerge from the array of colours. Ideas for the application of the colours, such as for fashion, emerge and story boards are then developed. The colour story boards are taken to the colour meetings described in Section 4.1. 
Even though Edelkoort believes that the colour-forecasting process cannot be described beyond her brief explanation, and cannot be quantified in a science-based methodology, the following subsections describe the stages of the art, or the practice of colour forecasting, and provide a model of the process before introducing some of the more theoretical concepts of trend forecasting and the evolution of trends in Section 7.

It is prudent also at this point to consider fashion-forecasting's place in the fashion-design process. Like the colour-forecasting process, the fashion-design process is also cyclical and comprises a range of identifiable and iterative activities that occur during the research and experimental stages. Also similar to the colour-forecasting process, a number of design tools need to be used knowledgeably and intuitively to bring about a design response to a given brief. The design brief sets out the requirements for the end product, and once fully understood the designer uses mood boards (see Section 5.3.1) and sketchbooks to explore, experiment and record ideas and inspirations as they emerge from the research processes. A range of boards may be developed including colour, fabric, design or styling boards. Designers use a wide range of visual inspiration sources akin to the forecaster, in addition to market reports, information from forecast agencies and trade fairs and observations of people, celebrities, musicians and public figures. Designers use their knowledge of colour, texture and materials. They also draw on their knowledge of recent style and themes and of those that consumers have not seen for some time in other words, using their knowledge of fashion trends. Designers are also aware of the social and economic trends that influence the acceptance of particular styles and products on the high street, which is used to inform the initial research and visual data collection stages. Designers then develop their mood boards. The manner in which the visual representations are arranged and manipulated allows the designer to see new connections between the visual data sets. This process of exploration acts as a catalyst for the creation of design solutions as the designer makes meaningful connections; this is a highly-intuitive process. Exploration is followed by a stage of selection and elimination which leads to a more-purposeful research stage which is often repeated periodically until the designer is satisfied that the mood or theme is fully developed, meets the needs of the brief and provides sufficient inspiration for the subsequent design and product development stages. The positioning of the visual data is very important as the juxtapositions promote creative and innovative design decisions. The designer may continue to work through more specific product related design ideas using a sketchbook or through further boards such as colour story boards, fabric and design/ style boards [90].

Designers work with the design elements and principles of design. The design elements are generally considered to include space, line, shape or form, colour, texture, pattern and light, each of which stimulates emotions which are further determined through the manipulation of these variables known as the principles of design. Designers work with the design elements initially through specific boards such as colour story boards, fabric and style boards and through sketchbooks; they may choose to work solely by hand or on a computer or to combine the two methods. The principles of design in theory can be defined under one of three types;

i) directional - being the most simplistic,

ii) Highlighting, and 
iii) synthesising - which is the most complex.

However, most designers will work with the principles in a very intuitive manner rather than applying a more methodical process. Directional principles are used to lead the eye around the design; these include repetition, parallelism, sequence, alternation, gradation, transition and rhythm. The highlighting principles, emphasis and contrast, are used to bring attention to a particular point of interest. The synthesising principles are particularly important in fashion design as they do not simply create aesthetic appeal, they also make sense of the whole ensemble through relationships including proportion, scale, balance, harmony, and unity [91]. The synthesising principles in particular constitute much of the experimental work when considering garment style through working with the overall shape or silhouette, such considerations will affect and inform the pattern cutting stage later in the process. Many sketches are developed throughout the design process. Eventually a small number of designs will be selected for the prototyping and production stages of the fashion design process [92]. Figure 1 shows a simplified model of the fashion design process.

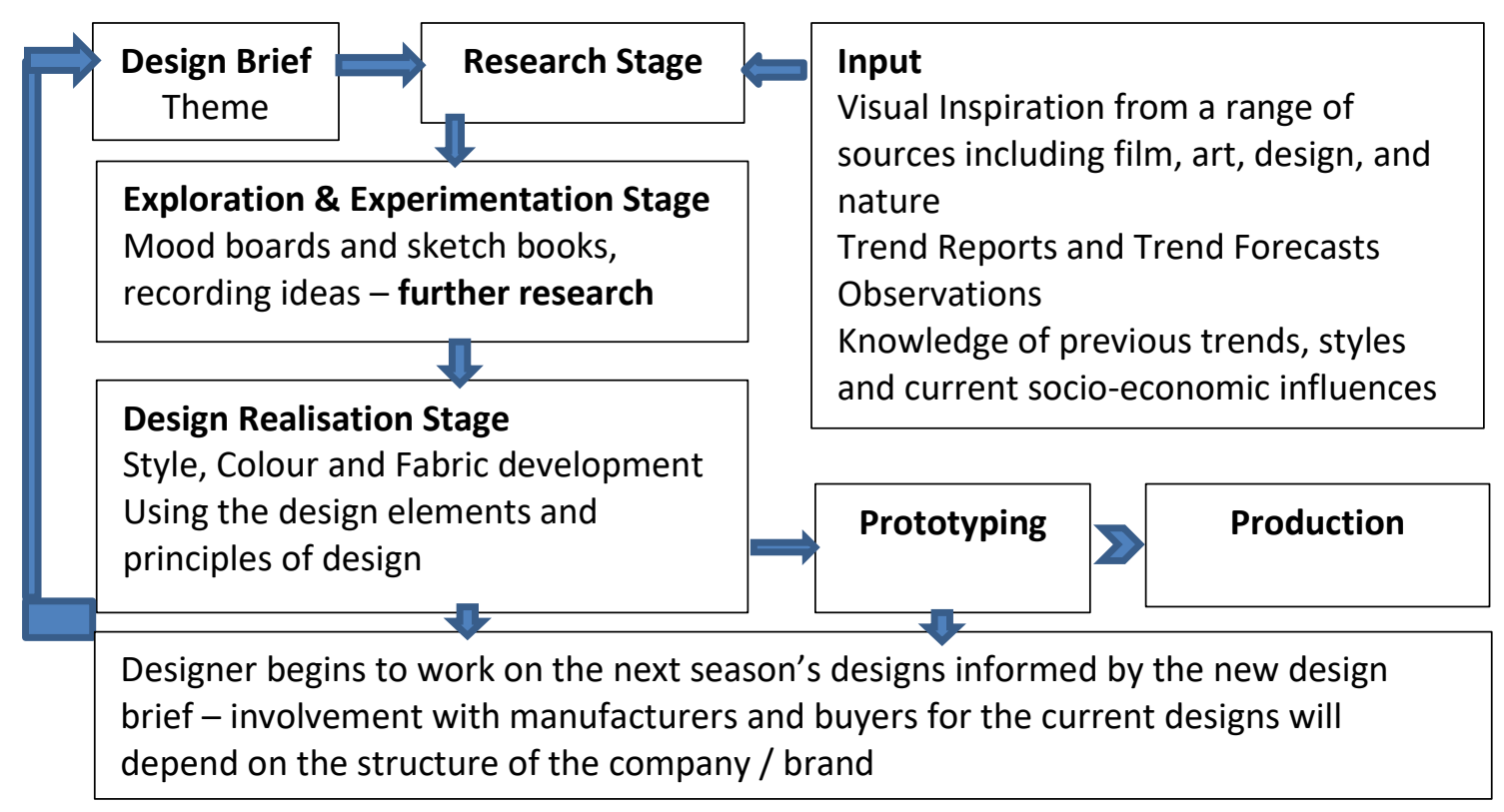


Figure 1. The fashion design process (Source: The author's own interpretation).

\subsection{Sourcing inspiration and data collection}

Colour inspiration can be taken from a great number of resources which themselves can be classified as being subjective or objective sources. The information obtained from subjective sources is often unrecorded and may not always be readily available. Subjective information is obtained through the soft skills, namely awareness, intuition and observation (see Section 5.2). Resources may include shopping trips, visits to galleries, museums and theatre, fairs, festivals, streets, clubs, pubs, parties and television. Wherever there is something of interest to observe and from which inspirations can be drawn. When observing people, moods can also become a source of inspiration. Observations may be retained in the memory but hard records are also obtained, through for example, note-taking, sketching and photography. Once recorded, the information allows for more-objective techniques to be applied and it becomes more available for others to use. The data may include previous trend information, magazines and newspapers, blogs, archives and other tactile items as representations of specific colours [93]. Designer collections and catwalks can also be used as a resource [94]. According to McKelvey and Munslow [95] entire colour palettes may be derived from, or inspired by, exhibitions and gallery visits, plus other similar places of cultural interest where historical or unusual imagery can be found, which does not suggest that a rigorous process has always ensued. However, in order to predict new trends, forecasters collect data to understand cultural and consumer shifts in how they live, think and behave. Forecasters often travel extensively to observe and record points of particular interest to them from the art-world, and also inspirations from fashion, music, and other factors where culture can influence fashion and changes within trends. They also undertake their own market research (see Section 8.4). Their primary objective is to identify changes in society, culture, the economy and technology.

\subsection{The colour-selection process}

According to Montgomery [96], colour selection begins 'first with [the] personal taste or subjective experience' of the forecaster. Colour selection for the commercial marketplace is largely determined by design teams and buyers, who understand their target consumers' preferences through previous sales. Colour palettes, or colour-ways are developed using colour forecasts [97]. According to McKelvey and Munslow [98] knowledge, through experience, and one's own judgement are essential in order to select the 'right' colour palette and colour stories; this is because of the importance of colour as a sales tool. As they also state, 'colour can make or break a season'. It is considered to be prudent to note here that colour representation on colour cards and small fabric samples may be poor in relation to the colour achieved when it is applied to its context, in a garment for example, and as stated by Tan [99], 'colour all by itself says nothing. It says something only when it is in context. Colour without product is also nothing'.

Therefore, translating colour from forecasts into product and product ranges may be where the colour forecasting system fails its users. Forecasters believe that those in the industry do not just want to be guided to the correct basic colour, the nuance of the colour also needs to be appropriate as does its 
communication as explained in Section 9.1. However, the choice of colour made by those in the industry has often been blamed for poor sales volume and consequently low revenue and profit. McKelvey and Munslow [100] for example state that retailers and brands have lost millions of pounds/dollars as a result of using the 'wrong colour palette'. To complicate things further, different markets respond differently to colour and brands often use colour stories as a way to build brand loyalty and a point of difference [101]. A colour palette is a range of colours. Colour palettes are developed from colour stories which may or may not be colour forecasts in themselves. The colours within the colour palette are used to sell the story through the way in which they are organised and ideas in the story can be connected and identified. A new colour story is created each season and more than one colour story may be developed. Colour forecasters often use a number of colour stories particularly when their clientele are very diverse. Colour forecasters provide industry, and consequently, consumers with colour choice through their forecasters according to Tan [102], because in her opinion the industry does not 'determine what colours people wear'. In order to create a colour story, the forecaster benefits greatly from a sound knowledge and understanding of colour theories and colour cycles as discussed in Sections 7.1 through to 7.3.1 and 7.4.1. Knowledge of colour psychology is also proposed to be useful, as stated by Dee and Taylor [103], because colour affects our emotions and therefore our behaviour, to effectively use colour it is necessary to understand its psychological impact, and possibly even its physiological effects on the human psyche [104]; the usefulness of colour psychology is explored in Section 9.2.

Tate [105] provides the notion of an approach for devising a colour story which is based on five distinctive colour categories. It is suggested that a colour story should ideally consist of eight to twelve colours comprising 'some fashion colours', some staple colours', 'warm colours', 'cool colours', 'neutrals and darks'. There is no rationale for this combination nor any evidence to suggest that it works. It should also be noted that the categories themselves do not allow for any particular colour to only fit into one category at any point in time. While there may be some general agreement that staple colours are white, black, grey, navy, some would categorise neutral colours, such as brown and beige as staple colours. For Rousso [106], khaki is also considered to be a staple colour, though some may class khaki as a fashion colour. For baby-wear, white, pink and blue may be considered to be staple colours. Fashion colours are very dependent upon the promoted trends. Tate's method may be construed as the designers' approach to colour-story development and does not take trend data into account, also, for example neutrals and darks (or shades) may fit into any of the other four categories that Tate provides.

While colour forecasters will invariably draw on the same inspirations season-by-season, and the social, cultural and economic trends will also be the same, individual forecasters will produce different forecasts, though with some commonalities. This is due to the creativity of the individual, their interpretations may differ which will affect the resulting colour palette. Also the imagery that is sourced will be different in some respects. It is also essential that colour forecasters view their forecasts from the consumer perspective, as the colour palettes should be appealing to them, and also bear in mind who their own consumers are (their clientele) maybe other trend and fashion forecasters, designers and product developers, or retailers [107]. In addition, the colour palette should be different from the previous season's colour palette, offering something new, fresh and exciting. It is also essential to have a 
wider historical knowledge of colour cycles (discussed further in Section 7.4.1) and the all-important zeitgeist explained in Section 3.5. Knowledge and research are therefore critical components of colourstory and colour-palette development. Research should comprise the current retail offering, what people on the street are wearing and what commonly is appearing in end-of-season sales, in addition to new ideas from designers' catwalks. The forecaster must also be aware and knowledgeable about the trajectories of current colours, in other words which colours are plateauing in terms of popularity, which are becoming obsolete and which colours are gaining favour; also whether there are any colours likely to have impact arising from activities in the film and media industries. As previously mentioned, knowledge of the evolution of colour preferences is also very important as radical changes rarely occur.

Rousso [108] describes how colour stories and colour palettes evolve from colour concepts, which are often quite broad-based, such as the concept of nostalgia for example, and from themes. Themes are smaller concepts where there has been some attempt to analyse the concept in order to bring about a focus. The concept of nostalgia may for example be reduced to the 1950s fairground or the flowerpower fashion of the 1960s-70s. One of the fundamental artistic approaches to theme creation and colour story development is narration. This is the telling of the story, or a story, based on the inspirations of the theme and its development. The narration communicates messages about the theme. It is descriptive, nonfictional, but informative. It provides essential details about the concept origins. It is supported by the research. In order to substantiate and place the theme into context market responses may also be weaved into the narration. Furthermore, Rousso notes that theme development is not totally an art because a scientific approach is undertaken to establish facts through research in order to develop a data set which includes 'tangible data from past trend information, historical records, new technology, existing objects and materials, books, magazines and current events, (such data is sought from) trade shows, manufacturers, retailers, and consumers [via] specialists and consultants'. Rousso speaks in depth about the development of themes. In general, a theme should be a dominant concept that is cohesive. In the context of forecasting she believes that forecasters 'identify the emerging forces in current society, understand what is fuelling the cultural shifts, consider the relevance of the changes, and communicate possible outcomes for the future through themes', therefore suggesting that themes should organically evolve from the social and environmental factors. This approach relates very strongly to the method used by more general trend forecasters and the major colour forecasting companies whose forecasting information targets a wide audience including trend forecasting consultancies. The inspirations are very much the same as those for any fashion forecast or design process which includes the media, entertainment and leisure, news, events and popular celebrity icons.

The method used for theme development for the forecast is also akin to the fashion design process (Figure 1) and the general forecasting process (Figure 2) following Rousso's [109] theme-developmentprocess description, a seven-step process that implies that this is the forerunner of the colourforecasting process, therefore suggesting that theme development is an independent exercise. The seven-step process is given with interjections of critical analysis and cross-reference analysis with Brannon's (2000) and Brannon and Divita's (2015) aforementioned forecasting processes:

\section{Rousso's Seven-Step Theme-Development Process}




\section{1. 'Research, artistic approach'}

This is the same as Rousso's forecasting method where a creative approach is used to uncover facts but also utilises the researcher's knowledge and memory, as well as their instincts and intuition. There is also the suggestion of undertaking an element of market research in order to explore peoples' attitudes and opinions and to conduct observations. All of the data is to be recorded. Formerly and consciously exploring the details and changes of past trends, as in Brannon (2000) and Brannon and Divita's (2015) methods would suggest that Rousso is reporting a more superficial approach to theme development as opposed to a rigorous in-depth enquiry into trends as would be expected of the forecasting method itself.

\section{2. 'Collect images to illustrate the theme'}

This suggests that there is a process of collecting images in a controlled manner having already developed or at least identified the theme. However, this appears to be contradicted in the following stage and deviates from her forecasting method that requires a sorting and editing stage prior to interpreting and analysing the data.

\section{3. 'Edit, interpret, analyse and predict the theme'}

It is interesting to note that it is now at this stage that the theme is predicted, which sounds as though instinct and / or intuition are at play, yet Rousso describes a process of identifying patterns in the data which would suggest a more-analytical approach; one of 'reviewing by organising, grouping or separating images and information to identify patterns'. The editing process allows the theme creator to discard data that is deemed to be unnecessary. Remaining data may be further categorised in terms of the degree of significance, or value, presumably in relation to the now predicted theme, and interestingly, as stated, the significance to consider the 'impact the theme will have on society'. In Rousso's forecasting method the editing process and the interpretation and analysis stages are separate, the theme development is given as one stage which may imply an iterative process. What is more interesting to note is that at this stage the theme creator is required to carefully examine the data in order to 'identify causes, key factors, and possible results, investigating what fuels upcoming trends, and consider why and how the trend will manifest'. This essentially places the first three stages of Brannon (2000) and Brannon and Divita's (2015) models into a more critical development stage rather than being used as a knowledge base on which to build the theme. The forecaster is expected at this point to explain how changes will impact on society through the theme which may provide an opportunity for some embellishment as 'reading between the lines and inferring significance' is sometimes required. The edit, interpret, analyse and predict process also involves a process of evaluation directed largely by 'intuition and decisiveness'. This process involves comparisons of data and a selection process that requires 'investigation and experimentation' to extrapolate the significance of the theme. At this stage the forecaster is planning future scenarios and solving any foreseen issues related to emerging consumer behaviour and consumer needs. For a step-by-step process this stage of the theme development is rather exhausting just to follow. The depth and rigor of all of these sub-processes at this stage must surely be lost on the new forecaster hoping to develop meaningful forecasts from theoretical accounts. The presentation of the theme becomes a critical aspect now as the forecaster provides a series of scenarios often in the form of visualisations. The preparation of the forecast as a deliverable is also included here in the form of presentation boards and the script.

\section{4. 'Create the title'}


The later stages of the process support the delivery of the theme to the intended audience. In terms of a process it may be more appropriate to bring Stages 4 to 7 together and to separate Stage 3 into its component parts in order to put more weight onto the most analytical and important stage. However, in Stage 4 a great deal of consideration is given to the theme and the most appropriate title which sends out a clear message about the 'spirit' of the theme.

\section{5. 'Develop a tagline'}

The concept may be further explained through a tagline, though why this would this be necessary if the theme is well expressed through imagery and the title is not really justified.

6. 'Identify the mood'

It seems rather odd now that the mood is identified at the end of the process and not in the earlier stages as this should have a bearing on the overall development of the theme. There is the expectation that the forecaster needs to give further clarification on how the theme is communicated as a representation of the intended emotional impact the message has, or should have through the presentation boards.

7. 'Write the story for the theme'

Finally, a narrative is developed to further explain the theme. This must be meticulously developed in order to clarify the messages of the theme.

Figure 2 below presents a model of the general forecasting process model combining Brannon's (2000) six-stage method, Rousso's (2012) five-step method, Brannon and Divita's (2015) seven-step method plus the descriptions provided by Julie Buddy and Li Edelkoort, as given in Section 3. Rousso's (2012) theme process, given above, is also mapped onto the figure in order to demonstrate how it can be used as a standalone process or as an integral part of the general forecasting process.

Research \& Data Collection Stage (Accumulation)

Informed by past and current trends, preceding predicted trends, consumer behaviour observations and reports, inspiration sources, current and future influencing factors Exploration and data sorting

Application of the forecasting tools and techniques

Building and writing the trend rationale

Evaluation, analysis, editing and interpretation of data

May incorporate further research

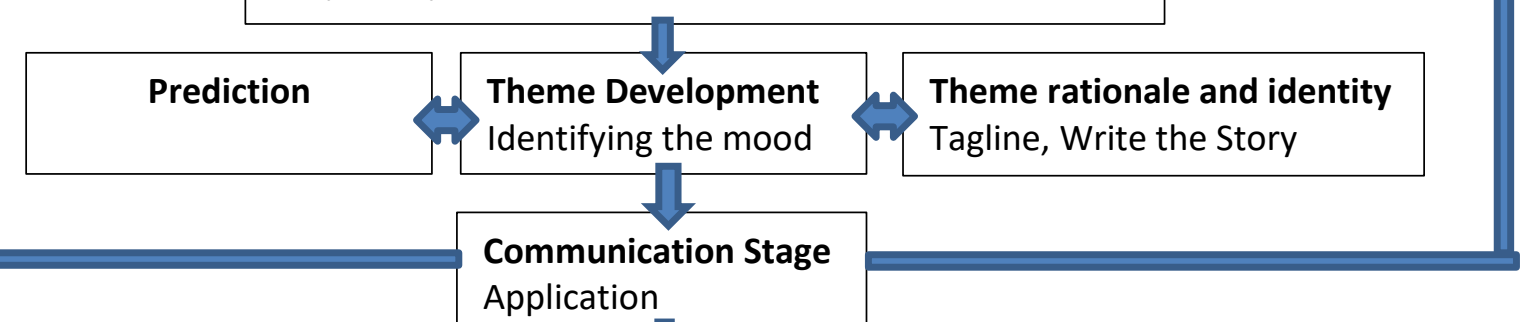

Tracking Stage (Optional)

Conducted simultaneously with the development of the next forecast.

Tracking the trend for validity and to refine the trend information reported regularly to clients / membership 
Figure 2. The forecasting process incorporating theme development (Source: The author's own interpretation).

Rousso goes on to further declare that, after all of the work undertaken to produce the theme, the actual colour forecast is developed. Whether or not Li Edelkoort does this as she works with her colour representatives to develop a new forecast is unknown, or at least undocumented. Edelkoort would have us believe that her process is much more simple and continual, often overlapping and iterative, which is perhaps why she does not attempt to describe it. Rousso [110] then provides a formal process solely for the purpose of the colour forecast. Whether or not this is actually a continuation of the theme development process that was critically analysed above, or can indeed be a standalone process is not stated, other than to say that 'following' the theme creation the colour forecast begins through taking the following five-steps, which are again critically analysed here:

\section{Rousso's Five Step Colour-Forecasting Process}

\section{1. 'Formulate the colour ideas'}

This stage involves research using both artistic and scientific methods. It is appreciated that colour evolves rather than radically changes season-by-season and therefore knowledge of past trends and current trends are required beforehand to then 'predict the future'. As a part of this stage the colour forecaster will visit relevant trade shows and fairs in pursuit of clues. This now seems to be independent of the theme that is suggested to have been created previously. The common sources of inspiration are given such as the media and catwalks, plus observations, political and economic reports and lifestyle trends. Online resources are mentioned also, such as blogs, which recognises the broader range of sources of present times due to communications technologies.

2. 'Develop a colour story' Here the connection is to Li Edelkoort's primary stage and the fourth stage of Brannon's (2000) model for this is the data collection stage where representative colours are brought together for the selection process to begin. Now the colour forecaster is reported to be working with the intended message of the theme previously developed. However, many colour forecasters use the theme development process as an integral part of the colour forecasting process, as discussed in Section 3.2, rather than as a separate process and certainly not as an add-on to the theme. Five to eight colours are professed to dominate the forecast with an additional number of colours which are incorporated to embellish or accentuate the colour story. This would then suggest that some of the colours included in the colour palette are not intended to be key colours of the forecast, though how an audience would differentiate these is certainly not clear.

3. 'Edit, interpret, analyse, predict the colour story' 
This stage is akin to stage three of Rousso's aforementioned theme development process with the exception of predicting the colour story as opposed to predicting the theme. In both cases prediction is a dubious term to use as previously discussed, but this stage also appears to confirm that Rousso disconnects these two processes where others see the two aspects as being very much connected and most likely informing each other. There is a clear advantage in connecting the theme development and colour story development processes to support the eventual communication and marketing of the colour forecasts, which may also be generally more time and cost effective. However, the codependency of these two processes may result in safe, contrived market-related forecasts from the onset. By separating the two processes and consequently focusing first of all on the theme development, more-creative and less-predictable colour forecasts with a fresh approach to the colour story develop for each forecast. The connection/disconnection of these two processes may indeed be the critical point of difference between high-end design, where a more innovative aesthetic is sought, and mainstream design, where brands seek to define themselves with some level of continuity for brand identity and brand loyalty considerations; it may also be indicative of the more highly-skilled forecaster, to eventually synthesise the theme(s) and colour story/stories in a creative and marketable manner, as a point of difference from, what we might call, mainstream forecasters where speed and commerciality are at the forefront of the overall colour forecasting process. Therefore, in the highly competitive environment, the disconnection of the two processes is more advantageous for product differentiation. Similarly, to the theme development patterns, which are to be identified in the data, for colour forecasting the patterns are to be found among colours that have been collected. It is made clear that the forecasters should be conscious of the decisions being made and the rationale for those choices. Closer examination is also required in order to refine through editing and to interpret meaning. It is necessary to marry the mood of the colours with the theme concept. Again this is suggesting that the process of theme development is conducted independently of the colour story or forecast which is then derived as a separate process but in relation to the theme. The logic only really makes sense if the forecaster has worked with the first three stages of Brannon (2000) or Brannon and Divita's (2015) models where substantial research into the social, cultural and economic drivers for example along with an interrogation of previous trends has occurred. The forecasts should be questioned also at this stage to appreciate the viewpoint of consumers to ensure one clear message is communicated to all.

According to Rousso, prediction then provides 'a recommended colour story that anticipates future evolution'. It would appear that Rousso perhaps uses the term prediction in relation to the scenario planning aspect rather than to suggest that the colour palettes are predicted. She does also make it clear that in this stage price points and target markets are an important consideration. This in itself would suggest that Rousso is possibly thinking more about the colour forecasting process as part of the product design process as opposed to the process that trend forecasters and colour forecasters of the major agencies such as CAUS and CMG would use.

\section{4. 'Assign colour names'}

In the fourth stage colour names are given to each colour preferably in relation to the mood. Colour names are also suggested to have universal appeal and to be linked somehow to colour perception and colour numbering systems such as Pantone. Here there is more suggestion that the colour forecasts are the intention of the colour forecaster or trend forecaster from the forecasting sector, as opposed to being forecasts developed by designers or product developers, as universal appeal for example would 
not necessarily be applicable to the company's target market if it is indeed the designer creating the forecast.

5. 'Write details about the colour story' In the final stage a summary is to be given of the concept and colour story details. This includes the 'evolution of the palette' and a 'prediction of the colour shifts', plus details of how the colours were chosen.

Diane and Cassidy [111] provide a visual representation of the colour forecasting process in a simplified form that allows the user to engage with each stage at any desired depth and using any number of resources for inspiration (Figure 3 below).

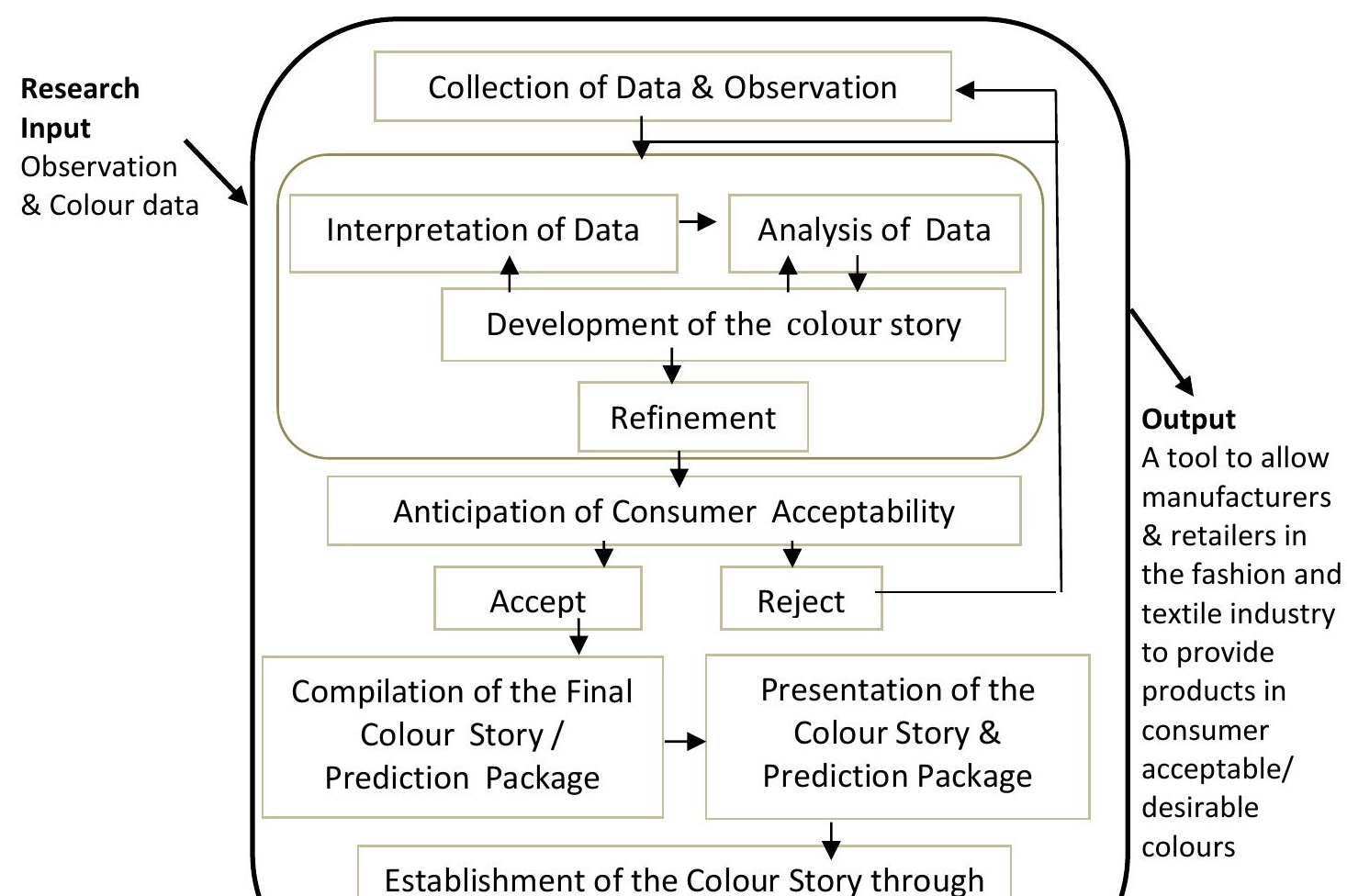


Figure 3. The colour forecasting process [111]

The input into the system can equally include all of Brannon and Divita's (2015) first four stages that closely examine present and previous trends and drivers such as societal changes, economic and environmental factors, colour evolution and colour cycles. The colour story and theme development are considered to be one and the same occuring through the interpretation, analysis, colour-story development and refinement stages. What is included in this model that appears to have eluded other authors is the anticipation of consumer acceptance. This may possibly be likened to Rousso's prediction, as there is no guarantee that the colour story will be favoured by the intended consumer, given that acceptance can only be measured after the products have been made available to consumers when retailers are then able to use sales data to monitor the success of the colour choices made by the designers and buying teams. However, sales data can be misleading as only the colours available at any one time can be monitored and therefore the knowledge of what may have sold better can never be realised. For the forecasters who operate outside of a manufacturing or retail company, the success of the colour forecasts will be observed and monitored in the next cycle of the forecasting process through observations of what people are wearing and what remains unsold in end-of-season sales on the high street.

Designers not only work with colour stories but also with colour-ways. Colour-ways give consumers a choice of colour within one product line. For example, a sweater of a certain style may be offered in two or more different colours. This strategy allows consumers to select a colour that they may prefer over the other colours on offer, or to enable them to better mix and match with other new garments or with existing garments in their wardrobe. For retailers, more product can be offered with no additional design input. Diane and Cassidy's model reflects the colour forecasters' approach with the prediction package being the end product of the process and the designers' approach to colour forecasting with the colour story being the end product of the process. In each case the colour-forecasting process as a system meets the same end in enabling fashion and textile companies to produce goods in colours that are acceptable or desired by consumers.

\subsection{Forecast types}

Sproles [112] proposed two forecasting principles, namely the general and the fashion specific. The general methods are those that use the scientific, or objective methods discussed in Sections 7 and 8 . The fashion-specific methods require research into social, cultural, and economic drivers plus technical developments and knowledge of the demographics of the intended audience or target market. It may be 
presumed that Sproles believed that the forecaster might engage with the general methods at some point, possibly in the manner that Brannon (2000) and Brannon and Divita (2015) prescribe. This is likely as Sproles (1979) also acknowledged that changes in economies, technical developments and lifestyles will impact on consumer demand. Sproles and Burns [113] put value on the different aspects of these principles. For example, trends in demographics were considered to have little importance to the colour forecaster who serves a wider market for their prediction packages, such as CAUS and CMG. Changes in demographics in relation to a market segment that the designer-forecaster compiles colour forecasts for, however, will be pertinent as this will have a bearing on the final products produced. The fashionspecific methods are proposed to encompass both science-based and artistic approaches by Sproles and Burns (1994). However, they would appear to suggest that the use of historical data and colour / fashion cycles while important are possibly less acceptable methods of forecasting for colour and fashion. It may indeed be that Sproles and Burns are suggesting that the science-based general methods should not be used independently or solely for the purpose of colour and fashion forecasts. In addition, there may be an assumption that the time involved in analysing colour cycles in a systematic manner may be too great to be useful to the process as the forecaster needs to move swiftly from one season's forecasts to the next. It is also considered to be extensive research that companies would be less willing to invest in but does leave opportunities for academics to investigate the theories and the scope of its role in the forecasting process. Earlier, Sproles (1979) had suggested that historic research into the last ten years of colour cycles and trends could be of particular value to assist in the process of forecasting for near future trend purposes. However, fads (see Section 7.4.2) for certain colours at certain periods of time would not be so easily anticipated. Further forecasting methods and tools include the measurement of diffusion, diffusion curves, rate of acceptance and speed of acceptance, these are aspects discussed in Section 7.2 and 7.2.1. In terms of acceptance and speed of acceptance to change however, for some forecasters observation and intuition is still very much favoured. This is discussed further in Section 5.2.

Another consideration is the time in advance of the proposed forecasts that the forecaster is working on, be it a short-range forecast or a long-range forecast. Not only is the time factor a prominent difference between the two types but also the potential accuracy of the forecasts. Short-range forecasts are considered to be more accurate as there is less likelihood of radical changes that could impact on the forecast. Short-range forecasts may be a matter of a few months ahead to around two years ahead [114]. The major colour agencies, CAUS and CMG for example, produce forecasts for around two years ahead of the retail season whereas designers employing the forecasting method may be working at around six months ahead. As the lead times in the manufacturing sector have reduced in more recent times [115] some designers may be working much closer to the retail season. Short-range forecasts better serve product development through the use of the design elements, including colour, as colour and style essentially evolve.

Long-range forecasts are generally three to five years ahead and therefore there is a higher risk of extreme and sudden change in events that may impact on the forecast [116]. Forecasting specialists such as The Future Laboratory (see Section 8), uses long-range forecasts and work on the assumption that changes are usually gradual and therefore reasonably predictable. For colour and fashion product trends, however, long-range forecasting would be too risky and quite possibly unnecessary. Long-range 
forecasts are however more suited to marketing and business strategies [117]. Long-range forecasting allows for more possible future scenarios to be designed and explored. This can be particularly useful for organisations when planning or re-planning their development and direction. The process involves scanning the environment, interviewing people and observations. The data becomes more meaningful through the analysis stage. Brannon and Divita [118] provide four considerations for long-range forecasting as follows:

i) The time frame is a minimum of five years ahead

ii) The techniques used should enable the forecaster to 'capture all of the signals'

iii) The methods used should enable the forecaster to correctly interpret those signals

iv) The forecasts should be focused towards a specific industry or industries or to a particular set of people

Long-range forecasts and short-range forecasts can also work together. The cultural shifts that are an important part of long-range forecasts are largely representative, or at least contribute to the spirit of the times [119] or the zeitgeist (see Section 3.5). Such cultural shifts are also an important aspect of short-range forecasting, in particular knowing at what stage the shift is in or likely to be in by the time the short-range forecast will be apparent in the public domain. It may be implied that periodic monitoring of the long-term should also be included in short-range forecasting, and that insights derived from long-range forecasts may aid an understanding for evaluating short-range forecasts [120]. Due to the scenario planning techniques used in long-range forecasts, the positives and the negatives are often identified, both of which are useful for informing short-range forecasts. Long-range forecasting can also benefit the fashion industry when taking materials into consideration as well as the design, manufacturing and retail strategies. Long-range forecasts may also benefit a company when attempting to re-position product lines, to extend product lines and for branding / re-branding purposes [121].

By using a blend of qualitative and quantitative methods, better quality forecasts may result [122]. The quantitative methods employed are those that relate to sales figures and volume analysis and to consumer behaviour analysis [123] whereas the qualitative methods are more concerned with the quality of the forecasts and are embedded into observation methods used by forecasters. Furthermore, Brannon [124] proposed two types of problem that the forecaster may encounter, 'wicked' and 'tame'. By definition, a wicked problem is 'a problem that is difficult or impossible to solve because of incomplete, contradictory, and changing requirements that are often difficult to recognize' [125], in other words, a problem 'for which there is no known algorithm to solve it. Examples include strategic planning, satisfying customers, transforming organizations, or protecting the environment', whereas tame problems 'can be solved by choosing and applying the correct algorithm. For instance, [where] multiplying the ingredients [or] changing the logistics' solves the problem [126]. Wicked problems are therefore more complex than tame problems. Tame problems are approached from within the parameters of traditions, beliefs and rules. A six stage process was given by Brannon to solve tame problems, the first and second steps are defining the problem and creating alternative solutions respectively. Stages three and four involve criteria, firstly to specify the criteria which is to be used to evaluate the solutions derived in stage two, and then to apply the criteria to those solutions with a view to determining the most appropriate one. Stage five involves implementing this solution, and stage six 
involves collecting feedback on the effectiveness of the solution. This process can be easily related to the forecasting process used by designers and forecasters operating at a consultancy level. The problem defined in stage one would normally be called the brief. The alternative solutions would be the generation of different colour stories for colour forecasting and different colour ways for the designer and product developer. The implementation of the solution would be the delivery of the product or forecast. How much of an evaluation process would be applied post-delivery would largely be dependent upon the diligence of the designer-forecaster.

Brannon's wicked problems are more akin to the forecasting process undertaken by agencies such as CAUS or more general trend forecasters such as The Future Laboratory, the kind of consultancy that uses trend data to consult on brand and business strategies as opposed to products. Wicked problems are said to require so-called 'out-of-the-box thinking'. Images and text are used but not as points of inspiration for products, also cultural references and metaphors are used to develop concepts related more to changes in societies and more global-reaching shifts in cultures and in aspirations. The model has only three stages, the first being the most diverse; the problem or situation is viewed from as many different perspectives as possible, in order to extract a highly comprehensive understanding. The best approach is to generate lots of questions in order to interrogate the situation without closing down the enquiry too soon or to be restricted by polar-views. Stage two requires further exploration of questions deemed of particular value or simply requiring more in-depth enquiry. The third stage requires the identification of relationships and patterns. The depth of enquiry is time consuming and therefore less appropriate for forecasters working on short-range forecasts, such as those used for the fashion and textile industries.

Forecasting colour, fabric, and silhouette directions is both a fun activity and a serious undertaking because these forecasts affect a fashion business's long- and short-term decisions. The long-term direction of a company and marketing strategies are the business of managers. Short-term decisions are undertaken by designers, merchandisers and buyers who utilise the forecasts of trends in colour, fabric and silhouette to develop or buy merchandise for upcoming seasons. Short-term trends can be used effectively by fast fashion brands, whereas for colour forecasters longer term colour trend strategies are more important [127].

\subsection{Direction and speed of change}

As previously stated colour and style changes are not radical, they evolve from previous seasons. The direction of colour change, therefore, is part of the colour forecasting process and requires careful consideration. The change may be of one or more of the following: colour temperature (or colour bias), value, intensity, or hue. Colour forecasters may also determine colour change through fashion in the same way as fashion forecasters operate. The fashion forecaster, as part of their forecasting process, track fashion trends through societies in a critical manner in order to anticipate where the trend is moving to and how it is likely to change. The pace of adoption by different target markets is also a critical consideration of this process. The process itself is largely analytical and therefore uses the more objective methods of forecasting but also the forecaster uses their instinct, knowledge and experience. The tools used to evaluate the evolution of colour change are given in Section 7.1. While the fashion 
industry can influence the speed in which they decide to change their styles, colours, etc., consumers regulate the speed they are willing to adopt new trends. However, technology has influenced how consumers are now reacting and responding to a quickened pace of change through the internet. The speed of which new trends can be communicated to consumers via the internet has complemented the speed of change to fashion that is imposed upon consumers through the fast fashion business model. According to Keiser and Garner [128], fashion trends in 1998 would last approximately one year, whereas today, the lifespan of a trend may be one or two weeks in duration. Of course, this timespan relates to the influx of new styles into the retail environment where the fast fashion model is adopted; this is not the same as with large majorities of consumers adopting new trends as previous trends are discarded. However, the speed of product change and the speed of fashion communications via the internet have made it more difficult for forecasters to monitor the movement of trends and to anticipate their trajectories.

\subsection{The zeitgeist}

When looking back, past styles and colour patterns can be identified in products and built environments that reflect the 'spirit of the times'. For some eras or decades such as the art deco period, for example, there is quite a distinct style of furniture and interior design that is synonymous with that time. This is known as the spirit of the times. Often the style is copied at a later date but in a more contemporary way that may be suggestive of the past era but has a distinct flavour of its own. Historical studies of design enable forecasters to more accurately understand trend patterns, their time, and how they evolved. Putting this method into practice for the consideration of future trends is extremely important and referred to as tapping into the zeitgeist. For Brannon and Divita [129] 'the power of the zeitgeist is its ability to coordinate across product categories'. According to Commandeur, the zeitgeist is merely a concept, but one that is used by forecasters in order to 'translate the needs and emotions of society at a particular time into stories and then into colours'. In addition, it is the zeitgeist that binds together the key players of the forecasting process, that is the 'designers, manufacturers, buyers, brands, retailers and' where her thoughts differ somewhat from those who write about forecasting, 'most importantly, consumers' [130] really only concurring with the critical thinking of Diane and Cassidy [111].

In any case, forecasters need to understand how the past informs the present and the future for successful forecasting. Scanning the environment for sociocultural trends, researching larger trends, or megatrends (see Section 8), and working with the concept of the zeitgeist are early stages of the forecasting process. For some, forecasting is a self-fulfilling objective and may also be referred to as a conspiracy as the forecasts when promoted move consumers into the trend cycle. Scully and Cobb [131] state that forecasting should drive sales through the timely identification and promotion of the correct colours for a product and market. Those correct colours should 'resonate with the spirit of the times'. Furthermore, the forecasting process, through the identification of how colours will evolve season-byseason in accordance with the 'cultural zeitgeist of current and upcoming times', is essential for good forecasting. Colour moods can be identified through the zeitgeist. It may be fair to say that this is more evident when looking at historical style and trends, as Tan [132] reports that such colour moods in the present context may not necessarily translate into colour choices available on the high street. Rather than singular colours being identified colour moods appear to relate more to colour characteristics, such 
as neutrals, or vibrant colours. Interesting though, textile designer and former chair of CAUS, Jack Lenor Larsen, was reported to have stated that 'colour forecasting works (because) it is after all self-fulfilling', and because it works, it will continue to become more focused. However, the system is not without fault, as Larsen continued to say that in his 'field of interiors, CAUS came up this year with the best card ever. Still, the fault lies with the breadth of the market covered, catering both 'to the trade only' and to various important middle markets' [133]. Linton adds, 'an aesthetically pleasing forecast tends to look reliable [because] of its appeal [therefore becoming] a self-fulfilling prophecy.... in other words, what is believed tends to become true in colour forecasts just because it does in economic ones'.

Brannon \& Divita [134] believe that the zeitgeist can influence cultural preferences. For Scully and Cobb [135] cultural, social, economic and political trends influence consumer preference intrinsically through the zeitgeist. Whitfield and Whelton [136] believe that 'colour forecasting agencies work on the assumption that there is a natural order to consumer colour preferences that may reflect the zeitgeist'. While individuals and societies may have common beliefs and attitudes that are similar at any one time because products are manufactured, each will have their own thoughts on whether or not the zeitgeist is also a figment of forecasting, or even of manufacturing, as for some, trends are also manufactured where the zeitgeist may be described as being the system through which trends move. As previously noted, it is relatively easy to establish the spirit of the times in a historical context, for both style and colour. Yet establishing the current zeitgeist is more difficult, as style and colour are changed much more frequently for mass marketing purposes, and therefore there is often very little or even no evidence of any one particularly dominate colour, or colour trend to be identified among consumers at any one time, perhaps with the exception of staple colours (black, white, beige, navy, brown). In addition, perhaps because forecasters are forward-thinking and focused on providing forecast information, a body of work does not currently exist that clearly defines the colour zeitgeist, neither in historical or contemporary contexts. Such a body of work would be highly valuable to detect patterns in colour acceptance throughout time to inform future colour directions on the basis of colour evolution.

\section{Colour forecasting in practice}

For the industry, the accurate communication of trends is considered to be paramount to their business success [137] when operating within the traditional supply chain with relatively longer lead times than for the younger fast fashion model. For the forecasters, the trend package they produce, in whatever format, is essentially the product they sell or otherwise make available to their target market. While their clients are using the information as a part of their own forecasting process and for their design purposes, the forecasters are working on their next trend package.

\subsection{Colour forecasters meetings}

Consultant to ICl prior to its being acquired by AKZO in 2008, and a long-standing member of ICl's Colour Trends panel, Tom Porter [138], stated that many businesses rely very heavily on consumer change in colour tastes in order to remain in business, thus putting the onus of colour change on the general public as opposed to it being a strategic approach to doing business. For this, Porter declared that it all 
'hinges on accurate forecasting of tomorrow's trend setting colours'. According to Holland and Jones [139], the purpose of a trend meeting is to bring together a set of individuals who have differing viewpoints in order to develop a new trend. They briefly describe the process as one that is initiated and informed by the evidence of pooled research that is broken down in order to extrapolate a number of trend concepts. Patricia Verlodt [140] president of CMG from 1991 to 1992, gave the following account of CMG's colour meetings. The group had formed in 1963 from the Inter-Society Color Council in order to address issues related to the marketing of colour. At this time the members were predominantly professionals from the textile and paint industries. They worked as a network to exchange information. The group numbers increased as the objective of the group changed to exchange trend and forecast information. The membership became more diverse which was considered to be of great benefit to the members. Membership by the time of Verlodt's presidency was around 1,000. Twice per year, a conference was held for the members who were designers, consultants, colourists, technicians, educators, researchers and marketers. Key speakers were invited to each colour meeting and members of colour organisations gave presentations to CMG members. Workshops were held in the conferences to forecast colours for a season two years ahead or more. These consisted of two groups Consumer Color Directions and Contract Color Directions. The resulting colour palettes were known as the Color Directions palettes which were made available to all of the 12,000 international members. Verlodt states that each member has an involvement in the decisions made for producing the Colour Directions palette, though details of how are not given. Members forecasting colours for shorter-range forecasts, i.e. those under two years, formulated the Colors Current palette. A process was used to track current colours that were presented as the forecast in the previous conference. The tracking process was designed in order to validate the forecast palette which formed the CMG's methodology for many years. The workshop, called Design Influences, observed all of the possible influences that could impact on colour. In those workshops, the members used demographic and economic data, plus the designs that the members believed had impact on design trends. The findings were disseminated among all of the members. A further group known as the Color Combinations Workshop, which included the Consumer and Contract members, would meet at different times throughout the year to explore colour combinations. Marketing workshops were also conducted to explore market research and to form presentations. The fashion members would meet during the summer and winter to develop colour stories in relation to the production lead times of the supply chain. The fashion forecast palette was presented at the next conference. International trade fairs and information were also used by the members. According to Verlodt, the Colour Directions produced by CMG do not specifically predict or forecast colours but were used as a guide for colour direction. Tim Hulse [141] reported a similar method used by the British Textile Colour Group (BTCG) through an interview with its founder member Joanne Bowering. It would appear that the process has not really changed since the early 1990s, as Tan [142] gives a similar account is given by Tan [134] regarding the Trend Table, which is part of the annual international textile trade fair Trend Forum of Heimtextil, Frankfurt, where leading designers from Italy, Germany, France, UK, the Netherlands and Japan meet to examine the season's trends. Here though, interestingly, each year one of the countries take turns in taking the lead.

Anne Marie Commandeur is a member of the Trend Table; she gives some insight into colour forecasting, what she claims to be one of the least-understood creative-industry professions, explaining 
why colour forecasting is essential for the consumer goods market. The Trend Table members also give presentations explaining their perspectives on 'colour, design and innovations' and use 'round table sessions' to examine similarities. While quite different sets of information and colours come to the table, there is said to be a substantial overlap with as around ' $60-70 \%$ of the samples' being identical, which is probably not so coincidental if they are taking inspiration and colour samples from the same, or similar sources. After all, if manufacturers and retailers have adhered to previous colour forecasts then the same colours will be more readily available, globally, to source colour from. Furthermore, all participants have similar feelings regarding the influencing social and technological 'undercurrents'. The team then develop 'a selection of Heimtextil trends' which is translated into a 'slideshow, a trend film, a trend book and the Trend Forum' working with 'real materials and not just a colour system, because colour is indissolubly linked with the gloss, structure and weight of a material'. Once the team has 'decided' on the colours to be included, they 'employ a colour system that's used to communicate and print the shades'. This system however, is not explained. It is reported that the team share 'thoughts and research on sociocultural developments and their likely influence on the market' as well as 'samples of textiles and colours to get a view of progress and opportunities related to innovative technology in fibres and finishing processes; it's also about cultural heritage and crafts. Past, present and future all come to the table'. The team develop a number of colour cards for different products and marketplaces and note that colour preferences are different between different countries. For example, pastels and black appear to be preferred in the UK, and vivid colours are preferred in the USA. While it is declared that the industry manufacturers are not influential in the process the team takes the marketplace into account, as well as the general public that the colour forecasts are intended to be for. They believe that some colour stories and colour trends are more apparent than others once the colours are applied to the commercial context. For this reason, the team use materials provided by manufacturers. At the trade fair, the colour trends are then presented through the Trend Forum using commercial fabric samples. Manufacturers then select colours to produce a substantial enough sample size for the fair and go into full production once orders have been secured [143].

Porter [144] gave a brief account of the importance of the dissemination of colour forecasts within trade fairs as draw together colour predictions from a variety of sources', presenting the example of Premiere Vision (PV) where 'future colour trends are formulated from consultations between European forecasters. Once determined, the colours are then displayed on large presentation boards in the Hall of Prediction. Similar consultations between experts in different fields occur all over the world', such as 'The Colour Group [which is] affiliated to Chartered Society of Designers, London' who compile their colour predictions 'under topical issues or themes [with] a representative palette of the following year's colours being culled from each sub-palette'. Prediction techniques are used including 'a street-wise approach' which includes 'visiting the key international trade fairs, an analysis of recurrent hues found in design magazines and colour associations with successful museum exhibitions, or with box office success in the theatre and cinema etc. The cinema in particular can exert powerful influence on colour fashion'.

Further meeting types include think tanks and seminars. For trend forecasting, think tanks are used to explore a product area or a season often two years ahead of the retail season. The process begins with deliberations of the current and projected macro trends. The participants are normally specialists in the 
area under consideration, for example, for colour forecasting only colour specialists will be in attendance which is different to the general meetings considered above. Seminars are a common format when a speaker is invited to give a perspective on the subject matter at hand. While this is generally a one-way communication method, audiences can find this particularly insightful to feed into further processes. For trend forecasting, the viewpoints of experts in relation to cultural shifts that will impact on the zeitgeist are highly valuable. In addition, the TED Talks seminars are also used for insights. Some meetings incorporate the physical and the digital worlds which may use Skype to bring members together from a range of locations or social media and video may be used as an integral part of the hybrid meeting. The PechaKucha presentation method whereby, presenters talk for 20 seconds on each of 20 slides they present has also become a popular means of sharing information and is now used extensively by the trend industry in order to swiftly put across their key concepts for the trends under consideration [145].

\subsection{The role of industry and traditional timelines}

Colour trends are used by professionals throughout the fashion and textile industry and related industries such as cosmetics, interiors and the automobile industry [146]. Due to the constant need for change to drive sales, forecasting has established importance in the fashion design process and in the fashion system. The nature of colour forecasting by agencies such as CMG that employ meetings and committees in their forecasting method, as described above, networking with participating professionals has become embedded into the creation of the initial colour cards that are disseminated to the industry. The colour cards are used by professionals in order to create their own colour forecasts for their products and their own shade cards. Traditionally the fibre and yarn producers were the first to use the trend information [147]. However, retailers now have much closer relationships with manufacturers particularly when using the less conventional methods of manufacture and retail, such as the fast fashion supply chain model. Due to this decreasing of the fashion cycle, perhaps a more condensed forecast cycle would be more appropriate.

The colour and trend-forecasting timeline is eighteen to twenty-four months ahead of the retail season. This is when forecasting companies such as Intercolor and CMG are working on their colour palettes for the forthcoming season. The colour-forecast agencies Color Box and Donegar Creative Services disseminate their forecasts around six months after the Color Marketing Group (CMG) and the Color Association of the United States (CAUS), having deliberated CMG and CAUS forecasts for their own clients' different target markets and price points [148]. Colour forecasters operating at the initial level engage with other forecasters and fashion and textile industry professionals through their meetings, committees and conferences as described earlier. The colour forecasters therefore consult with the mills, spinners and large brands and also with product developers and designers as part of the development stage for their new forecast concepts. Approximately fifteen months ahead of the retail season, the colour forecasters are often involved in the textile shows' trend committees, where they advise personnel in the mills on sample dyeing and contribute to the development of the colour cards for the show. It is essential that a strong colour consensus is presented to the show's attendees in order to promote the key colour forecasts for the season [149]. 
The fibre and yarn producers promote their products at trade fairs such as Expofil, where products from the apparel and clothing industries and textile industries including fabrics and yarns are showcased [150], along with their shade cards. The fairs may be approximately ten to fifteen months ahead of the retail season [151]. According to Kim, Fiore \& Kim [152], 'textile forecasting', of which colour forecasting is a part, begins around eighteen months ahead of the retail season. This would allow three to six months for the fibre manufacturers to dye threads and fibre to their required shades in relation to their own colour forecasts and colour story narratives.

Knitwear and fabric manufacturers also develop their own colour forecasts either as a separate task to the design process or as an integral part of this. They use the shade cards of the fibre and yarn companies in addition to the range of colour forecasts and trend forecasts from the forecasting sector. The fabric manufacturers promote their ranges at fabric shows, such as Premiere Vision (PV), approximately twelve months ahead of the retail season. Retailers and designers attend the fabric shows for inspiration and to connect with suppliers. They use information that they have gathered from the shows along with other forecasting information and further inspirational data from the array of other sources outlined in Section 3.2 to create their designs and product ranges [153].

Forecasting for fashion trends, or style forecasting, begins approximately one year ahead of the retail season [154]. Style forecasting is undertaken by garment manufacturers and knitwear designers. Again, the larger retailers are often also involved in this process as they work more closely with the manufacturers in order to obtain products that better meet their own consumer target market. According to Michaud [155], forecasting is the 'lynchpin' of the manufacturing cycle as it is a critical part of the planning and scheduling of apparel. Brannon \& Divita [156] recognise how forecasting enables manufacturers and retailers to 'execute a strategy' in order to ensure the correct timing for launching innovative products.

Large brands also begin their search for fabrics around one year ahead of the retail season. Fabric swatches are an important inclusion in their colour data collection both for inspiration and as a record of what suppliers are offering for the new season. Also at this time, retailers will be developing their new concepts with design and buying teams working together to interpret forecast information. Twelve months ahead of the season, the colour forecasters often work with the buying and merchandising teams of the larger brands and may also consult with the mills, spinners and large dye companies to further ensure that the fabrics and yarns displayed in the trade shows are of the correct colours. Brands will order fabrics for prototyping their ranges from the textile shows working closely with their manufacturers. Mills will work closely with their customers to ensure that the correct colours are colour matched for dyeing. Accordingly, the colour forecasters will continue to observe the movement of trends in order to bring any deviations from their forecasts to the attention of their clients. At approximately ten months ahead of the season the retailers will be working closely with their suppliers and manufacturers approving colour samples for their prototypes. Around two months later the prototypes will be approved and further samples will be made in the full range of seasonal colours to be offered to the consumer. Six months before the selling season colour forecasters may engage again with the larger brands confirming colours for production. At this stage the colours may be further influenced by designer collections. Companies' that source and manufacture locally, such as those using a 
vertically-integrated fast-fashion system, are able to respond quickly to change or to be directed by collections and celebrities at late notice, as they have full control over their logistics. Three months ahead of the selling season the garments are inspected, packed and distributed. At this point fastfashion brands will place their production orders with local manufacturers, if they are not using their own manufacturing plants. Buyers and merchandisers work together on colour ways while manufacturers colour match trims. The fast-fashion brands will be working with local companies, or inhouse to develop their colours, fabrics and prototypes using inspiration from designer collections. Approximately one month ahead of the beginning of the retail season the fast fashion brands will be shipping and distributing their products through their own logistics system [157].

Anniss, reporting for the New York Times [158], spoke of the value of brands owning their own manufacturing units therefore having full control over the fashion design process, the production process, the logistics and distribution. This enables the brand to operate within shorter production lead times and enables the company to respond immediately to changing tastes and customer preferences or desires. The system limits the risk that the trend forecasting system is still somewhat susceptible to. In the report the Managing Director of the Industry Forum Services, Britain, Ken Watson confirmed the difficulty of predicting fashion trends and consumer demand ahead of a retail season. Figure 4 below shows the traditional design, production and retail model and a fast-fashion vertically-integrated model with the inclusion of colour and trend forecasting.

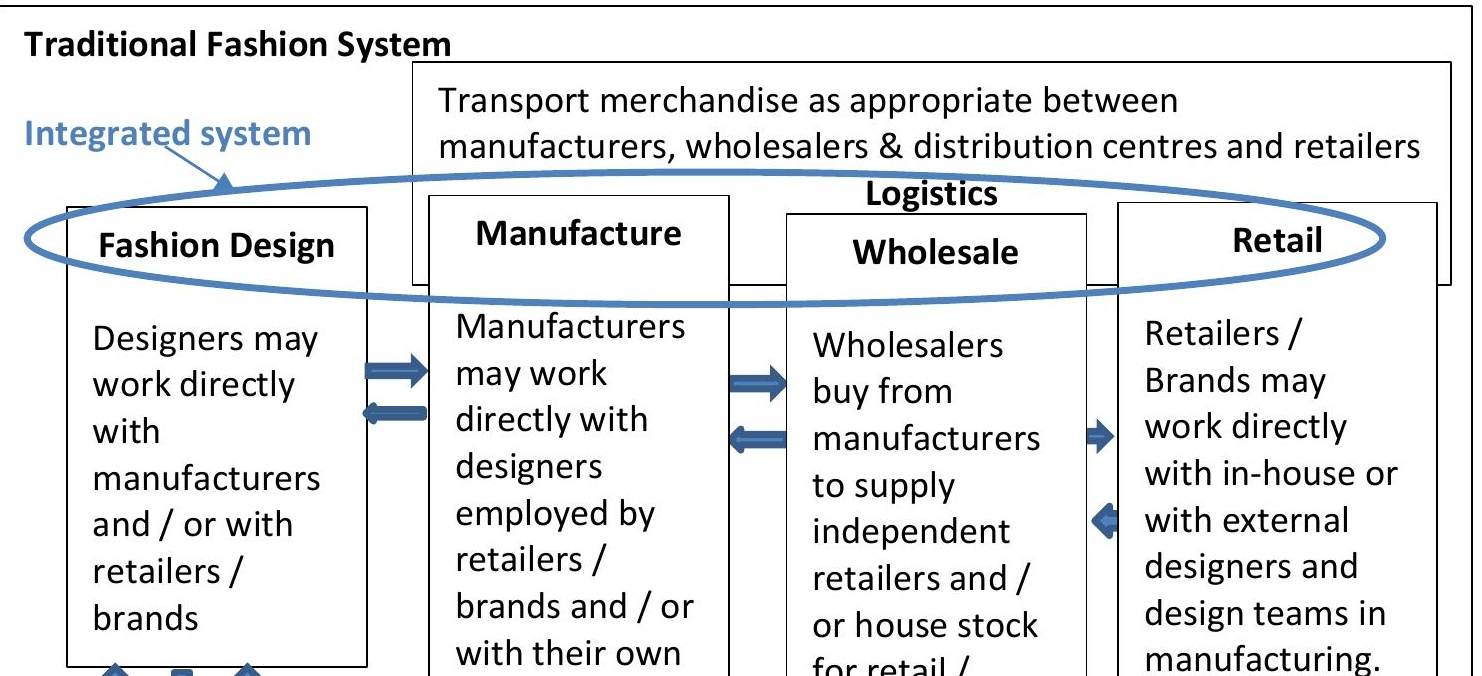


Figure 4. The fashion and forecasting system (Source: The author's own interpretation).

It can be deduced from literature that forecasters may help to increase the probability of success through the implementation of the forecasting system, by working with manufacturers and retailers on an on-going basis and by providing a consensus of colour, yet as Brannon [159] notes, 'they are not necessarily the key to success'. Other business fundamentals such as their target markets and consumers, their competitors, and the business climate all need careful attention to be paid to them. The relationship between forecasting and marketing, particularly promotion, is also very important, indeed, according to Kim, Fiore \& Kim [160] their relationship is mutually dependent which reinforces the role of forecasting in the fashion system. Edelkoort [161] believes that many consumers are happy to purchase the same style, or a similar style garment that they find comfortable or flattering, in a number of different colours. This would appear to support the notion of knowing your customers. According to McKelvey and Munslow [162] the forecasting agency Peclers, who is renowned in the industry for their colour direction also support the importance of colour in consumer products as a driver of sales. Linton [163] believes that consumers are often loyal to those brands that get the colour right for them. Consequently, it is considered that retailers who understand their customers, in terms of their desires for colour and style on a seasonal basis, may increase their success rate based on such knowledge.

\subsection{Trade fairs}

As previously stated trade fairs play an important role in the fashion system and forecasting also plays a strong role in both the fashion system and in trade fairs. The purpose of yarn and fabric trade fairs is to promote the seasonal offering and the new colour trends [164]. Interstoff is reputed to have been the first international fabric trade fair, at least in the format that we are familiar with today. It was held in Frankfurt in 1959 [165] and continues to remain one of the most important fairs of its kind. Other important fairs include Expofil, Pitti Filati, I-Textile, European Preview, Moda In, Prato Expo, Texworld \& Asia Interstoff. Premiere Vision (PV), however, has become renowned for its role and importance for 
promoting the seasonal colour forecast and key colour stories. Premiere Vision (PV) originated in Paris but is also now held in other major fashion cities around the world including New York, Tokyo, Shanghai and Moscow. Colour and fashion forecasters attend PV and other major trade fairs [166].

Premiere Vision begins with a panel member meeting at its Head Office consisting of around eighty members from all areas of the fashion industry. They work on colour palettes eighteen months ahead of the retail season grouping the colours into market sectors and further into themes. The colour palettes are developed through discussions among the panel members. Further meetings are scheduled around Europe including the UK where the agreed colour cards are presented to the fibre, yarn and fabric producers, who then send samples of their products to PV one month before the fair for approval. Each sample is colour matched against the colour card. Fabrics that do not comply with the colours on the colour card are rejected. This process ensures that a strong consensus of colour is presented at the fair. Fabrics that do comply with the colour card are displayed and used in the fair's fabric resource library. The samples are sent to Li Edelkoort who orchestrates the fair [167]. Edelkoort, is one of the most wellknown colour forecasters in the industry. She describes her trade magazines View on Colour, InView and Bloom as being some of her 'trend tools' in addition to her visual and oral presentations [168]. The magazines provide sets of seasonal themes comprising detailed stories supported by an extensive number of images; View on Colour incorporates Pantone's standard codes for precise colour reference (see Section 6.6.1). Edelkoort's team displays the samples to support the presentation. The remaining colour samples are promoted as fashion or accent colours. It is estimated that around 450 colour boards are produced for each PV fair [169]. Trade fairs therefore do not solely exist as a sales and marketing tool for fabric and yarns but are also a key tool of the wider colour forecasting process.

According to Keiser and Garner [170], many of the colour-forecasting agencies also produce fabric forecasts. Such forecasters show their forecasts at fabric trade fairs, where predictions are also delivered via seminars by expert speakers. Designers and product developers use the fabric fairs to confirm the trend information that they have obtained from other sources at the same time as selecting appropriate fabrics for their collections. For the Color Marketing Group (CMG) reports are the most used format for disseminating forecast and fashion information, as well as international trade fairs [171].

\subsection{Media}

In addition to View on Color, InView and Bloom mentioned in the previous Section, other magazines play a role in disseminating forecast information. There are a number of trade magazines and trade newspapers that further cement seasonal forecasts into the minds of the industry's professionals, such as Drapers in the UK and the US newspaper Women's Wear Daily (WWD). Industry has long-since relied on printed media, such as American Fabrics (1946-1970s, known then as the future magazine) [172], and fashion magazines, such as Vogue, have been important for disseminating trends to the general public. Trend reports are also used to disseminate information regarding the wider fashion and textile industry, such as catwalks and street fashion.

The internet has facilitated new methods of conducting business, in particular marketing and distribution, branding and advertising, and consumer engagement. Previously magazines were the 
predominant mode of information dissemination for trends to reach consumers. Today, the internet allows consumers access to current trends instantly reinforced by magazines and newspaper articles in the mass media communications network. While the use of the internet is growing amongst all age groups, generally the younger generations tend to favour the immediacy and convenience of digital media. Forecasters utilise the internet to scan fashion trends through social networking sites including Facebook, Twitter and MySpace, at speed. Through access to websites, consumer survey data and blogs, the internet has become a convenient tool for forecasters to use to develop meaningful colour stories that are unique to the retail client which serves also to strengthen the brand's identity [173].

Previously, celebrities were important endorses of fashion change, whereas today, thanks to the internet, bloggers are the new authorities on trends and products-Knight [174] describes blogging as 'a listening device, and a way to interact directly with customers one-to-one on a global scale'. Bloggers are the individuals who write and publish the blogs, the blog being the space where commentaries are written along with further factual information and product details given. According to Holland and Jones [175], bloggers have become an important part of the fashion system allowing their readers more direct access to the fashion industry. Due to this, bloggers are now viewed as powerful trend influencers. Positive reviews from bloggers on their blog sites are sought by fashion brands because of their potential to influence the popularity of a trend [176]. Many of the most-influential bloggers are sought by high-level designers and as a consequence, they are allowed to attend many of the fashion events which were once reserved for fashion professionals and fashion media personnel. Such 'A-list bloggers' are considered to be very popular amongst their followers within niche areas [177], such as fashion.

Consumers are demonstrating more trust in those personal opinions than they are of advertisements paid for by the retail brand. Images are also included in blogs, thus offering an important medium for visual stimulus for consumers. Forecasters can follow the responses to the blogs thus obtaining insights into likely trends that can then be tracked. Gatekeepers are those who 'filter the overflow of information and influence the products offered to consumers', [178]. Until more recently the 'gatekeepers' were industry professionals and the media. Today, bloggers have been heralded as the new gatekeepers. Holland and Jones [179] state that through the 'rise of the blogger', 'the flow of fashion information is evolving, given consumers and non-professionals a voice'. They have detected a particular movement in 'consumer-driven fashion influence' since bloggers appeared on the scene around 2004 and have increased their influence since their arrival. They believe that it is the bloggers' outsider views that 'resonate better with fashion consumers than the exclusive outlook of traditional fashion magazines'.

Knight [180] has identified two 'blogging personality types' that appear to resonate well with bloggers of fashion, style and trends; the signpost-blogger and the newspaper-blogger. The signpost blogger is recognised to be somewhat unusual 'in that she/he typically doesn't share her/his opinions - at least that isn't the primary reason for her/his blog's existence. A signpost blogger points out cool things of interest in her industry'. An assertion based upon signposts being used to point towards 'useful and important places' and being designed to be informative. Furthermore, Knight suggests that the signpost blogger 'informs readers about useful information and points the way to other useful information'; whereas, the newspaper blogger 'functions in many ways like a journalist - attempting to do more 
reporting than opining, she does her best to stick to the facts', as newspapers are said by that author generally to be 'impartial source[s] of information', though some may not agree.

Microblog sites such as 'Blog This' [181] and 'Tumblr' [182] are also gaining importance in the dissemination of trends. Many websites feature 'blog This' or 'Tumblr' buttons that make it easy for the viewer to save images and articles. This function allows readers and bloggers to build 'digital scrapbooks' that serve as sources of inspiration. Notes and hashtags can also be added which enables individuals to create blogs. Tumblr is a popular platform used by trend forecasters; the site is used mainly for images having very little, or no words included making it ideal for sourcing inspirations to feed into the design process. According to Holland and Jones [183] Tumblr is useful for bloggers to easily portray their own style and 'niche interests'. The image-based platform Instagram [184] is also used by individuals and by trend forecasters not least because the social media platform, like Tumblr, allows for easy sharing of ideas and inspirations visually. The networking feature enables product-consumer links to be made, giving individuals easy access to new product launches as they happen. Such 'digital sharing' has increased in popularity.

A further social media platform that has gained interest is Pinterest [185], where individuals post images on their own digital mood boards (Pinterest boards), thus helping to disseminate trends virtually. Pinterest boards are a popular medium for organising trend information and inspirational imagery. Trend services, such as Quartermaster Trends [186] (a US/ UK footwear and accessories trend agency), use Pinterest as a trend tool. In addition, although still images remain widely deployed, video clips are also used to share information digitally; such clips are known as vlogs. Vloggers can add commentaries to their videos, many of which create 'how-to' tutorials.

The internet further supports the trend forecasting sector through online commerce provision. Probably the most popular and influential online forecasting agency is Worth Global Style Network (WGSN) [187]. The service offers trend research and analysis information together with news. WGSN established in 1998, took over its rival company Stylesight in 2014 [188] thereby increasing both its number of experts and its customers. Today, WGSN reports having over 2,500 industry clients and a further 35,000 users, reaching out to the next wave of new professionals in the form of further-education and highereducation students and tutors, in 81 countries. With a team of over 200 forecasting related professionals, WGSN provide weekly and monthly reports incorporating highly detailed forecast analysis two years ahead of the retail season. In addition, they profess to track trends on a continual basis one year ahead of coming seasons. Two other influential online forecasting agencies are Trendstop UK [189] and Unique Style Platform [190]. For Trendstop the UK is growing in importance as a provider of global consensual data and as an influencer of international trends. Unique Style Platform reports on a daily basis to its fee-paying clients and provides insight reports and seasonal trend forecasts. Today, the online trend forecasting sector is further developing on account of the availability of a wealth of digital visual data and sources of inspiration from around the globe. Independent UK colour forecaster Anna Starmer, for example, provides fashion and interior furnishings trend consultancy services to clients such as Marks \& Spencer, Boden, Monsoon and John Lewis. She reports more widely through bi-annual trend books and makes images related to projects publically available through her blog [191]. Starmer is one example of many of the small independent forecasters who capitalise on the internet as a tool for 
extending their business through creating a digital presence. Currently, a part from forecasting agencies using an online business model for forecast dissemination, such as WGSN, digital media appears to be somewhat detached from the colour forecasting process itself, even though it has developed into a significant support, communication and promotion mechanism. It is logical to expect that within the very near future digital media will become more integrated into colour forecasting. There are also opportunities for research into the effectiveness and the influences of different types of digital media for improving trend forecasting methods, and also to fully realise challenges that are not necessarily as yet transparent that may be counterproductive for the system, and thus further supporting an unsustainable practice.

\subsection{Fashion forecasting organisations and their product offerings}

Many of the large colour-forecasting agencies such as CAUS and CMG are not-for-profit companies, but large colour-forecasting and marketing companies of the more-traditional type also exist, and these include Pantone Colour Institute, Colour Portfolio, Cotton Inc., International Colour Authority and Fashion Forecasting Services. Small colour-forecasting agencies and fashion-forecasting agencies are generally structured in such a way as to be profit-making companies. While the underlying philosophy of creating knowledge and colour direction for the industry should be paramount to both sets of colour forecasting bodies, for the profit-making companies there has been, and still is, growing competition amongst them for the sale of their trend forecasts. Perhaps of some comfort to these companies is the fact that most manufacturing companies and retailers will purchase a number of forecasts from different companies in order to optimise their 'chances' of success. Profit-making forecasting companies often specialise in a sector type and normally provide forecasts for style and fabrics in addition to their colour forecasts, thereby providing a more-comprehensive package. They may offer similar sets of information to all clients but packaged in different ways.

Online information provision is a growing method of both delivering the trend forecasts and as a way of doing business more generally, whilst bloggers, who are said to be 'conduits of fashion information', also provide a free additional service aimed towards the general public [192]. Generally, colour forecasters provide new colour-trend information, forecasts and direction whereas fashion- and trend-forecasters incorporate colour forecasts into their provision of more general trend information. Each provides seasonal colour palettes and colour stories, reports and narratives. Many also provide a tailored consultancy service and clients pay subscriptions to access their services; most fashion forecasting companies, for example, tailor their products to the needs of their clients, and according to Frings [193] the forecasts provided may include 'descriptions, sketches, fabric swatches, and colour samples'.

Examples of longer-serving forecasting companies include Tobe Associates, Carlin International and Donegar Group. Tobe Associates is one of the first known forecasting agencies (established in the USA in 1927), which is now part of the Donegar Group. They offer a fashion and retail consultancy service [194] and provide weekly reports aimed at the fashion retail sector [195]. By contrast, Carlin International (established in 1940), now the Carlin International Group [196] offer trend forecast information and also assist in the creation of product lines and brand strategy development for product launches. The latter activities are reported to be enabled through short-range and long-range trend analysis. Carlin predict 
emerging trends between eighteen months and two years ahead of the retail season for products and for consumer attitude trends between three to five years ahead.

Many other forecasting companies were established post-war in the twentieth century, and particularly during the 1970s. One such company is Faith Popcorn's Brain Reserve (US) which she co-founded in 1974; the core focus of the agency is on future insight and marketing [197]. According to Brannon [198] Faith Popcorn's method of forecasting included scanning periodicals, monitoring popular culture and shopping habits locally and abroad plus consumer market research. In the Popcorn Report (1991) Faith Popcorn stated that trends are predictable on the grounds of spotting a trend whilst it is starting small before gaining momentum [199]. Further companies established in the twenty-first century included Here \& There, Stylesight and Trendstop. Here \& There was soon absorbed, having merged with Donegar Creative Services in 2006 [200], prior to this, Here \& There had rapidly become a leading company in the sector for fashion forecasting also providing colour and fabric forecasts including print trends plus style forecasts. They reported online on ready-to-wear and couture collections as well as on street styles and provided images of merchandising examples across the US, Europe and Asia including in-store visual merchandising and window displays. Here \& There's tool The Color Cubicle ${ }^{\circledR}$ was used to present their seasonal colour forecasts bi-annually working on trends two years ahead of the retail season. The Color Cubicle $^{\circledR}$ gave clear and concise details of the colour stories and gave colour combinations on the lid of the box that they used to package their trend details. Yarn samples provided inside the box were intended for designers to use to inform their own colour palettes and colour ranges. Forty-five colours were presented for each season which they organised by 'family and range'. Colour cards were also inserted into the Color $\mathrm{Cubicle}^{\circledR}$, each explaining the mood and supported by imagery and details of how the colour stories could be used. Donegar Group (established in 1946 in New York) also provides trend forecasts for product and trend information to improve the wider business perspective. Their forecasting department is the Donegar Creative Services division of the business. They work on trends for colour and fabric eighteen months ahead of the retail season and, in addition, they provide current information and analysis of street trends, catwalks and retail trends. The group is renowned for their global market trend information and for providing marketing strategies to the industry. Donegar Group still use the Color Cubicle ${ }^{\circledR}$ colour tool.

Stylesight, which had established in 2003 [201], still operates in New York under its original name even though it was taken over by WGSN; they employ around 60 trend reporters who are experienced in fashion design, trend forecasting and analysis. They report on apparel manufacture and retail. The forecasting company provides colour forecasts - Colour Premiere - categorising their design inspirations into street, vintage, celebrity and denim [202]. The company Trendstop, based in London, claim that forecasting is fundamentally a form of market research as their process involves tracking early adopters of trends in order to identify a new fashion look. Through an evaluation they will determine which trends are most likely to be accepted by the majority, this they claim is based on experience. They visit clubs and 'fashion-forward stores', catwalks and young designers' fairs and observe people on the street in order to provide fashion forecasts [203].

Trend books are a common forecast package [204]. Promostyl, for example, uses trend books in order to report their colour forecasts and direction for 'textiles, prints, silhouettes and styles'. They work 
eighteen months to two years ahead of the retail season. The trend books include sketches and photography to show their design inspirations. They incorporate colour chips and fabric swatches [205]. According to Feitelberg [206], trend books are an essential element of the overall trend report. They often incorporate visual layouts and a comprehensive overview of market behaviour, trend directions including styles, colour, textiles, print, and pattern inspirations. Feitelberg considers trend boards to be even more important now than they used to be in the dissemination process.

Holland and Jones [207] provide a list of the different types of reports the trend forecasting industry makes available to fashion and textile companies. They appear to be listed in order in terms of the type of information obtained in relation to inspiration source. They state that the first report type, macro trends, reports on the 'earliest inspiration stage'. This report type is followed by catwalk reports, which offer insights and observations gleaned from whichever specific catwalk show. Colour palettes and analysis reports follow and then reports focusing on key shapes; products and design directions; trade show reports; seasonal trends; trend flash; seasonal updates; street shot photography; consumer insights; best sellers at retail; downloadable Computer Aided Design packages and clipart and finally travel guides. There is no given explanation of the content or purpose of any of these reports nor is there any indication of which forecasters produce which type of report, or their usefulness to the industry sectors. What may be inferred from the given list is that forecasters provide a number of report types possibly as a mark of differentiation from their competitors, in accordance with their target market, and possibly as add-on products for further revenue generation.

\subsubsection{Trend reporting and trend spotting}

Holland and Jones [208] discuss trend reporting and the main difference between this activity and forecasting. While trend reporting is a part of the forecasting process in terms of disseminating trend information, a trend report may not necessarily be the product of a forecaster. Trend reporters may simply report on trends in the context of what is currently available in the marketplace as opposed to having undertaken any kind of trend analysis. The report will also be characterised by the content being limited to the present rather than indicating any future direction. While trend reporting is recognised by Holland and Jones as 'an important tool' which is used by forecasting agencies and can benefit users as reports tend to track 'how retailers and consumers are responding to key trends', reporting and forecasting are not one-and-the same. Holland and Jones provide the following four reporting methods:

- 'Comp shopping - [is the method used by] retailers [to] compare how key trends are being used by their competitors - many compare how a certain colourway or take on a trend is selling or being promoted in competitors' stores'

- 'Shop windows - [is a method used when] trend services and in-house teams go to key cities / neighbourhoods to photograph which brands, colours, styles and trends are being featured in windows of influential retailers. These images help them [to] assess how well a certain trend is being received by the market, because the pieces that retailers choose to promote in their windows can have a considerable effect on consumer desire'

- 'Sales data tracking [enables retailers to] take advantage of consumer interest' 
- 'Consumer media [including] magazines, blogs [and] websites [focusing on] current available products [is a method that] can offer a useful view on which trends are likely to be important within a season. However, they are unable to predict their longevity'.

Trend spotting, on the other hand can be considered to be more akin to forecasting, though a more simplistic activity of the more complex forecasting process. Trends are reputed to be directly related to people behaviour as opposed to being the product of an industry. Therefore, Vejlgaard [209] proposes in order to recognise a trend one must 'look for signs that some aspect of human behaviour is changing'. Such signs may not necessarily be easy to identify, suggesting that the trend spotter is skilled in this respect. Vejlgaard further places the onus of trend change on consumers stating that our tastes for style change periodically, though perhaps the likelihood of such change being as rapid as seasonal as the style and colour changes that are promoted by forecasters, let alone as quickly as the fast fashion sector changes its fashion direction. The reasons for consumer taste change, according to Vejlgaard, is 'sometimes complex, and sometimes obvious, but always because of human behaviour - a part from extreme cases such as natural catastrophes - To the extent that we can understand and explain human behaviour, we can also understand and explain trends'. He continues to propose that trends are 'more predictable than most people think', owing to the fact that they are directly linked to 'sociological processes that involve human beings, and human behaviour'.

Throughout his book, Anatomy of a Trend, Vejlgaard discusses various aspects of a trend, how trends change and how to identify such change. Many of the chapters conclude with a set of 'clues' which can be used as part of the trend spotting process. However, there are some discrepancies that will be highlighted as they arise as well as points of particular interest and observations or questions that the curious-minded may also deduce. To date, Vejlgaard's proposed clues have not been contested nor otherwise critically discussed or apparently applied. Therefore, the points made in this Textile Progress that follow each of Vejlgaard's [210] clues given in quotation marks provides the first critique of his work. To begin, Vejlgaard presents the clues for spotting new and emerging trends through the characteristics of trends:

1) 'Trends are always created by people, so trend spotting is about watching people who create or are preoccupied with new and innovative styles'

This suggests that not all people are worthy of being observed, but the select few. In doing so forecasters may be missing further vital clues that may indicate the emergence of other trends or may have given earlier indications of a trend's emergence.

2) 'Trend spotting is possible because the key elements in the development of any trend is observing, either in real life or in the media. What the trend setters observe, we can also observe'

While logical, surely if observing trends in media is of relevance to forecasting what is being observed is the present, as in, it has already happened, or is happening, it is therefore the discovery of where this trend will lead to in the future that is more relevant to the forecaster.

3) 'A new trend has almost always been simmering for some time before it starts building' 
Forecasters need to identify the trend at the earliest stage possible in order to incorporate it into forecasts before it is easily observable by non-forecasters, i.e. designers, or even the general public (consumers).

4) 'If a new trend, innovative style is visible in two or more industries at the same time, it is likely to be a trend'

This would appear to contradict Holland and Jones's (2017) statement which is given later in Section 5.2.1, referring to the 'old truism' of spotting 'three examples of an idea, it is a trend', whereas two encounters they declare is merely coincidence.

5) 'A new trend is often a reaction to what has become mainstream or what has been on the market for many years'

This beckons the question, is change then more about style evolution or about the need to offer something entirely different to the current offering? Or is it more complex, where there is a need to treat each style as a separate entity, thus making a different set of changes to the different styles on offer at any one time? After all, today, many styles exist simultaneously in the marketplace and individuals often favour styles that they feel better suit them. The consequences of such implications would impact on the timescale that forecasters could operate in.

6) 'Changes in style often go from one end of the spectrum to the other' If this is case, it may suggest that forecasting could be a simple process of promoting the opposite extremity of the current style. It also discounts the previous point that would appear to relate more to slow, subtle changes through an evolutionary process. Similarly, it may be a case of treating each trend separately and therefore using multiple methods of change.

7) 'To be a successful trend spotter, you have to watch out for discrete signs of change and then put them into the analytical framework'

This sounds more complex than the six previous indicators of a trend would suggest. It also suggests that there may be forecasters who are less successful than others. The 'discrete signs of change' statement is also indicative of a consumer preference for evolutionary change. This could in effect make forecasting easier while reducing the necessary time conducting research, however follow-on trend information would be easy for clients to anticipate and would not offer fresh directions.

More detail about trend-setter characteristics are given as follows:

- 'Trend creators and trend setters are often but not exclusively found in the same visually conscious groups'

Vejlgaard does not make it clear though that trend creators may be considered as being the forecasters and / or designers, the trend setters are those being observed. It is also unclear which is the chicken, and which is the egg, and which came first!

- 'There is an over-representation of trend setters in the following groups:

- The Young, Designers, Artists, Wealthy People, Celebrities, Gay Men, Style Conscious Subcultures'

This seems to suggest that these groups are the 'select few' previously mentioned, and further suggests that ordinary people are not worthy of being watched.

- 'If one or more of these groups accept a new style, it is more likely to become a trend' This would suggest that perhaps there is some kind of homogeneity of taste among these groups of individuals. 
- 'The more trend setters in each of these groups that adopt an emerging style, the more likely it will become a trend'

Yet, because the groups represent a small segment of the population and a trend needs to be adopted by a majority to be profitable for the industry, how valuable is this method of trend spotting for the forecasters? In other words, what if the trend stays only within these groups? Forecasters must surely also evaluate the value of each trend for their clients.

- 'Trends that emerge among these trend setting groups are likely to follow the same pattern as previous trends that have emerged from the groups'

Does this suggest that trends may emerge from outside of these groups? And if so, are the 'patterns' of emergence the same?

- 'By studying those styles and taste of the trend setters, you can stay ahead of new developments in many different lifestyle areas'

However, this does not necessarily indicate that there would be any currency in the trend for the mass market that the users of forecast information would have a vested interest in.

Further clues are then given in relation to the trend process:

- 'The trend process is a social process involving six different trend groups:

- Trend setters; Trend followers; Early Mainstreamers; Mainstreamers; Late Mainstreamers; Conservatives'

For the first time Vejlgaard is recognising the importance of the general public as being a critical part of the trend process as they will appear in one of the latter five groups. He is also recognising the more generally accepted theories of adoption (see Section 7.3).

- 'The trend process starts with the trend setters and ends with the conservatives'

There is some assumption therefore that the general public will indeed adopt all trends from the trend setters.

- 'By combining insight into what is happening among the trend setters with knowledge about the trend process, it is possible to make a qualified prediction about future needs'

Surely knowledge of the Trend followers; Early Mainstreamers; Mainstreamers; Late Mainstreamers; Conservatives, is also required, particularly the three former groups as these are critical for the profitability of mass production.

The next set of clues suggest that trends only really happen in a small number of locations:

1) 'There are only a limited number of cities that have the characteristics that will make a trend spread nationally or globally'

Yet there is no evidence of any research to substantiate this claim.

2) 'The more connected a trend setting city is to the rest of the world, the greater the chance that trends from the city, the more trend setting that city will be'

This sounds like an obvious reality, and probably one that is somewhat self-fulfilling as other locations are not considered to be of equal value in the trend process.

3) 'The following cities have a history of being places where many global trends have started: Los Angeles; San Francisco; New York; London; Paris; Milan and Tokyo'

Is it coincidental that these cities are identified as trend-cities simply because they are historically the fashion capitals of the world? 
4) 'The more a type of trend emerges from a particular city, the greater the likelihood that similar trends will emerge from this city'

Similar to the previous point, is this not naturally self-fulfilling?

5) 'The trend setters cluster in certain districts within the world's big cities, often districts that have a history of being home to poly-social groups'

Again, this appears to be an assumption as there is no research evidence to verify the claim.

What is particularly interesting having discussed the role and importance of trend setters at length, Vejlgaard [211] declares that 'observing other people that they can relate to is one of the most important ways people adopt new styles' and that 'most people are more influenced by an endorsement from an ordinary person like themselves', which appears somewhat contradictory. If people in general are more influenced by other people in general, surely observing the general public is at least as much of value to the trend spotter and forecaster. Vejlgaard's next set of trend spotting clues relate back to the trend setters:

- 'A continuous product development must take place so that trend setters can continuously focus on new aspects of the style, design, or product'

Bizarrely, it would appear from this clue that trend setters take inspiration from industry, and therefore beckons the question of who is leading who. Individuals who create their own look are likely to be spotted and their style copied or interpreted by the forecasters who in turn provide the inspirations for direction change to manufacturers and retailers. The forecasters have a greater impact on the mass market than the individual trend setters can as forecasters directly influence their clients' colour choices.

- 'After some period of time, trend setters will stop being interested in the original innovative style; this typically happens when new versions of the style or product are presented to the market'

Similarly, this also suggests that the industry takes the lead in setting trends.

- 'As soon as trend setters lose interest in a trend setting product, new versions of the style or product must be available in order to regain the trend setter's interest and keep the trend process going. This keeps the trend process active and lets other trend groups become part of the trend process'

It would appear that trend setters, the same as the other groups (mainstreamers etc.) are also trend followers, in other words they do not create a style themselves to be observed for the design process.

- 'Media with trend setters' appeal must sanction the new product(s) or the new style for it to become a trend'

Again this suggests that the new product has come from the industry to be marketed to the trend setters and therefore under-values any rationale for observing trend setters as the instigators of a trend.

- 'The association of celebrities with trend setters' appeal will have a strong influence on a trend' For some, celebrities are part of the trend setting category anyway if only because they often feel that they should be copied and act as a role model for their fans.

- 'Hollywood - movies in general - will have a very strong influence on a trend'

This further substantiates why designers and forecasters use the film industry as a key source of inspiration. 
Concurring with Meyers and Grossen [212] as given later in Section 5.2, Vejlgaard [213] understands how we use our senses to engage with the world around us, relating the five senses to how trends change. For example, literature, art and design are given for our sense of sight; food and drink for our sense of taste; fragrance for our sense of smell; music and language for our senses used to hear and dance and travel for our sense of touch, or feel.

Another type of trend spotter worthy of mentioning is the cool hunter. Emerging in the 1990s cool hunters gained media attention for observing the general public on the streets and in the bars and clubs of fashionable cities. Their observations provided qualitative data for trend agencies. What was noticed in particular by the media was that often reports based on cool hunters' observations did not always translate into actual trends. According to Vejlgaard [214] this was most likely due to cool hunters perhaps not having the refined skills of the trend spotter or forecaster and having little or no knowledge of products and trends. As a consequence, cool hunters may have simply made the assumption that what they identified as being cool would naturally become a trend, simply because of its novelty.

\section{$5 \quad$ Colour forecasting skills, knowledge and their acquisition}

Having trend knowledge is a necessity for colour forecasting as is having knowledge of both colour theory and the principles of colour psychology. This Section focuses on the soft skills including intuition and awareness and on tools such as mood boards and presentation techniques.

\subsection{Colour knowledge}

The accuracy of the colours used in colour stories and colour palettes is recognised as crucial in getting colour right season-by-season for the industry, and the communication of colour must also be precise [215]. Edelkoort [216] stated that colour names had gained importance in forecasting and Brannon [217] gave reference to the use of colour names as a marketing tool, as they can be used to strengthen the mood of the forecast. Hue, intensity and value are considered to be the fundamental aspects of colour relevant to colour forecasting and colour temperature is also considered to be of great importance. Colour forecasters are expected therefore to have a sound understanding of these aspects of colour and colour theories.

\subsection{Soft techniques}

According to Perna [218] forecasters require three key skills; awareness, insight and interpretation. For Levenbach \& Cleary [219] judgement is equally important. Rousso [220] declares awareness and intuition to be the soft skills of the forecaster which are the artist's approaches as are observation and memory. Verlodt [221] recognises that while forecasters may adopt different approaches and methods to forecasting there are still specific tools that all forecasters use, these are 'the forecaster's past experience and knowledge of previous trends and intuition'. She also recognises that a design itself can often determine colour through the intended shapes, textures and size.

Sources of inspiration were described in Section 3.1 in terms of being stimuli. However, inspiration in terms of ideas, creativity and vision is an 'act of drawing in' which may be through experience and observation but is largely personal. Inspiration may come to us spontaneously or may involve a lot of 
hard work in searching for clues, or through chance, or through trial and error [222]. Inspiration, therefore, for the forecaster involves flashes of inspiration that we may call intuition, as well as ideas that are derived more through the interpretation process that may also, at times, appear to be spontaneous. Insight, on the other hand, helps the forecaster to understand where the data and the trends are leading them. Scully and Cobb [223] propose that 'knowledge is the fuel that ignites insight'. For Raymond [224], 'information and data are the stepping stones that enable you to walk towards knowledge'.

Meyers and Grossen [225] speak of the empirical world, the theoretical world and the human world. The empirical is where assumptions are made about the world being external to ourselves and that this world can be scientifically measured, observed and engaged with. The theoretical approach allows us to not only measure the world but also to understand it and to explain it, it has order, or order can be applied to it, it is the foundation of theory. The human world lies between the empirical and the theoretical, we engage with the human world through our senses, through our consciousness and through our subconscious. For the forecaster, the empirical relates to the soft tools and techniques they use which is based upon observations and experimentation, knowledge and experience. The theoretical provides tried and tested tools, techniques and methods for collecting and analysing data. In Meyer's and Grossen's approach the human world provides the forecaster with the ability to work intuitively and creatively with the data collected and engaging their senses to provide richer analysis and interpretation and to provide inspiring and innovative forecasts.

For Scully and Cobb [226], the forecasters' 'true skill(s) set lies in identifying a trend early in its development, then making the connection between an emerging trend and the wants and needs of the consumer two years on and then translating those wants and needs into colour preferences'. However, evidence of real knowledge of colour preferences is somewhat lacking, and the nuances of colour, appear to be little understood or even considered.

\subsubsection{Observation}

According to Holland and Jones [227] 'there is an old truism in the trend forecasting sector that if you spot three examples of an idea, it is a trend'. The first encounter, they declare is 'an anomaly' where one single aspect intrigues the observer's attention. The second encounter, the 'coincidence', may indicate the beginning of a trend on closer inspection. The third encounter is considered to be the signifier of the trend. It is necessary to be mindful that not all commonalities will be trends and the would-be forecaster needs to undertake a range of investigative processes. It should also be noted that there is no indication of timescale in the spotting of the three occurrences. Rather simplistically Vejlgaard [228] values the media as a good source of information from which to observe people in addition to actually observing people in situ, and as mentioned in Section 4.4, online media have facilitated this approach.

Edelkoort [229] believes that intuition can be developed or enhanced through observation skills when one continually absorbs information from the environment. The subconscious assists us in absorbing information and this happens before we become consciously aware. Forecasters train themselves to become more aware of what it is that is being observed. When making observations of colour in the 
environment, the forecaster invariably relies heavily on memory, and their memory becomes an active part of the observation process. Similarly, when colour is observed, the memory activates recognition patterns to the forecaster's consciousness and therefore makes them more aware of the repetition of the colour. However, it should be noted that not everyone has a good memory for colour and the ability to shade match by memory is even more unreliable [230]. It is therefore important for forecasters to collect hard data to support their observations as providing them with tangible evidence allows for more-accurate colour representations to be used in the development of the forecasts. As a memory aid, observations are recorded in writing using diaries and notebooks, and visual evidence is recorded using sketches, photography and collecting physical representations. Observation can therefore be viewed as being a critical 'data collection technique'; it involves 'watching, photographing, recording and reporting' not only on colour but also on consumer behaviour with the purpose of discovering something new that has the potential to develop into a trend for a large proportion of a target market. It is a process that is undertaken in many different locations over a specific period of time at the onset of the development of a new forecast, but is also an on-going activity that further validates new forecast concepts as well as previous forecasts. Forecasting companies employ a number of forecasters to work as a team or trend-spotters (see Section 4.5.1) may freelance for a range of companies and media to report on emerging trends.

The observation part of the process becomes more complex as knowledge of historical, current and previous colours in terms of availability, popularity, cycles and forecasts from memory enter the consciousness as the forecaster consciously, and perhaps also subconsciously will be processing data, analysing and evaluating within the observation process. This may also be further enhanced for those forecasters who engage at length with the more objective methods that are discussed in Sections 7 and 8. Although the different techniques, processes and skills can be discussed as if they operate individually, in practice the forecaster will be using these simultaneously at times and may therefore need to take care to engage with the observation stage in a manner that allows for data collection without judgement, preconception or premature elimination. When combining observations with other research techniques, experienced colour forecasters are expected to be able to identify both subtle and more-obvious changes in consumer preferences and lifestyle, enabling them to track trends and their movement.

The forecaster also uses observation to track the movement, direction and timing of change both in relation to colour, society and movements within cultures. Furthermore, when the forecaster becomes a participant observer, engaging with activities to better understand behaviour, beliefs and attitudes, they can explore a new trend concept in marketplace activities. The forecaster eventually shares their observations with other fashion professionals in the industry through their forecast packages informing designers, product developers, buyers, merchandisers and the media that is said 'set(s) off a chain reaction that is fashion' [231].

\subsubsection{Awareness}

The Oxford English Dictionary defines awareness as: 'Knowledge or perception of a situation or fact'. 
Edelkoort emphasised the importance of awareness in the forecasting process [232]. Forecasters are made aware of any factors that may impact on their forecasts through a range of resources such as the news and the press and through more diverse sources referred to in Section 3.1. Fiore and Kimle [233] liken the forecaster to a 'sponge' - 'soaking up every aspect of the social context, including current issues or events in the government and economy, demographics, world and national news, sports and entertainment, the arts, science and technology, health, religion and lifestyle'. Forecasters must engage with a social lifestyle in order to take advantage of all of the resources available to them to build and maintain awareness of the trends themselves and of their influences. For obvious reasons more ground can be covered when forecasters work as part of a team or within a network where information can be shared. The process of developing awareness appears to be somewhat simplistic but, in reality, it is extremely time-consuming. Where observations can be undertaken relatively quickly, awareness requires a lot of concentrated effort and engagement with many resources to see or read and hear hard and anecdotal evidence that may impact on trends emerging, growing, plateauing and declining. The process relies heavily on recorded data yet the valuable information may not be entirely explicit or easily identified, hence insight and intuition are fundamental to the process of being aware of the most meaningful information.

\subsubsection{Intuition}

The Oxford English Dictionary defines intuition as: 'The ability to understand something instinctively, without the need for conscious reasoning'.

Edelkoort [234] regards the soft skills as being the true basis of forecasting, and holds intuition in high regard believing that it is the vital key to successful colour forecasting. For Edelkoort intuition plays its part when all of the information is brought together into 'presentable and convincing colour stories', declaring that users of the the forecast such as designers and buyers should also employ their own intuition when using forecasts for their own product ranges in order to make the correct colour selection choices for their target markets. Perhaps if the colour forecasting process was better understood by the industry they would not feel the need to invest money in other people's intuition.

Vaughan [235] wrote specifically on the subject of intuition giving it a place within both the theoretical and empirical worlds. He states knowledge as having three 'degrees' - opinion, science and illumination, where the instrument of opinion is sense, the instrument of science is dialectic, and the instrument of illumination is intuition. Furthermore, intuition being the 'absolute knowledge' derived from the mind identifying a form of inner knowing with the object in question. Vaughan asserts that intuition is the 'psychological function operative in scientific inventions and discoveries'; in that intuition plays a critical role in the formulation of a hypothesis whereby providing the proof or verification lies in the realms of science and is the secondary process. He reminds us that throughout history the breakthroughs in science have been shown to have originated through intuitive insights that were later tested and verified. Michael Polanyi, a physical chemist believes that scientists identify problems guided by feelings that have a good chance of being proved correct which may be called dynamic intuition [236]. Physicist Fritjof also referred to intuition as being 'the insight and creativity' of scientific breakthroughs whilst Einstein is reputed to have recognised the role and importance of intuition in the pursuit of solving a 
problem [237]. Edlekoort [238] fears that if intuition is quantified it may potentially limit creativity for those who understand it.

\subsubsection{Interpretation of data}

Forecasters and trend spotters who interpret soft data, are expected to be receptive to change, and to make connections by using imaginative thinking while looking for trends by scanning media and through observation. Critics of trend spotters wonder why companies would pay for a service that it appears anyone can do. Although the techniques of media content analysis, interviewing and observation seem simple, they must nevertheless be carried out continuously and systematically to be effective and trend spotting depends on being able to filter the trends from the stream of information. The forecaster is looking for shifts in demographics that can restructure society; changes in industry and market structure; differences in consumer interests, values, and motivation; breakthroughs in technology and science; changes in the economic picture and alterations in political, cultural, and economic alliances between countries [239].

\subsubsection{Decision thinking and decision making}

Hiers [240] proposed seven 'special features' of decision making that set the underlying thinking apart from other types of thinking. These are set out below:

1. 'Decision thinking is a game of imperfect information, involving the future, change and human action and reaction'

While decision thinking is not acknowledged in colour forecasting and trend forecasting literature it is clear from Hier's definition of decision thinking that forecasters use decision thinking intuitively. The imperfection of the data relates very much to the gathering of the subjective information which may have unconsciously been selected in a predetermined or pre-judged manner. It also relates to previous trend forecasts that may not be so accurate. Change and human action and reaction relates to the movement of trends at both macro and micro levels.

2. 'Because none of us live outside the stream of time, we are obliged to try to predict the future - short-, medium-, and long-term - consequences of every decision before we make it, even though those consequences cannot be predicted with certainty'

For the forecaster, this prediction of consequences is part of the anticipation of consumer acceptance process and a part of the evaluation of how trends are moving and where they are moving to. The accuracy of such stages may be viewed as being the difference between successful and not so successful forecasts.

3. 'Since we cannot 'know the future', we have to hedge against our decision going wrong by trying, in advance, to create contingency plans'

As we have seen previously in Section 4, for many forecasters the end of their forecasting process does not end when the forecast has been delivered to clients. An on-going process ensues where the 
forecaster continues to monitor the movement of trends and the emergence of new trends with the intention of updating their clients of any changes along the way.

4. 'Most of the important decisions we make are not dealing with problems that will have right or wrong answers, but rather only better or worse solutions (this may aptly sum up the philosophy of trend forecasting) - hence our need for debate, devil's advocates and rigorous, dispassionate and intuitive thinking'

5. 'The results of most of our decisions will depend upon how other people react to them, and those reactions have to be predicted before we make our decisions'

This relates again to the anticipation of consumer acceptance or their anticipated reaction to the new forecasts in the form of new product offering.

6. 'Probability estimates always need to be made, again in advance of the chances of our decision succeeding'

The probability of a trend being accepted in the short-term can be more accurately anticipated and therefore colour forecasting may be more accurate when the forecasts are produced within the two years ahead of a season phase. This also substantiates why many forecasters are attempting to work even closer to the season in order to better ensure that their forecasts will be more successful.

7. 'Lastly, risk / reward calculations also have to be predicted beforehand to find out if it will be worthwhile trying to achieve our objectives - even if it is highly probable that we will successfully carry out our decision. We always need to remember before we make any decision that something not worth doing, is not worth doing well'.

Points two and three above emphasise the fact that predictions are fallible. For retailers, their contingency plans may be to include more staple colours than new fashion colours into their new product ranges or to carry successful fashion colours over a longer period of time if they are proving to be successful for their target markets. Points four and six have perhaps being addressed over more recent years as the forecasting process has developed in complexity to further include cultural, societal, political and environmental trend data. It has to be remembered that it is not only the forecasters that employ decision making and decision thinking, but also the forecast users who must interpret the data and make their own colour selection decisions. It would be of great interest to know if the same thought-, reasoning- and decision-making processes apply to both the forecaster and the information users; though it may be fair to assume that the information users are probably confined to the ideals of the forecasters if only for the sake of time-saving.

Hiers [241] recognises that we do indeed take a sense of reality from the perceptions of reality of others anyway - 'Since the decision thinking is concerned with finding practicable solutions to real problems in the real world, our perception of reality must be as accurate as possible. But we do not perceive most reality directly, rather what we perceive are our own models of reality, which we have created or borrowed from other people'. Is forecasting then simply a process that enables or ensures that everyone involved in the sectors that the forecasts relate to all perceived reality through the one lens? If so, 
whether purposeful or by default, forecasting creates homogeneity in thinking, in product, and in trends. External influencers would therefore be those who exist outside of the system but have the ability to affect it, i.e. consumers, which would justify the inclusion of street style in the forecasting process to attempt to include potential consumers who prefer to express their own individuality, either individually or as a part of a group. It also provide a reason for including demographic data, and megatrend data that is essentially based on the social, economic and cultural influences.

\subsubsection{Visual analysis}

Visual analysis requires the enquirer to establish commonalities and patterns of common factors within the items under analysis. The process relates back to theme development (Section 3.2) where the identification of a concept is of particular importance. This is achieved through the use of connection mapping, seeing and working with similarities and contradictions in the visual materials. Forecasters generally use their own method of working with this stage but will involve selecting and eliminating data. The process requires the individual to understand hidden meanings and messages from the data. This is what Gaimster [242] refers to as visual literacy - having the ability to 'read and understand imagery'. She declares that it is necessary to understand the context in which the imagery is being analysed. While this is purported to be an intellectual process it should also be viewed as an internal process, i.e. one that involves the use of intuition and insight. Images are critical ingredients for the presentation of trend information, because the summary of such material is an integral part of the forecast.

\subsection{The forecaster's tools}

The subjective tools, methods or skills used by the forecaster include observation, awareness, intuition and thought, reasoning and decision making as discussed earlier as were knowledge and visual analysis which complement those skills. The main tool used by forecasters to develop and communicate their concepts and colour stories are mood boards, a general term for mounting board usually A3 or larger, on which visual data is arranged. Mood boards used for specific purposes have names that reflect the intended purpose, such as concept or ideas boards, inspiration boards and colour story boards. The data used to create the boards draws on a large number of resources in the form of images and colour representations.

\subsubsection{Mood boards and colour boards}

Mood boards are used in order to better communicate data in a visual manner [243, 244]. They are considered to be very 'powerful tools' [245] that play a major role in the creative process [246]. Mood boards are used principally to develop concepts and ideas [247]. The contents of the boards should be meaningful and carefully selected and arranged in order to promote feelings in the onlooker by stimulating the human senses [248, 249].

For Foire and Kimle [251] the success or effectiveness of a board is due to its aesthetic appeal. Davis [252] ascribes psychological effects that have the ability to stimulate our senses and emotions as critical for mood board development. Better Product Design [253] view the 'subtleties of aesthetic experience' 
as being a strong contributing factor to the meaning of the messages that may be communicated via mood boards.

Garner and McDonagh-Philp [254] believe that mood boards, while being useful tools for idea creation, are also very useful tools for identifying and exploring a problem space and for finding appropriate solutions for the problem state. For them, the board is where the problem and its solution merge. The mood board is intended to be metaphorical rather than literal [255].

The mood board development and creation process has three stages each offering opportunities for deep levels of engagement to take place:

i) the research or data collection;

ii) selection and elimination, where ideas develop and take shape. At this point further visual data may be purposefully collected in order to develop the concept further;

iii) presentation of the board to an intended audience [250].

The first stage involves the initial inspiration and research where the data collection needs to be wide and diverse and individuals need to suppress judgement and preconceptions for creativity to flow. This stage should be stimulating, motivational and unrestricting in order to begin to explore the problem space creatively, it may or may not involve a problem finding enquiry. The second stage of the process, the development and creation of the mood board, should ideally include on-going data collection which usually becomes more refined and focused as initial ideas are further explored and developed. At this stage it is desirable to include an exploration of different media to further develop ideas and curios. The creator works with selection and elimination processes, which include analytical, interpretation and evaluation skills, to explore the solution space in relation to the problem space. This part of the process requires a deep level of engagement in order to develop creative and innovative solutions. Finally, the mood board or boards are presented to a captive audience and are usually used as a springboard to further develop design ideas into workable design solutions, which may be through the use of sketchbooks or further boards [256]. Holland and Jones [257] propose that mood boards can also be used, and are used, by forecasters to track a trend, however, the method is not elaborated on. They further propose that the images enable the viewer to understand the messages and references in relation to the trend and can associate with the visual data in order to feel the intended mood. However, some may feel that this understanding and emotive connection may not be realised by all general onlookers, but privy to those who have an understanding of more artistic ways of working, such as forecasters and designers. The mood board process is shown in Figure 5.

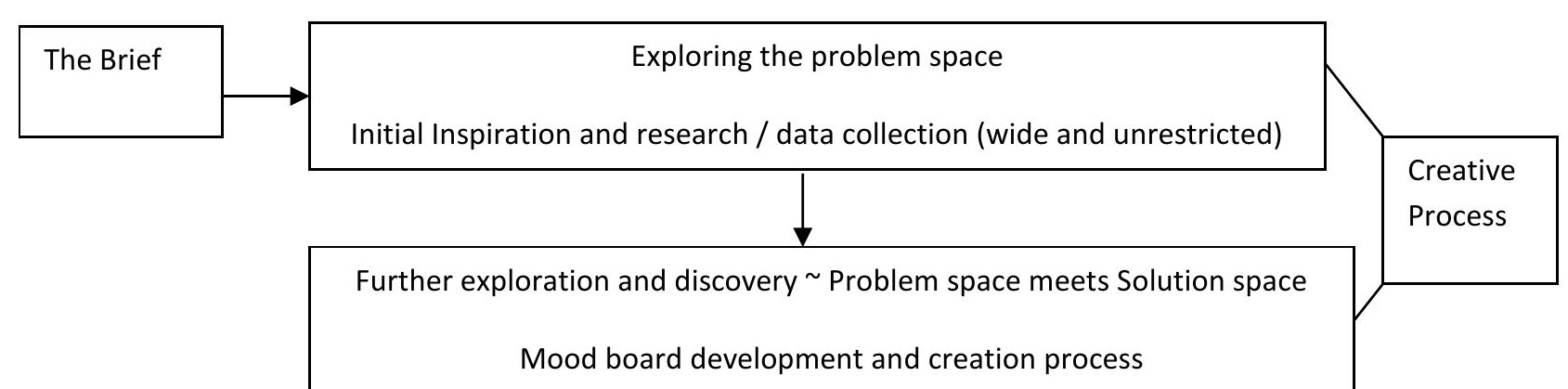


Figure 5. The mood-board process (Source: Author's own interpretation).

Colour story boards are specific to the task of colour forecast creation. Again, the boards are used to present the inspiration sources as well as indications of the colours for the forecast. An example of a colour story board is shown in Figure 6.

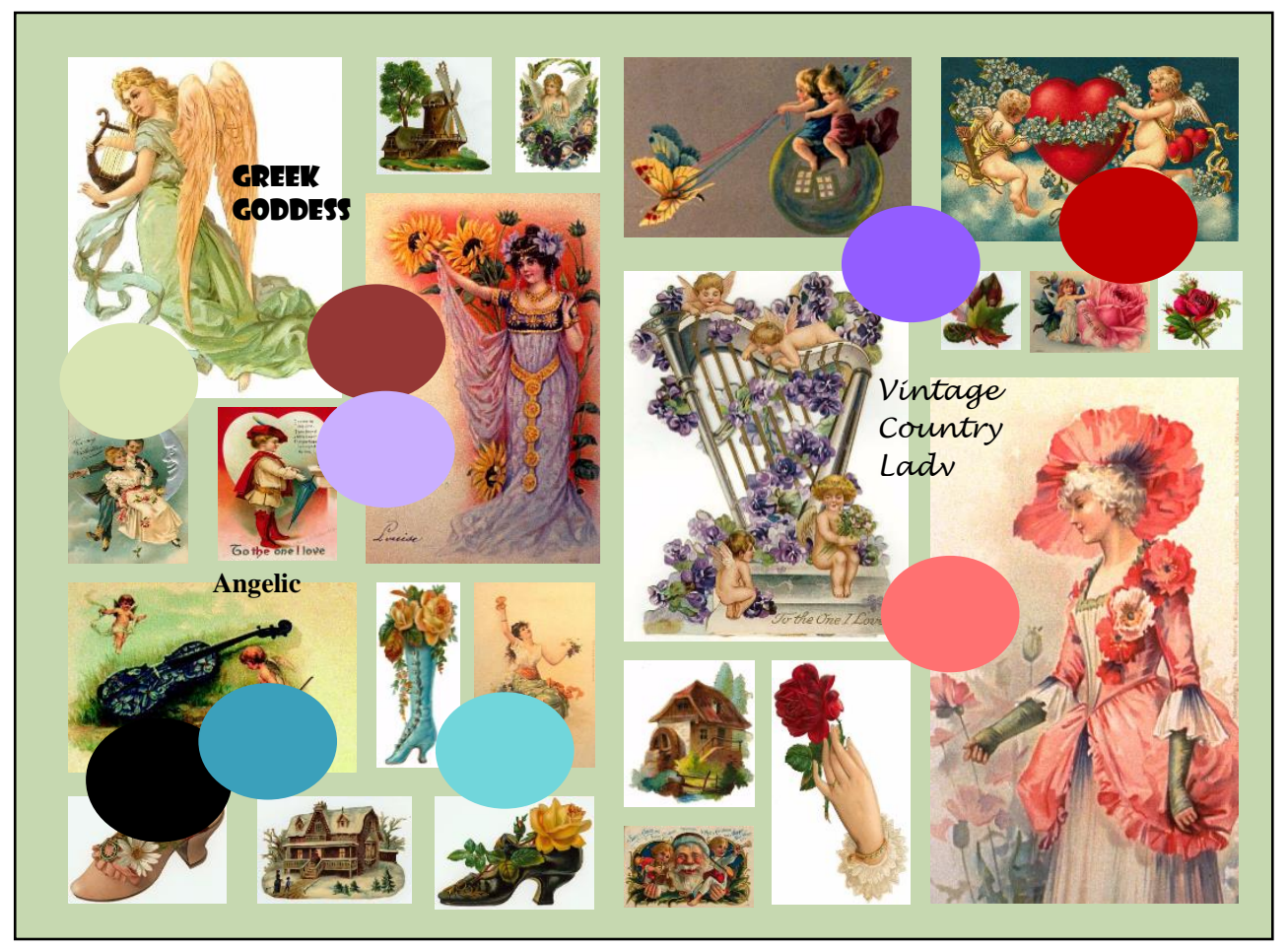

Figure 6. A colour story board (Source: Author's own work)

\subsubsection{Presentation skills}


The visual data used to develop mood boards, colour boards, and trend forecasting packages should be of sufficient quality and quantity to inform the trend forecast in order for the correct messages of the trend to be easily identified and understood. Invariably the forecaster is not physically available to discuss the visual content of the forecast and therefore written contexts and reports often accompany the forecast to ensure clarity. According to Holland and Jones [258], the forecast should inform the onlooker of the original inspiration, or inspirations, and should provide a clear rationale of how the forecast works with the season that it is intended for. On occasion a verbal presentation may also be given. This is typical of forecast presentations at international trade fairs. The oral presentation has equal importance as the visual presentation, and often works as a sales pitch to the audience, bringing them into the forecast, to see it, hear of it, feel connected with it and to envisage it in related future product. Edelkoort is particularly good at selling the trend forecast story, the rationale and the colour concepts to her audience.; such entrepreneurial skills appear to be an essential quality, as Gale \& Kaur [259] state, 'trends present real business opportunities'.

\subsection{Skills and knowledge acquisition}

Colour and trend forecasters generally have fashion industry experience, either in fashion design or fashion marketing [260] and not surprisingly therefore, trend forecasting is often incorporated into the fashion design modules of University degree courses and some may include trend forecasting in moregeneral fashion marketing modules. The skills of the forecaster are largely embedded into the activities of fashion design and fashion marketing courses but the would-be forecaster must further develop and enhance their skills on the job. During 2001 and 2002, Cassidy (nee McLuckie) [261] conducted a study observing the behaviour of two sets of students in order to develop an in-depth understanding of how colour forecasting methods and skills are taught in the UK, and how design students implement such skills and knowledge to produce colour stories for their forecasts. The observation exercise was developed comparing two modules of the same title with similar aims directed at undergraduate students taught simultaneously in two different faculties of a UK university. One module was delivered in an art and design school to fashion design students, the other to textile students in a science and engineering school. The modules were taught for the duration of one semester by different tutors. A freelance colour forecaster acted as a guest lecturer on the module for the textile students. Some interesting observations were made, firstly in the delivery of the modules and secondly, and more importantly, in the approach to the assignments by the students, as explained below. This exercise prompted questioning of the decision-making processes that influence the development of a colour story, or stories, for the purpose of colour forecasting. A more in-depth understanding of the forecasting process was established through the two case studies based on the activities of the students on each of the two modules. The important factor where colour forecasting is concerned is how convincing the colour story is as a predictive tool for the industry in terms of accurately assessing consumer demand to promote high sales volumes of fashion products. The process of colour forecasting is essentially a seasonal problem to be solved in the form of a colour story. Van Someron et al [262] refer to problem solving as 'constructing solutions and constructing justifications for these solutions', which is essentially what colour forecasters and trend forecasters do. 
In the textile module 31 students were observed. The aim of the module was to enable students to understand the role of the forecasting profession and to understand how colour prediction packages are developed. As part of their tasks they evaluated past and current trend information. The students worked in groups in the role of design teams. Mood boards were produced by each student for assessment purposes. One tutor and two guest lecturers delivered the module content and a fourth member of staff supervised the workshop sessions. Each week the three-hour allocated time period was divided into one-hour lecture time and two hours for the workshop with additional self-directed learning time. The fashion module consisted of only 13 students. The aim of the module was to enable students to understand prediction information and its role in the industry. Their task was to research and develop a prediction package for a chosen market (thereby encouraging the development of their creative presentation skills). The students were required to work independently in the role of a forecaster. Four mood boards and a professionally-designed trend-prediction package were produced by each student for assessment. One tutor delivered all of the module content. The three-hour allocated time period was used entirely for group discussions where each student presented their work so far, the rationale for the work so far and the sources of inspiration and information used. The students also had additional self-directed learning time.

The textiles students were presented with a condensed historical background of the driving forces of fashion to help them to understand the need for forecasting and its role in the industry today. They were also presented with a considerable amount of visual trend prediction information and the concept of colour evolution was discussed. The fashion students spent one session in the library engaging with actual forecast prediction packages, mostly in order to gain inspiration for the overall final presentation of their own prediction packages. Both modules appeared to work on the assumption that students had previous colour knowledge. However, through a short questionnaire survey it was found that their colour knowledge could be considered inadequate for a potential career in the colour forecasting sector.

By the second week of the observations, it became evident that the fashion students were becoming a more interesting group to study as the module was run to a tighter schedule and the assignment task was more involved and individually developed. Also due to the relatively small number of students it was easier to conduct in-depth observations of their working methods. A second case study was therefore conducted focusing on the fashion students. Their colour-forecasting methods varied as shown below:

Student 1: Thought of the overall theme and the four sub-themes before conducting the research and data collection stage from which the colour palette was developed, the themes were then renamed and key words decided.

Student 2: Began with the research and data collection stage, from which appealing images were selected and the development of the colour palette, then developed the themes and decided on colour names while conducting further research, though focusing mostly on fabrics and then refined the colour palette. 
Student 3: Thought about a company name and logo first of all for the project and then explored lifestyle as an inspiration, largely through paintings, art, clothing and interior design images, the research and data collection stage then began at the same time as developing the themes, followed by the colour palette development and finally refining the themes.

Student 4: Thought about the intended target market first of all, then thought about the themes while conducting the research and data collection stage, this led to further research and the development of the colour palette, a logo for the company and keywords for the themes were then finalised.

Student 5: Began with the research and data collection stage and selected images felt to be appealing, then thought through the themes based on inspirational sources before developing the themes and then the colour palette.

Student 6: Thought about the overall theme first and decided on what type of images to search for conducting a very focused search but encountered difficulty in sourcing the images that it was hoped could be found; also experienced colour matching issues as the student had clear ideas of the quality of the colours to use and then felt the need to rethink the themes.

Student 7: Began with the research and data collection stage before thinking about the themes and developed the colour palette, associated lifestyles were then thought through and key words decided on before refining the themes.

Student 8: Thought about the overall themes and the four sub-themes, refined the titles and decided to take inspiration from interior design before conducting the research and data collection stage from which the colour palettes were developed.

Student 9: Thought about the overall theme and four sub-themes first and while conducting the research and data collection stage changed the themes twice before refining the thinking and conducting further research and then the colour palette.

Student 10: Thought about the themes and what kind of fabrics would be needed then started by developing the colour palette before conducting the research and data collection stage and then refining the themes.

Student 11: Thought about a niche market first and the overall theme and the four sub-themes, experiencing problems finding suitable images therefore refining the themes while conducting further research and data collection; the colour palette was then developed and theme titles decided on. 
Student 12: Collected fabrics first without any firm ideas about the themes, then thought about what the colour palette should look like before developing it from a limited research and data collection stage.

Student 13: Thought a lot about the overall theme and the four sub-themes, changed the themes and colour ideas before conducting a limited research and data collection stage while thinking about the overall trend prediction package.

While the approaches and their success varied, all students developed their mood boards in a similar manner by juxtaposing images to develop some kind of meaning. The more successful students were those who conducted a wide research and data collection stage and reserved judgement of their themes and colour palettes taking inspiration from their data collection. The success of the messages and forecast directions from the mood boards were relative to having a large number of images to select from. This also enabled students to produce more meaningful colour palettes. The process was not repeated at any other time in any other module and therefore students were not given opportunities to refine their skills or to learn from their previous mistakes, after all, the general focus of the programme was on fashion design not on trend forecasting. Furthermore, as there is relatively little time within a three-year programme to fully develop the skills required for colour and trend forecasting due to other skills and knowledge needing to be gained for fashion design and textile design, there is little wonder that forecasters must develop their skills quickly and effectively following entry into this particular industry sector. In order to rectify this situation, specific taught programmes focussed on trend forecasting are desirable. Scully and Cobb [263] aptly provide a truism 'colour forecasting is a craft that takes time, patience and practice'.

\section{Colour theories and colour knowledge}

This section explores the relevance of colour theories and colour knowledge as an extended part of the colour forecaster's tool kit.

\subsection{The relevance of colour theories and colour knowledge}

There are a number of colour characteristic terms that are central to the colour forecaster's knowledge and tool kit which relate directly to colour theory. According to Boeri [264] colour theory basically covers 'harmonies, chords, interactions and contrasts'. Colour is used as a general term, for the colour scientist it refers to the wavelengths of light that we perceive [265] or combinations of red, green and blue primary colour tristimuli that we experience as being colour. When talking specifically about particular colour families, such as red, blue and green, the term hue is more commonly used by colourists [266]. A given name to a hue does not automatically describe its colour quality, further colour terminology for the specific characteristics, such as tint or shade, and quantifiable measurements of those characteristics are required for this purpose. Hue generally refers to the colours on a colour wheel which incorporates the primary, secondary, intermediary, and sometimes tertiary hues that we generally give a name to (as discussed in the following Subsections). Colour can therefore be considered in relation to colour names, while we cannot visualise precisely what the actual quality of the hue is, 
colour names do at least offer some conceptual perception of the colour in question. Common colour names include pink and brown, though these are not considered to be hues as they are not family names; pinks belong to the red family and browns belong to the orange family, or to the family of neutrals.

Colours such as magenta and turquoise can be visualised more specifically but still do not suggest a particular colour quality, other than being a strong striking pink and a blue-green respectively. Individuals will therefore hold different viewpoints of what is magenta and what is turquoise, even when presented with examples. Cassidy (nee McLuckie) [267] conducted an experiment with 76 students (46 undergraduates and 30 postgraduates) to explore the common perception of magenta and turquoise, while investigating colour knowledge. Each participant was shown five red-violet colours and five bluegreen colours. They were asked to select the sample colours they perceived to be the closest representation of magenta and turquoise respectively. Two of the red-violet colours were from the violet spectrum, $5.3 \%$ selected one of these violets and $9.2 \%$ of the sample selected the other violet as their magenta. A further $10.5 \%$ selected the light red-violet, which could be described as being a magenta with added white; magenta tint. A further magenta pink, though not as light in appearance as the former, was selected by $53.9 \%$, and the most accurate magenta in relation to common colour literature such as Chijiiwa [268] and Whelan [269] was selected by $40.8 \%$ of the sample. Also, not all of the respondents were confident of selecting only one colour as being magenta, $15.8 \%$ selected two colours, and $4 \%$ selected three colours. Similarly, with turquoise, $18.4 \%$ selected two colours and $4 \%$ selected three of the five colours presented to them. Two of the colours presented as turquoise were in fact greens, one a warm yellow-green which was selected by $5.3 \%$ of the sample, the other a cooler yellow-green was selected by $4 \%$. The muted blue-green attracted $23.7 \%$ of the responses. The true turquoise representation in accordance with Chijiiwa [268] and Whelan [269] was selected by $46.1 \%$ and the predominately blue turquoise variation was selected by $47.4 \%$ of the sample. In addition, respondents were asked to indicate their level of colour knowledge, only half of the sample declared understanding colour theories, while $72.4 \%$ declared understanding the colour wheel, and $44.7 \%$ of the respondents claimed to understand both. This experiment goes someway to demonstrate the subjectivity of colour perception in relation to a given colour name even by individuals who profess to have some level of colour knowledge.

It is important to note that such colour names do not always exist in non-western languages. However, colour names are an important marketing tool for global sales of fashion-related products and are used extensively in colour-prediction packages [270]. An early example of a colour card from the British Colour Council entitled United Victory Colours, demonstrates that colour names were given to the different hues along with code numbers. It is interesting to note that of the 22 colours presented five were variations of blue (Iceland Blue, Gallant Blue, Salute Blue, Panama Blue and Independence Blue), four were variations of red (British Rose, Pan-American Red, Valor Red and Concord Red) and four were variations of green (Australian Green, Pacific Green, Russian Green and Patriot Green). A further three colours were neutrals (Atlantic Sand, Dutch Tan and Chinese Earth - though the latter looks more like a dark burgundy red) and two were greys (Air Grey and Gunpowder Grey). There was a yellow-green (Victory Gold), a yellow-orange (Honor Gold), one orange (Indian Copper), and one violet (Canadian 
Violet). As the palette suggests, United Victory Colours, the colour names relate to victory-related words and to countries involved in the war effort. The actual date of the palette is not known but would likely have been during World War II or soon afterwards. The palette colours appear relatively sombre and muted but may also have faded over time. The coding numbers are called cable numbers; it is not known what the coding system used was. Perna [271] recognised that the standard colour reference used by the Color Association of the United States (CAUS) was often used as a colour communicator but did not state how universal this, or any other similar system was. In 1999, Edelkoort [272] noted that colour names were at that time beginning to grow in importance for colour forecasts. Brannon [273] also remarked on the use of colour names as a marketing tool, stating they present the mood and refresh an otherwise dated colour. Kinning [274] notes how skilful the art of colour naming is, as requiring 'imagination and stacks of reference books'.

Colour forecasters should have a good understanding of colour composition - It should be noted that colour forecasters and fashion designers develop their understanding of colour knowledge through the use of art materials rather than through textile coloration - For example, when using artists' paints, a brown may comprise orange and black giving warmth to its appearance or may be a combination of all three primary colours (red, yellow and blue) which results in a cooler earthy tone, known as neutrals, or chromatic neutrals (see Section 6.4.2.2). The quality of neutrals changes again, as with all colours, with added white, grey or black, known as tints (or pastels), tones and shades (or darks) respectively. It is necessary to understand the basics of colour composition as this will greatly affect the manner in which juxtaposing colours appear when grouped together in a colour palette. As well as understanding colour in the sense of composition, it is also imperative for colour forecasters to understand the power of colour for the sale of products, which also substantiates the importance of accurate colour forecasting for consumer-related products, and for the fashion and textile industry sectors (Section 6.6). In addition, colour forecasters, as with all colourists, should have a sound knowledge and appreciation of how we perceive colour and the manner in which colours can appear to shift under certain conditions and should also appreciate that we do not all see or experience colour in the same way. In order to improve colour communications between colourists within the textile colouration sector and forecast / design sectors, it would be beneficial for the latter to be educated in textile colouration as well as having an art and design background; maybe then a more-rounded understanding of colour in textiles could impact on improving the design of colour palettes and on the colour forecasting process itself.

\subsection{Colour perception}

Colour is defined as 'a sensory or perceptual component of visual experience typically characterised by the attributes of brightness or lightness, hue and saturation; but in certain cases having zero saturation and so no hue' [275]. Amplitude refers to the height of the wave and determines the intensity, or brightness of the colour we experience. The higher the amplitude of a wave the brighter the colour will be. Colours with a low amplitude appear muted [276]. While knowledge of colour as a science would appear to be of no real consequence to forecasters, there are two factors that may benefit both forecasters and designers when considering colour palettes; colour deficiency (Section 6.3) and metamerism (Section 6.3.2). 
The way that individual humans see, or perceive, colour as explained by their trichromacy and trichromatic colour theory, can differ from person to person. Perception in general terms relates to the five senses which we use to understand our surroundings [277]. For Klarén and Anter [278] understanding our world is not a linear process but an activity in itself based on how we interact with our world. They refer to perception as being subjective, and that colour perception is critical to our survival as it helps us to differentiate one thing from another. Colour enables us to distinguish different forms or objects and creates our spatial awareness. Klarén and Anter continue to discuss categorical perception as being the phenomenon that helps us to 'structure our perceptions'. They suggest that while we are born with a particular genetic make-up that gives us the ability to see colour, we then learn to build an understanding of the relationships of colour, which presumably may be considered to be more unique to the individual. They estimate that humans have the ability, with optimum sight, to see almost ten million different nuances of colour, but not as individual colours, rather they are 'observed together'. We observe similarities and distinctions; a theory on which the German physiologist Ewald Hering constructed his opponent colour theory of colour vision (1878). Hering theorised four perceptual or psychological primaries, red, green, blue and yellow [279]. Klarén and Anter also declare that our experiences of harmony, balance and coherence are related directly to categorical perception, in that we respond to relationships observed in colour combinations that have the same degree of 'whiteness, blackness, or chromaticness, hue or lightness'. Simultaneous contrast is also recognised by Klarén and Anter as being part of categorical perception.

According to Martinson [280] trends and forecasts also play an important role in influencing our perceptions of colour, in particular through the zeitgeist (see Section 3.5), and infers that the colours of past times can be thought of in relation to the decades, at least of the twentieth century as colour changes were less frequent prior to this time. This would also suggest that a sense of colour culture can be identified through those popular colours at given periods of time (Section 9).

\subsection{Colour deficiency}

The light sensitive area of the retina in the eye is composed two types of photoreceptor cell, rods and cones. The rods respond to low levels of illumination but do not enable colour vision. It is the cones, which respond to high levels of illumination which enable us to perceive colour. Because there are three types of cone: S, M and L. The shorter colour wavelengths we see (blues / violets) are detected by the $S$ cone type, long wavelengths (reds) are detected by cone type $L$ and cone type $M$ is sensitive to the midrange wavelengths (greens). With overlap of sensations between the three cone types, humans also perceive the full range of intermediary colours with which we are familiar, and this is known as trichromacy [281]. If one of the cone types is faulty certain wavelengths cannot be detected, and this will affect the way an individual will perceive colour. For example, if cone type $S$ is deficient blue-violet colours are affected, if cone type $L$ is deficient red colours are affected, and if the $M$ cones are deficient green colours are affected. Around $8 \%$ of males (around the globe) are believed to have a deficiency of colour. Faulty or missing $\mathrm{M}$ cones are most common affecting the experience of reds and greens. Defective $L$ cones can also impact on an individuals' ability to see red and green colours. In more-rare cases the $\mathrm{S}$ cones are faulty or missing affecting the experience of blues and yellows. Colour deficiency is often incorrectly referred to as colour blindness. Colour blindness only occurs when two cone types are 
faulty or missing which is extremely rare. Colour experts, often with the exception of forecasters and fashion designers, are commonly tested for colour deficiency (as this negative impact on dyeing and colour matching) and most often the Ishihara test is used [282]. It involves a system of 38 plates wherein on each plate a number or a randomly formed shape appears as a series of coloured dots embedded within a further series of background dots [283]. An example of one of the plates shown in Figure 7 showing the number 74 .

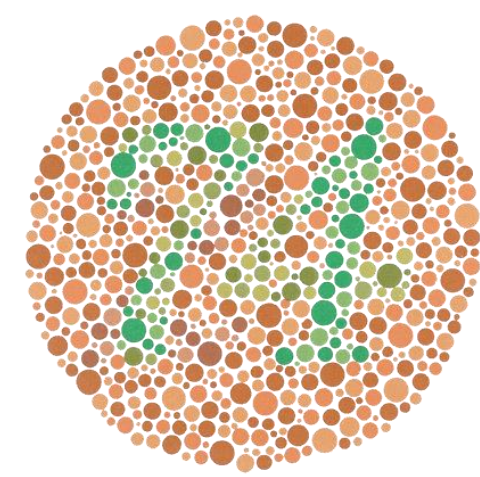

Figure 7. An example of an Ishihara test plate [284].

Further tests that measure an individual's perception of colour include the HVC Colour Aptitude Test and the Farnsworth-Munsell 100-Hue Test. The HVC test is similar to the Ishihara test [285]. The Farnsworth-Munsell 100 Hue Test is the more sophisticated; the observer must place the colour representatives in the correct order, otherwise known as an arrangement test. The results will determine whether a person's vision is classified as being superior or average, or having low colour discriminancy ability [285] but test/retest trials have shown that the tests are only able to reliably classify observers into very broad performance bands [286]. While individuals who work as colour matchers in the dyeing industry are tested for any colour deficiencies, others who work in the fashion and textile design sectors such as designers, buyers and forecasters, are not tested as a matter of course for their respective job roles (unlike textile technologists who generally are); nor are the students tested who may work in the sector in the future. Therefore, colour forecasters who are prescribing colours to industry are quite likely to be unaware of any colour inconsistencies between individuals, or themselves. Colour inconsistencies of this nature are known as observer metamerism [287]. Metamerism is 'the phenomenon wherein two coloured samples will appear to be of the same shade under one light source but will appear to be different shades under a second source' [288].

\subsubsection{Simultaneous contrast colour shifts}

Klarén and Anter [289] speak of our normal vision being that we naturally experience the wide number of colour variations at our disposal as emerging in such a way that we only discriminate between relatively few colour nuances. In other words, 'our visual system is developed for a continuous spectrum of light and gradual changes between different light situations'. Simultaneous contrast plays with our perception of colour. In the early nineteenth century Michel-Eugène Chevreul researched extensively into the manner of which colour shifts are realised when certain colours and colour qualities are juxtaposed. For example, a colour with a light value appears to be much lighter when placed next to a 
colour with a dark value. Chevreul concluded in his work that 'certain colour combinations fostered illusions of hue, value and intensity in Humans altering their colour perception'. This visual response is known to be caused by the afterimages we experience when observing colour. Chevreul [290] provides four basic rules of after-image:

1) 'High-intensity colours induce high-intensity afterimages

2) Low-intensity colours induce low-intensity afterimages

3) Dark values induce light afterimages

4) Light values induce dark afterimages'

Furthermore, in 1839 Chevreul gave fourteen 'conditions' relating to colour contrast and afterimage in his definitive work 'On the Laws of Simultaneous Contrast' as being:

1) 'Colours are modified in appearance by their proximity to other colours

2) All colours seem lighter and more dramatic against black

3) All colours seem cooler and more subdued against white (most colour forecasts present colour swatches, wrappings and chips on white pages)

4) Dark colours look darker against light colours than against dark colours

5) Light colours look lighter against dark colours than against light colours

6) Colours are influenced in hue by adjacent colours, each colouring its neighbour with its own complement

7) If two complementary colours lie side-by-side, the contrast makes each seem more intense than it looks by itself (Eiseman [291] also notes that complementary colours 'complete each other' as one is a warm hue while the other is a cool hue. In addition, when juxtaposed, each complementary colour appears to vibrate at the point where the colours meet. McLuckie [292] describes this as a 'tinge' at the edges of the hue).

8) Dark hues on a dark background that is not complementary appear weaker, or less intense, than they do on a complementary background

9) Light colours on a light background that is not complementary appear weaker, or less intense, than they do on a complementary background

10) A brighter colour set against a dull colour of the same hue further deadens the dull colour

11) When a bright colour is set against a dull colour, the contrast is strongest when the latter is complementary

12) Light colours on light backgrounds that are not complementary can be greatly strengthened if bounded by narrow bands of black or of complementary colours

13) Dark colours on dark backgrounds that are not complementary can be greatly strengthened if bounded by narrow bands of white or of light colours

14) The greatest afterimage appears when figure and background relationships have the same value, and when a large background is set behind a small foreground figure'

Generally, as noted against point 3 above, the colours in colour forecasts are presented on a white background. Colours may be isolated or juxtaposed. While colour forecasters may 'play' with the presentation of the colours for visual affect in relation to reinforcing the message of the theme (see 
Section 3.2), there is no evidence in the prediction packages of suggesting any particular colour-ways, which is left to the user of the trend information, i.e. designers, buyers, etc. Colour shifts therefore appear to be of little consideration to colour forecasters, yet the colours they are proposing to harmonise as colour-ways may be problematic. It has been identified by Diane and Cassidy [293], that although a range of colours are promoted as being the trend colours for a given season, retailers and designers tend to lean towards using a very small number of those colours and may choose to alter them accordingly for the preferences of their target market, or target consumer. In retrospect, this adjustment of colour may be due to the fact that when using the forecasters' colours together may be causing unwanted colour shifts that are unappealing; whether the designer is aware of the science behind the unpleasantness or not. Invariably, the colour forecasters leave the colour selections for products to the designers (or other users of the trend information), but the time that the designer needs to explore the effects of their colour choices may prove costly; both in terms of their time and in terms of getting it wrong for the consumer. This lack of efficiency may therefore render the colour forecasts somewhat useless, or non-cost effective. Colour forecasts could therefore be more informative and valuable if some kind of colour analysis of the trend colours accompanied the forecasts. Another point to note is that colour forecasters will spend considerable time delivering the message about the precise colours in their forecasts, only for the trend user to manipulate the colour to suit their target consumer. This raises the question as to why such colour accuracy is demanded by the colour forecasters in the first place, if not to exploit their clients for the ongoing sales of their trend packages. Also, why in this case, do the trend-forecast users not feel confident in their own colour convictions in the first place?

\subsubsection{Metamerism}

The perceived quality of colour can be affected by the quality of different light sources or lighting conditions, an effect which is referred to as metamerism [294]. Daylight will change due to the time of day, weather conditions and the geographic location. Artificial lights can also have different spectral compositions and qualities of reflectance. In other words, colours observed in natural daylight and within the home can appear different to the colours we perceived the item to be when considering a purchase in-store, an effect known as illuminant metamerism. Colour may also appear different when observed from different angles, which is known as geometric metamerism, and a further type is known as field-size metamerism, which occurs when colour changes become more evident when comparing colours seen from a distance with close up. Illuminant metamerism can be easily observed using a light box with a number of lighting options as seen in Figure 8 , which demonstrates how changing the quality of the light in the box affects the colour perceived by the onlooker. There is no evidence to demonstrate the colour forecasters' understanding and consideration of metamerism. 


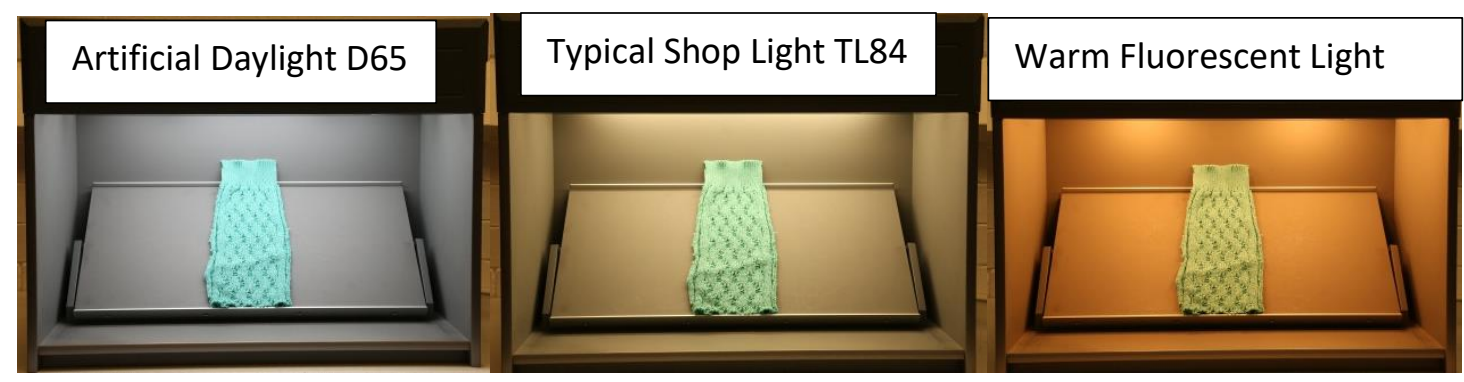

Figure 8. Light box showing the same sample of fabric under three different lighting conditions. (Source: Author's own photographs).

The observer can have an effect - two individuals may perceive colour differently due to their own colour vision or sensitivity to colour, and this is known as observer metamerism. Colour matching/mismatching is also affected by colour constancy or colour inconstancy. When a colour matches a standard regardless of the lighting conditions it is said to have colour constancy. Consequently, if the colour changes under different light sources it is said to exhibit inconstancy, which Keiser \& Garner [295] note as being due to the hue of the fabric or item. Red-orange and bright blues are known to show the highest inconstancy levels [296].

\subsection{Colour mixing}

Colour becomes more complex for the colour forecaster when dealing not only with coloured pigments, as in physical colour palettes in traditional-style colour prediction packages, but also with colour on a computer screen for the online delivery of colour prediction information. This is due to the manner in which colour mixing occurs with pigment (dyes, paints) and coloured light as used by Computer Aided Design (CAD) packages; the former is based on subtractive colour mixing which progressively reduces the amount of light reflected whereas the latter works by additive colour mixing whereby with more mixing more light is reflected producing lighter, brighter results.

\subsubsection{The colour wheel}

The colour wheel (shown in Figure 9) is a tool which enables the basic principles of colour mixing to be understood. It is also useful for identifying colour relationships, for building colour combinations and in colour stories. The twelve-hue colour wheel is the one most-commonly used as the primary colours, secondary colours, intermediary colours and tertiary colours (see Section 6.4.2) are all represented. The six-hue colour wheel comprises only the primary and secondary colours and its use is therefore considered to be very limited. Other colour wheels also exist mostly for the purpose of quantifying colour. These are discussed in Section 6.6.1.

Whelen [297] provides a useful variation of the twelve-hue colour wheel which comprises the established twelve hues: red, red-orange, orange, yellow-orange, yellow, yellow-green, green, bluegreen, blue, blue-violet, violet and red-violet at the centre of three further layers extending outwards that are varying degrees of shades (a hue with an amount of added black) and four further layers extending inwards of the circle which are varying degrees of tints (a hue with an amount of added white). The wheel therefore shows 96 colours in total. This serves as a good reminder that the wheel is 
only a visual representation of the gamut of around 7,000 hues at our disposal. Tones, however are not represented. Shades, tints and tones are the common terms used by colour forecasters, designers, artists and other colourists. They are discussed more fully in the following Subsections. Whelen also provides a second format of the colour wheel in a linear chart form as an easy-reference tool. The greyscale is also added to the bottom of the chart comprising nine variations of grey from light to dark plus black, white is omitted. Lloyd [298] prefers a variation of the six-hue colour wheel which has the graduating colours of the spectrum at the centre demonstrating the wealth of intermediary colours and six labels on the outside of this circular band of colour denoting a 'red range, orange range, yellow range, green range, blue range and violet range'. Lloyd links this to Newton's spectral hues, though omitting indigo as a hue of its own right between blue and violet; and states that we should may be 'recognise different ways of drawing hue boundaries and [that] these need not be judged [as being] correct or incorrect'. For the sake of simplicity, the colour wheel shown in Figure 9 is used throughout the remainder of this Section to demonstrate the basic theories of colour mixing, colour composition and colour relationships that, at the very least, colour forecasters should be particularly well versed in.

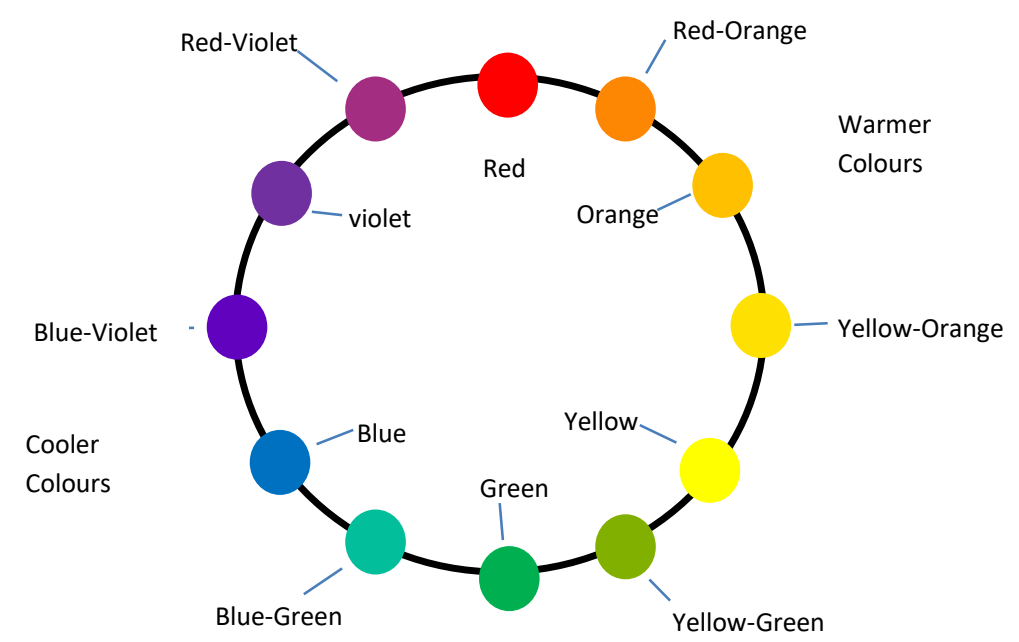

Figure 9. Colour wheel (Source: Author's own interpretation - for ease of demonstration throughout the remaining Section).

\subsubsection{Primary, secondary, intermediary and tertiary colours}

Colour is generally categorised into three basic hue types: primary, secondary and intermediary or tertiary. In principle, the additive primary colours, red, green and blue cannot be created through colour mixing; they are the natural colours found in nature, and are components of white light. In subtractive colour mixing the primary colours are cyan, magenta and yellow. Primary colours are considered to be pure [299].

Secondary colours are a mix of two subtractive primary colours. In theory, red and yellow make orange, yellow and blue make green and blue and red make purple. In reality, as each primary colour has a colour bias (see Section 6.4.2.1) the actual quality of a secondary colour is determined by impurities of other colours within that colour. The purest orange, for example, is obtained by mixing a red with a 
yellow undertone and a yellow with a red undertone. If a red with a blue undertone is mixed with a yellow which has a blue undertone a muddy orange will result. Similarly, the purest green is obtained by mixing a yellow having a blue bias and a blue with a yellow bias. The purest purple is achieved from a mix of a red with a blue bias and a blue with a red bias [300]. When mixing additive primary light colours to obtain secondary colours, red and green light mixed together make yellow, blue and green make cyan and blue and red make magenta. In theory, a secondary colour is a 50/50 mix of the two component colours, however in reality it is the visual perception of what constitutes a true secondary colour that is critical, which of course is subjective.

Tertiary colours in essence comprise all three primary colours. Technically, tertiary colours are a mix of two secondary colours and intermediary colours are a mix of one primary and one neighbouring secondary colour, the primary colour will often be the most dominant colour in the mix (refer to Figure 9). Intermediary colours are mostly referred to as being the mid-way point between the primary and secondary colour on the colour wheel as a representation of a 50/50 mix. The given names are generally red-orange, yellow-orange, yellow-green, blue-green, blue-violet and red-violet. The actual qualities of these colours are more subjective. The range of colours between the intermediaries and their neighbouring colours are also known as intermediaries. Some prefer to think of the tertiary colours as intermediary colours as the terms are often interchanged.

\subsubsection{Colour bias}

In the case of pigments, the concept of purity is idealistic as in reality all pigments will consist of at least minute traces of other colours which is known as colour bias [301]. Colour bias is more commonly known as undertone. There is some contention between designers and technologists / scientists regarding the aesthetic effect of colour bias, which is also referred to as colour temperature. Designers tend to associate more with the psychological aspects of colour and therefore describe colour appearance has having a warm or cool quality. Colour technologists and scientists tend to favour colour measurement as a basis for colour description, and refer to colour temperature with particular regard to the nature of the illuminant. However, in support of colour temperature also being an important subjective concept, Johannes Itten (1888-1967) in The Art of Colour, reports on experiments he had conducted that demonstrated that participants had physically felt cold when the room temperature was reduced to 59 degrees Fahrenheit while in a room where the walls were painted blue-green; whereas in a room where the walls were painted red-orange the participants did not report feeling cold until the room temperature was reduced to 52-54 degrees Fahrenheit. He concluded that the subjective feeling of warmth in a room painted in a warm colour compared to painted in a cool colour differed by 5 to 7 degrees [302].

Colour bias, or undertone is the subtle impurity of a subtractive primary colour [303]. When the subtleness of the undertone is weak, analysing colour composition becomes easier when comparing different colour qualities by physically putting the samples directly next to each other. In cases where one primary colour contains impurities of the other two primary colours the colour bias will move more towards the quality of a neutral hue. When the amount of impurity of a second primary colour increases the resulting hue will move towards its neighbouring intermediary colour, and becomes an intermediary colour. For this reason, for practical purposes, Lloyd's preferred six-hue colour wheel described in 
Section 6.4.1 is logical. Indeed, Cassidy [304], Wilcox [305] and Lloyd [306] very much embrace the concept of working with six subtractive primary colours: Red with a yellow bias; Red with a blue bias; Yellow with a red bias; Yellow with a blue bias; Blue with a red bias and Blue with a yellow bias. These relate directly to the following commonly used artists' paints: Cadmium Red Light, Quinacridone Violet (which is red despite its name), Cadmium Yellow Light, Hansa (Lemon) Yellow, Ultramarine Blue and Cerulean Blue respectively. These exist in order to overcome the problem of colour bias when mixing pigments to produce other colour qualities including secondary, tertiary and intermediary colours. For example, if Cadmium Red, Lemon Yellow and Cerulean Blue were selected as the only primary colour representatives, clear bright greens are possible but oranges will be rather dull and violets will be greyish-brown [307]. By first of all analysing the quality of the colours in the mix and using a sound knowledge of colour composition and colour mixing resulting colours can be better predicted and a sound understanding of colour gained. The creation of meaningful and workable colour stories and colour palettes for forecasts and for colour ways for the designer can then be fully achieved. Currently, there is no substantial, or even a partial body of research that scrutinises any colour forecaster's colour palette, or palettes to establish their validity as an effective set of colours for the design of a product.

\subsubsection{Neutrals}

When mixing artist's paints (common practice for fashion designers and colour forecasters), neutral colours, otherwise known as neutrals or earth tones, comprise all three subtractive primary colours in varying percentages. Neutrals generally have a muted opaque appearance. The actual appearance depends very much on the percentage mix of the colours and can be difficult to analyse visually because black, white or a mixture of the two may also be contained within the mix. Furthermore, when mixing artist's paints, neutrals containing large amounts of white will generally have a misty appearance. Neutrals created by mixing complementary colours are known as chromatic neutrals. In the context of fashion, Turner [308], a fashion coordinator, defines neutral colours as being 'greys, navies, black, creams and browns', and that these colours will all work well together as a tonal colour palette, whereas fashion writer Darnell [309] adds white and khaki to the list of neutrals and also proposes 'gold, silver, copper, gunmetal, bronze and pewter' as being 'metallic neutral colours'.

\subsubsection{Value and achromatics}

Value is the lightness or darkness of a colour, which according to Danger [310], is normally quoted as ranging from 0 to 10 , where black is 0 , greys range from dark to light from 1 through to 9 and white is 10. Lambert et al [311] propose that lighter greys, or values that range from $0 \%$ (white) to $25 \%$ are known as light values, or high values; and at $25 \%$ the value is known as the high middle. Greys, or values that range from $25 \%$ to $50 \%$ are known as high middle, with $50 \%$ white and $50 \%$ black being the midway point. Greys or values with added black at $50 \%$ to $75 \%$ are known as low-middle, and those with more than $75 \%$ black are known as dark values or low values. Lambert et al continue to express the importance of understanding value in design, as like hue, value creates an emotional response in the onlooker. The value of colours is often of high consideration in colour forecasting.

The given term to colours with white added is tint, with grey added they are tones, and with black added they are known as shades. Tints are also referred to as pastel colours. Tones may also be described as being muted. Tones generally have a dusty appearance whereas tints can look misty. The grey scale 
(shown in Figure 10) is a useful tool for the consideration of value. With the absence of hue, black, white and greys are known as achromatics, the opposite of chromatic [312], which is discussed in the following Section. The addition of black, white and greys can be used in any of the colour schemes given in Section 6.5. Danger [313] provides a simple tool for understanding achromatic relationships for creating tints, tones and shades of a hue. This is referred to as the colour triangle. Danger also provides a useful colour naming tool to describe the range of tints, tones and shades of any hue. Figure 10 shows both of Danger's models superimposed.

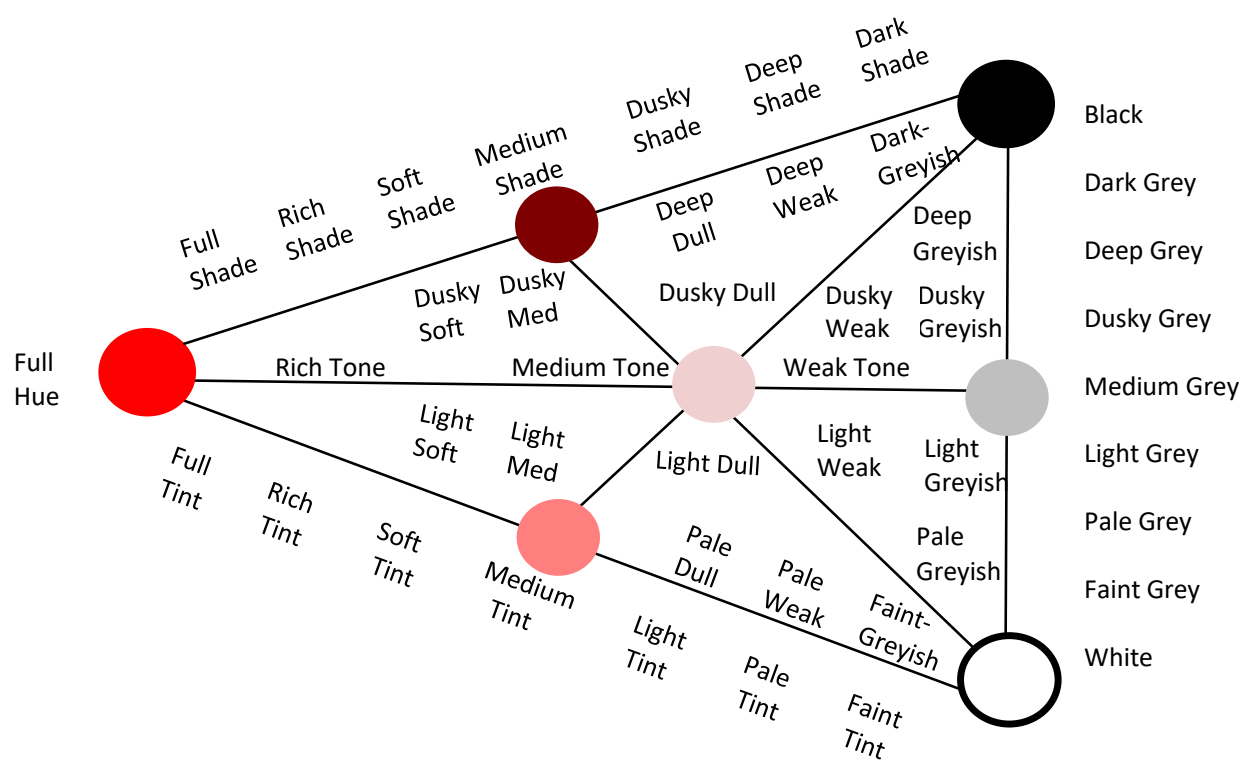

Figure 10. An adaptation of Eric Danger's colour triangle and colour naming key (Author's interpretation combining two of Danger's models to provide a more comprehensive singular tool).

Black, white and grey are used extensively in colour forecasting as staple colours. Collectively they are known as achromatics, as opposed to colours having a family name, or hue, being chromatics. Keiser and Garner [314] refer to achromatics as being neutral, some others however, consider black and white as primary colours. A wide range of greys made using varying percentages of black and white are of course possible but may incorporate a hint of colour as fashion demands..

\subsubsection{Intensity and chromatics}

Pure colours contain no traces of white, grey or black and are referred to as bright, or brights. The strength or brightness of a colour is its intensity or saturation. Pure colours (subtractive primary, secondary and intermediary colours as they appear on a colour wheel) have full strength or full saturation. Saturation is affected when the reflectance of light is reduced. Colour forecasters should be aware that the saturation of colour can change depending on the light reflectance capability of different fibres, yarns and fabric structures. High lustre flat surfaces reflect more light and will not give the perception of the high saturation of colour achievable with, for example a deeply-dyed velvet fabric. Problems still exist with some fibres in terms of how they absorb and reflect colour but restrictions on achieving certain colours on certain fibres will render those fibres unfavourable at times when the 
unobtainable colours are on-trend, or fashionable. Danger [315] describes chroma as being 'the strength of a colour, based on a scale from neutral grey to full strength' and forms the basis of the colour-naming shown in Figure 10 above. Other words commonly used for intensity, or chroma, include purity, saturation, strength and brightness.

\subsection{Colour combinations (Schemes)}

Colour combinations or colour schemes provide a theoretical method of working with colour which can be particularly useful for novice or less intuitive colour forecasters. The theories also further aid an understanding of colour and can be used as a tool for analysing colour combinations that were popular in previous decades or eras; in other words, to analyse the colour zeitgeist. Colour schemes essentially use the design principle known as harmony. In theory, harmonious colour combinations are pleasing and easy on the eye. Danger [316] provides a very basic set of principles for achieving colour harmony:

1) 'All pure colours will usually harmonise with white or black

2) Tints of all kinds will harmonise with white

3) Shades of all kinds will harmonise with black

4) Tones of all kinds will harmonise with grey'.

The colour wheel, Figure 9, is a particularly useful tool for mapping basic colour schemes and to then further develop the colour combinations as a colour story by changing the colour qualities. No evidence has been found to date to indicate that the colour palettes promoted in colour-forecast packages have been analysed in relation to colour-scheme theory; or indeed, if colour forecasters use colour scheme theories either consciously or intuitively despite which it is commonly accepted that colourists do understand colour theories and also the effects of colours used in colour combinations.

Boeri [317] and Martinson [318] recognise that colours are not seen in isolation and discuss how colours interact through changes of perception. Boeri also notes that for some theorists' colour harmony is science-based, i.e. that they work with 'principles, rules, laws, objectives and the human eye'; while for others colour is instinctive. Many colour forecasters, and other colourists, may defend the intuitive use of colour as being simply part of the creative process. Boeri makes reference to Ruskin and Albers: John Ruskin (1819-1900) was a leading English art critic, patron of art, watercolourist and writer, among other accolades [319]; Josef Albers who had trained at the Bauhaus, had devised a way to teach colour through experimentation and observation techniques including experiencing colour, i.e. simultaneous contrast (which discussed in Section 6.3.1), and is reported to have expressed the need for having aneye-for-colour rather than simply applying theories. In addition, Boeri [320] believes that 'the purpose of studying relations(hips) among colour is not to achieve strict rules within which to operate but to offer a structure of support' concurring with Itten's belief, meaning for Boeri, that it is still about the usefulness of the theories.

\subsubsection{Monochromatic colour scheme}

A monochromatic colour scheme is the simplest type of colour scheme created with only one hue though it may comprise a number of variations of the colour [321]. The colours are at least very closely 
related and may have limited consumer appeal unless a very minimalistic preference is a desirable factor within the wider trend landscape.

\subsubsection{Analogous colour scheme}

An analogous colour scheme will have a common colour as they will be neighbouring colours on the colour wheel. If using a six-hue colour wheel, each primary colour has two neighbouring secondary colours that are analogous. Similarly, each secondary colour has two primary analogous colours. The colour relationship is less subtle between neighbouring colours on six-hue colour wheel than on the twelve-hue colour wheel. In reality, so long as there is a common colour in the colour scheme the colours are considered to be analogous and should therefore work together in harmony. Analogous colour schemes are also referred to as analogous dyads, triads, tetrads, pentads, etc., depending on the number of colours in the scheme. In theory, a hexad using the six-hue colour wheel would contain all three subtractive primary colours and all three secondary colours. It is not necessary for all colours in any scheme to be of pure quality.

Below is the first comprehensive analysis of McLuckie's [322] data in relation to colour preferences and colour forecasting. An experiment was conducted to test the appeal of analogous colours in knitted samples using the same knit design. Thirty respondents rated the appeal of the knit samples using a 5point Likert scale, 1 being most unappealing, the mid-way point (3) being neither appealing or unappealing, and 5 being most appealing. Eight samples were tested. The appeal of the samples was as follows: blue and violet $73.3 \%$; blue and green $60.6 \%$; red and red-violet $57.3 \%$; yellow and green $56 \%$; red and violet $50.6 \%$; yellow-green and yellow $44 \%$; orange and red $42 \%$; orange and yellow $40 \%$. The results are shown in Table 1 with additional analysis and shown visually on a colour wheel in Figure 11. Acceptance levels have been categorised for the purpose of this Textile Progress as being high when scoring $60 \%$ plus, middle when scoring $50-59 \%$ and low when scoring $49 \%$ and below. For the purpose of colour forecasting it would be more preferable to use more colour combinations in a palette that are highly acceptable to consumers.

Table 1. McLuckie's 1998 colour preference test results plus additional analysis relating to Figure 11

\begin{tabular}{|c|c|c|c|c|}
\hline $\begin{array}{c}\text { \% of } \\
\text { appeal }\end{array}$ & $\begin{array}{l}\text { Colours in the } \\
\text { combination }\end{array}$ & & Additional analysis notes & Acceptance \\
\hline $73.3 \%$ & Blue \& Violet & No Yellow & \multirow{2}{*}{$\begin{array}{l}\text { High blue dominance, primary colour and } \\
\text { within the secondary colours }\end{array}$} & \multirow[t]{2}{*}{ High } \\
\hline $60.6 \%$ & Blue \& Green & No Red & & \\
\hline $56 \%$ & Yellow \& Green & No Red & \multirow{2}{*}{$\begin{array}{l}\text { Lower blue dominance only within the } \\
\text { secondary colours }\end{array}$} & \multirow[t]{2}{*}{ Middle } \\
\hline $50.6 \%$ & Red \& Violet & No Yellow & & \\
\hline $44 \%$ & $\begin{array}{l}\text { Yellow \& Yellow- } \\
\text { green }\end{array}$ & $\begin{array}{l}\text { Very small } \\
\text { amount of blue }\end{array}$ & $\begin{array}{l}\text { Only combination tested using an } \\
\text { intermediary colour }\end{array}$ & \multirow[t]{3}{*}{ Low } \\
\hline $42 \%$ & Red \& Orange & No Blue & \multirow[t]{2}{*}{ No blue in either component colours } & \\
\hline $40 \%$ & Yellow \& Orange & No Blue & & \\
\hline
\end{tabular}




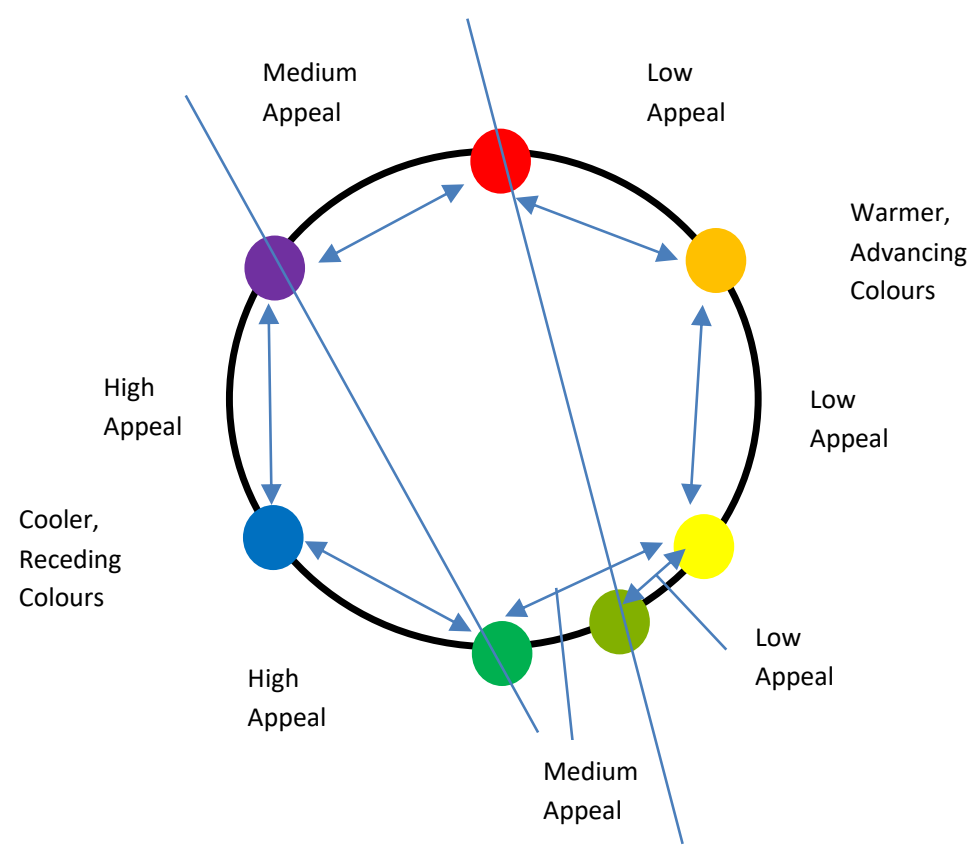

Figure 11. Colour wheel illustration of McLuckie's unpublished data relating to Table 1 (Source: Author's own interpretation)

Figure 11 shows the cooler receding colours in the colour combinations tested as having the highest appeal, followed by the more temperate colours; whereas colour combinations containing two warm colours are the least appealing. Blue and green, and in particular blue and violet may have been found to be most appealing as blue is generally a highly favoured colour in the UK. In addition, those samples are cooler colours that are also considered to be more favoured by western populations. Incidentally, Faber Birren had found that blue was consistently favoured in home products and consumer goods between 1954 and 1964, even during the trend for deep and muted colours in 1954, which steadily declined until considered unfavourable ten years later, by which time bright and clear colours had gained favour [323]. Birren, author of Colour Psychology and Colour Therapy (1979) was a strong advocate of colour affecting our emotions [324].

\subsubsection{Complementary colour schemes}

Complementary colour schemes are created using colours that are opposite each other on the colour wheel and therefore have no colour relationship. Harmony is said to be created through contrast rather than through colour relationship. According to Klarén and Anter [325] when complementary colours are placed next to each other they influence each other by increasing their respective 'chromaticness' (the term 'chromaticity' would be preferable) which creates a sense of harmony. Optimal contrast is created using the six-hue colour wheel, as the colour pairs will always comprise of one primary and one secondary colour. When using the twelve-hue colour wheel intermediary colours have an intermediary colour complementary and will always have a common colour relationship, though the appearance of this may not be so obvious to the untrained eye. For example, red-orange and its complementary bluegreen both contain an amount of yellow. There are a number of variations of complementary colour schemes as described below. 
According to the Swiss painter Johannes Itten [326], the eye requires a colour's complementary colour, and if not provided the human brain will spontaneously generate it. This common type of afterimage is known as successive contrast. However, in terms of consumer appeal, when using complementary colour combinations, the prospect of harmony being appealing in the fashion context may be contested. McLuckie [327] also conducted an experiment to test the appeal of complementary pairs in knitted samples using the same knit design. Thirty respondents rated the appeal of the knit samples using a 5point Likert scale, as previously described above. The results are given in Table 2 and visually in Figure 12. The red and green sample scored $58.6 \%$, the orange and blue sample had a lower appeal of $49.3 \%$, whereas the yellow and violet sample was least appealing scoring $46.2 \%$. The red-orange and green-blue samples were slightly less appealing than the red and green sample, yielding a score of $55.3 \%$. Similarly, the orange-yellow and blue-violet samples scored less than the orange and blue sample at $40 \%$. Surprising though, the yellow-green and violet-red samples scored $70.6 \%$.

Table 2. McLuckie's 1998 colour preference test results plus additional analysis relating to Figure 12

\begin{tabular}{|c|l|c|}
\hline $\begin{array}{c}\text { \% of } \\
\text { appeal }\end{array}$ & $\begin{array}{l}\text { Colours in the combination and number on the colour wheel opposite - The } \\
\text { numbers in brackets relate to the sample numbers also given in Figure 12 below }\end{array}$ & Acceptance \\
\hline $70.6 \%$ & Yellow-green \& Red-violet (1) & High \\
\hline $58.6 \%$ & Red \& Green (2) & Middle \\
\hline $55.3 \%$ & Red-orange \& blue-green (3) & Low \\
\hline $49.3 \%$ & Blue \& Orange (4) & \\
\hline $46.2 \%$ & Yellow \& Violet (5) & \\
\hline $40 \%$ & Yellow-orange \& Blue-violet (6) & \\
\hline
\end{tabular}

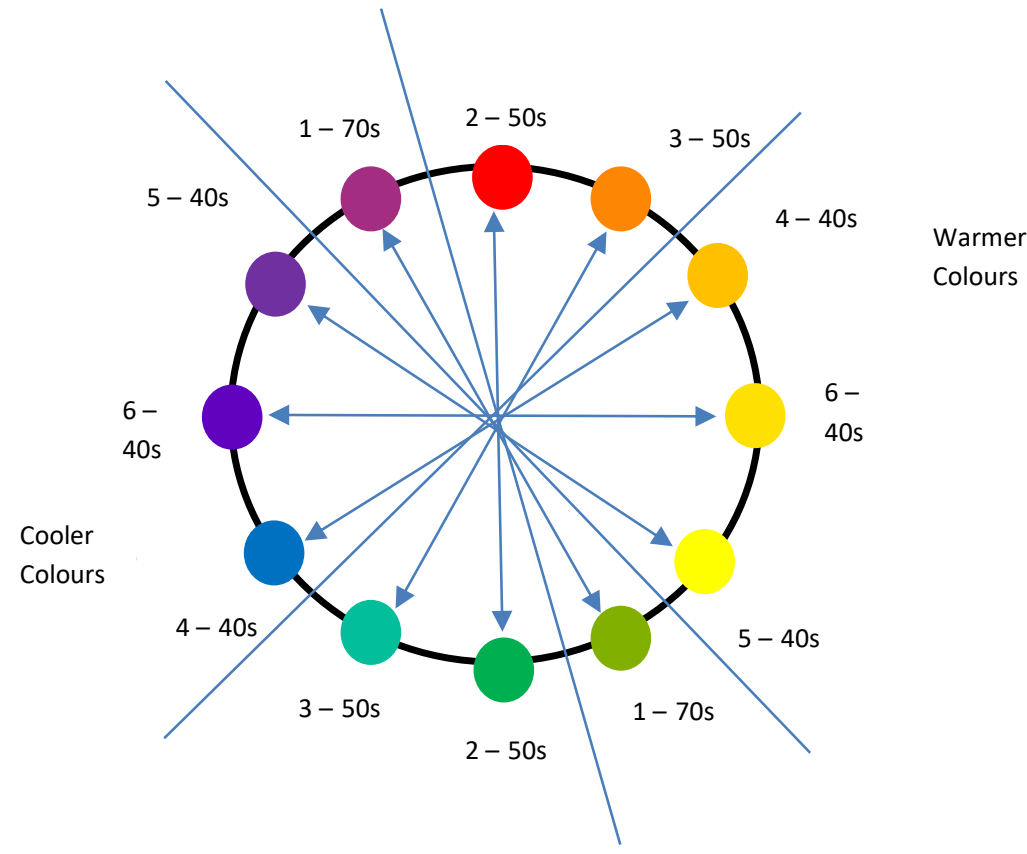

Figure 12. Colour wheel illustration of McLuckie's unpublished data (experiment two) relating to Table 2 (Source: Author's own interpretation) 
As can be seen in Figure 12 the pairing of warm colours with cool colours were least favoured, compared to the more temperate colours when paired. There is also a higher preference of blue hues when paired analogously than when paired with the complementary colours.

The split complementary colour scheme is a triad created by selecting one colour on the colour wheel and the two colours that sit either side of its complementary colour. For example, when selecting red, its complementary colour is green, blue-green and yellow-green sit either side of green on the colour wheel, the triad is therefore red, blue-green and yellow-green. On the six-hue colour wheel there are only two possible split complementary combinations available, the first contains red, blue and yellow, i.e. the primary colours, the second contains orange, green and violet, i.e. the secondary colours. When using the twelve-hue colour wheel there will always be a common colour relationship between two of the colours in the combination. The colour relationship in the above example, red, blue-green and yellow-green is green (or blue and yellow) in the latter two colours.

The double-split complementary colour scheme is a variation of the split complementary colour scheme using a tetrad of colours. In theory, two complementary colours are selected and the two colours either side of each on the colour wheel are used. For example, using the example given above, for the split complementary, red-orange and red-violet are used with blue-green and yellow-green, as they sit either side of red. In practice an oblong can be drawn on the inside of the colour wheel, the four colours will be indicated at each corner of the oblong. A further double split complementary colour scheme uses complementary pairs of equal distance on the colour wheel. Using a twelve-hue colour wheel every third colour is selected. Alternatively, a square can be drawn on the inside of the colour wheel. Due to the number of colours in the six-hue colour wheel this scheme can only be created on the twelve-hue colour wheel. It is also possible to use a trapezoid shape in the centre of the twelve-hue colour wheel. In theory this employs two split complementaries of a colour, plus the split complementaries of one of those split complementary colours. An alternative method is to select a colour, then select the second colour along the colour wheel, then select the next third colour, then select the next fourth colour, two colours will be left between the first and last selected colours.

\subsubsection{Discordant colour scheme}

In contrast to harmonious colour combinations, it is considered that while discordant is in effect nonharmonious in nature that the discord may still be pleasant or appealing because of the conflict against harmony. This is otherwise known as a clash combination. In theory it is another form of a split complementary but using only one of the split complementary pairs. The discord is greater when using the six-hue colour wheel. Another form of discord is to change the natural order of the colours. For example, if using orange and blue, orange is naturally a lighter colour than blue. By adding black to the orange to create a brown and adding white to the blue to create a much lighter blue, then the natural order has changed and the resulting pale blue with brown is a discordant colour scheme. Whether or not this combination is appealing or not is a matter of taste, possibly regardless of it being promoted as being on-trend or not.

The results of McLuckie's (1998) experiment to test the appeal of discordant pairs in knitted samples using the same knit design are given in Table 3. Again, the thirty respondents rated the appeal of the 
knit samples using a 5-point Likert scale. Eighteen combinations were tested. The results were as follows: blue and red-orange $73.7 \%$; blue and red $65.3 \%$; violet and yellow-green $64.6 \%$; red and blue green $62.6 \%$; green and violet also 62.6\%; green and red-violet $61.3 \%$; red and yellow-green $55.3 \%$; green and red-orange 53.3\%; yellow and red-violet 52\%; orange and green also 52\%; orange and violet 48.6\%; orange and blue-green 46\%; blue and yellow-green $45.3 \%$; blue violet and yellow also $45.3 \%$; blue and yellow $44.6 \%$; orange and blue-violet $42 \%$; yellow-orange and violet $40 \%$; yellow and red $39.3 \%$.

Table 3. McLuckie's 1998 colour preference test results plus additional analysis relating to Figures 13, 14 and 15.

\begin{tabular}{|c|c|c|c|}
\hline Appeal & Colour & Colour & Acceptance \\
\hline $73.7 \%$ & Blue & Red-Orange & \multirow{6}{*}{$\begin{array}{c}\text { High } \\
\text { See Figure } 12\end{array}$} \\
\hline $65.3 \%$ & Blue & Red & \\
\hline $64.6 \%$ & Violet & Yellow-Green & \\
\hline $62.6 \%$ & Red & Blue-Green & \\
\hline $62.6 \%$ & Green & Violet & \\
\hline $61.3 \%$ & Green & Red-Violet & \\
\hline $55.3 \%$ & Red & Yellow-Green & \multirow{4}{*}{$\begin{array}{c}\text { Medium } \\
\text { See Figure } 13\end{array}$} \\
\hline $53.3 \%$ & Green & Red-Orange & \\
\hline $52 \%$ & Yellow & Red-Violet & \\
\hline $52 \%$ & Orange & Green & \\
\hline $48.6 \%$ & Orange & Violet & \multirow{8}{*}{$\begin{array}{c}\text { Low } \\
\text { See Figure } 14\end{array}$} \\
\hline $46 \%$ & Orange & Blue-Green & \\
\hline $45.3 \%$ & Blue & Yellow-Green & \\
\hline $45.3 \%$ & Yellow & Blue-Violet & \\
\hline $44.6 \%$ & Blue & Yellow & \\
\hline $42 \%$ & Orange & Blue-Violet & \\
\hline $40 \%$ & Yellow-Orange & Violet & \\
\hline $39.3 \%$ & Yellow & Red & \\
\hline
\end{tabular}

The results are also shown in Figures 13, 14, and 15, to further highlight the colour combinations in relation to high, medium and low appeal respectively.

As can be seen in Figure 13 below, the colour combinations with the highest levels of appeal pair cooler colours with temperate colours or are two temperate colours. Each pair has a dominance of blue even if it is underlying within the colour composition, as opposed to being a dominant primary colour; for example, red-violet and green which both contain blue. In the case where the colours contain no blue, as in the case with red and red-orange, they are paired with blue or with blue-green. These colour combinations also have no yellow dominance. 


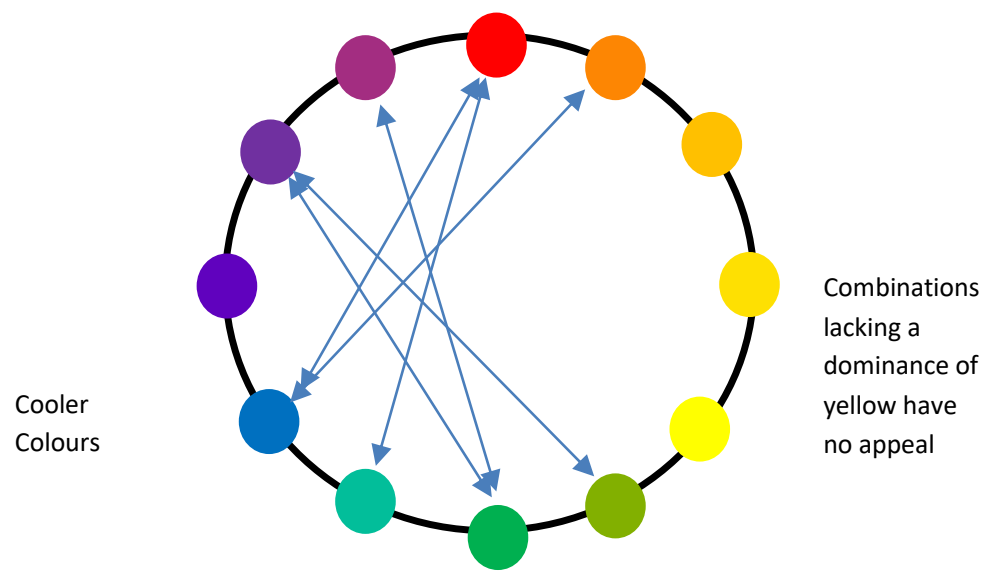

Figure 13. Discordant colour combinations with high appeal (Source: Author's own interpretation).

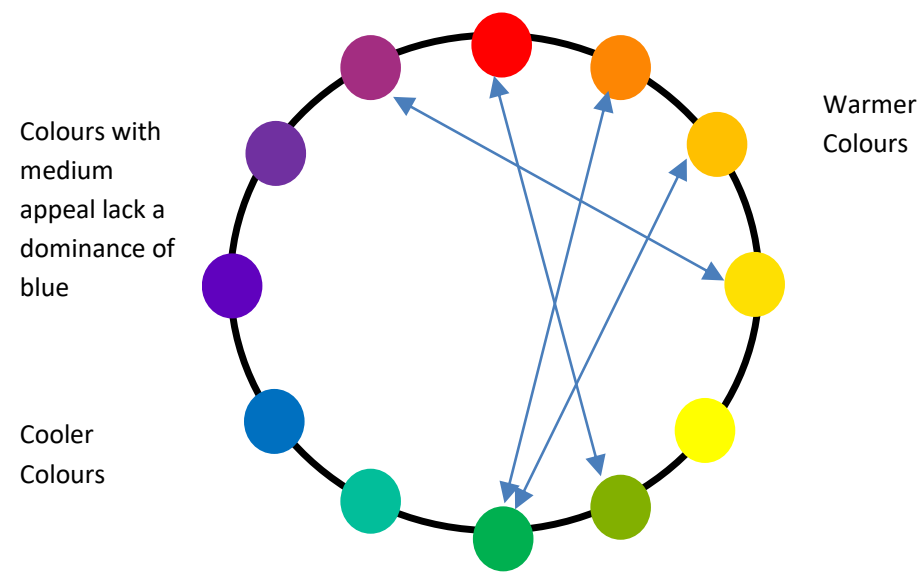

Figure 14. Discordant colour combinations with medium appeal (Source: Author's own interpretation).

As can be seen in Figure 14 above, the colour combinations with a medium level of appeal pair warmer colours with temperate colours. While orange and yellow-orange and their respective pairs appear in this group, yellow does not.

As can be seen in Figure 15 below, colours with dominant yellow, regardless of its pairing yield low levels of appeal even though blues are favoured colours in general, though when paired with yellow they have less appeal. 


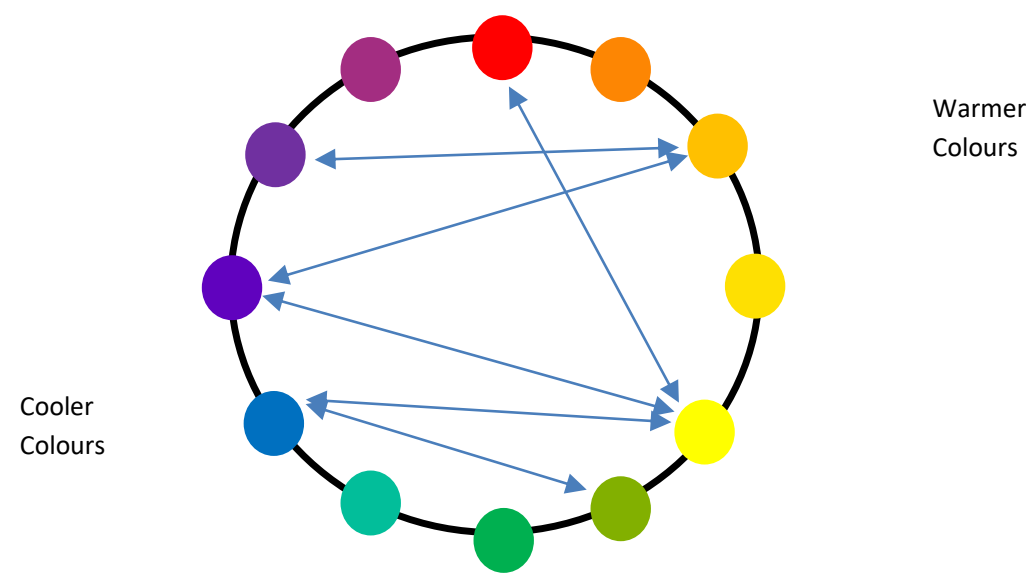

Figure 15. Discordant colour combinations with low appeal (Source: Author's own interpretation).

Table 4 shows all of McLuckie's results in order of appeal, some further interesting points can be deduced. The colour composition is calculated according to the composition of the primary colour within each colour pairing. Given that primary colours are $100 \%$ one hue, secondary colours are $50 \%$ of one hue and $50 \%$ of another hue, and intermediary colours are $75 \%$ of one hue and $25 \%$ of another, the two quantities for the two component colours in the pairing are now added together to give a total of $200 \%$ ( $100 \%$ for each component colour). In the first instance, looking at the colour combinations that have yielded an appeal score of $60 \%$ and above, five of the nine colours have blue as the dominant colour, that is, $100 \%$ or $150 \%$.

Table 4. McLuckie's 1998 results in order of appeal

\begin{tabular}{|l|l|l|l|l|l|l|}
\hline Appeal & Colour & Colour & Colour Scheme & \multicolumn{3}{|l|}{ Colour Composition } \\
& & & & \multicolumn{2}{l|}{ Red } & Yellow \\
\hline $73.7 \%$ & Blue (P) & Red-Orange (T) & Discordant & $75 \%$ & $25 \%$ & $100 \%$ \\
\hline $73.3 \%$ & Blue (P) & Violet (S) & Analogous (6-hue wheel) & $50 \%$ & 0 & $150 \%$ \\
\hline $70.6 \%$ & Yellow-Green (T) & Red-Violet (T) & Complementary & $75 \%$ & $75 \%$ & $50 \%$ \\
\hline $65.3 \%$ & Blue (P) & Red (P) & Discordant & $100 \%$ & 0 & $100 \%$ \\
\hline $64.6 \%$ & Violet (S) & Yellow-Green (T) & Discordant & $50 \%$ & $75 \%$ & $75 \%$ \\
\hline $62.6 \%$ & Red (P) & Blue-Green (T) & Discordant & $100 \%$ & $25 \%$ & $75 \%$ \\
\hline $62.6 \%$ & Green (S) & Violet (S) & Discordant & $50 \%$ & $50 \%$ & $100 \%$ \\
\hline $61.3 \%$ & Green (S) & Red-Violet (T) & Discordant & $75 \%$ & $50 \%$ & $75 \%$ \\
\hline $60.6 \%$ & Blue (P) & Green (S) & Analogous (6-hue wheel) & 0 & $50 \%$ & $150 \%$ \\
\hline $58.6 \%$ & Red (P) & Green (S) & Complementary & $100 \%$ & $50 \%$ & $50 \%$ \\
\hline $57.3 \%$ & Red (P) & Red-Violet (T) & Analogous & $175 \%$ & 0 & $25 \%$ \\
\hline $56 \%$ & Yellow (P) & Green (S) & Analogous (6-hue wheel) & 0 & $150 \%$ & $50 \%$ \\
\hline $55.3 \%$ & Red (P) & Yellow-Green (T) & Discordant & $100 \%$ & $75 \%$ & $25 \%$ \\
\hline $55.3 \%$ & Red-Orange (T) & Green-Blue (T) & Complementary & $75 \%$ & $50 \%$ & $75 \%$ \\
\hline $53.3 \%$ & Green (S) & Red-Orange (T) & Discordant & $75 \%$ & $75 \%$ & $50 \%$ \\
\hline $52 \%$ & Yellow (P) & Red-Violet (T) & Discordant & $75 \%$ & $100 \%$ & $25 \%$ \\
\hline
\end{tabular}




\begin{tabular}{|l|l|l|l|l|l|l|}
\hline $52 \%$ & Orange (S) & Green (S) & Discordant & $50 \%$ & $100 \%$ & $50 \%$ \\
\hline $50.6 \%$ & Red (P) & Violet (S) & Analogous (6-hue wheel) & $150 \%$ & 0 & $50 \%$ \\
\hline $49.3 \%$ & Blue (P) & Orange (S) & Complementary & $50 \%$ & $50 \%$ & $100 \%$ \\
\hline $48.6 \%$ & Orange (S) & Violet (S) & Discordant & $100 \%$ & $50 \%$ & $50 \%$ \\
\hline $46.2 \%$ & Yellow (P) & Violet (S) & Complementary & $50 \%$ & $100 \%$ & $50 \%$ \\
\hline $46 \%$ & Orange (S) & Blue-Green (T) & Discordant & $50 \%$ & $100 \%$ & $50 \%$ \\
\hline $45.3 \%$ & Blue (P) & Yellow-Green (T) & Discordant & 0 & $75 \%$ & $125 \%$ \\
\hline $45.3 \%$ & Yellow (P) & Blue-Violet (T) & Discordant & $25 \%$ & $100 \%$ & $75 \%$ \\
\hline $44.6 \%$ & Blue (P) & Yellow (P) & Discordant & 0 & $100 \%$ & $100 \%$ \\
\hline $44 \%$ & Yellow (P) & Yellow-Green (T) & Analogous & 0 & $175 \%$ & $25 \%$ \\
\hline $42 \%$ & Orange (S) & Blue-Violet (T) & Discordant & $75 \%$ & $50 \%$ & $75 \%$ \\
\hline $42 \%$ & Red (P) & Orange (S) & Analogous (6-hue wheel) & $150 \%$ & $50 \%$ & 0 \\
\hline $40 \%$ & Yellow-Orange (T) & Violet (S) & Discordant & $75 \%$ & $75 \%$ & $50 \%$ \\
\hline $40 \%$ & Yellow (P) & Orange (S) & Analogous (6-hue wheel) & $50 \%$ & $150 \%$ & 0 \\
\hline $40 \%$ & Yellow-Orange (T) & Blue-Violet (T) & Complementary & $50 \%$ & $75 \%$ & $75 \%$ \\
\hline $39.3 \%$ & Yellow (P) & Red (P) & Discordant & $100 \%$ & $100 \%$ & 0 \\
\hline
\end{tabular}

As can be seen in the table, the colour schemes: complementary, analogous, and discordant are evenly distributed in terms of appeal. The colour scheme in itself does not appear to be of great importance but the composition of the colours is. Furthermore, if all of the compositions red, yellow, and blue are added together in their respective columns and in groups of high, medium and low appeal, where high appeal is $60 \%$ and above in the left hand column, medium appeal is $50 \%-59 \%$, and low appeal is $49 \%$ and below, as shown in Table 5 below, group one has the highest blue content, group two has the highest red content and group three has the highest yellow content. It may therefore be deduced that overall, colour combinations with a high blue composition may yield higher levels of consumer appeal, whereas those with a higher yellow composition may yield much lower levels of consumer appeal. Colour combinations with a higher red composition may yield medium levels of appeal, and may be even more subject to personal colour preference ratings. It should however be noted that the promoted trend colours at the time of McLuckie's experiments were not recorded. It should also be noted that this research was conducted in the UK, research to the same effect in different countries may highlight different cultural preferences. In addition, as the research was conducted in the Midlands, there may even be some interesting regional differences if tested across the UK.

Table 5. Groups (high, medium and low appeal) by primary colour composition.

\begin{tabular}{|l|l|l|l|}
\hline & Red & Yellow & Blue \\
\hline Group 1-60\% plus & 575 & 350 & 875 \\
\hline Group 2-50-59\% & 800 & 600 & 400 \\
\hline Group 3-49\% and below & 775 & 1,250 & 775 \\
\hline
\end{tabular}

It should also be noted that while consumer colour preferences may affect the judgement of rating samples testing the appeal of the colour combinations, and that blue has been shown to be a particularly influential colour, not all colour combinations containing blue were highly favoured. This would further suggest that the overall aesthetic which may involve colour shift phenomena should be of high consideration when putting colours together for colour palettes, colour forecasts, and colourways 
for product ranges. Another noteworthy point is that there is no recorded consideration of how the colour combinations relate to colours available to consumers at that time, where any of the tested colours may be in the fashion cycle (see Section 7), nor how the tested colour combinations relate to the zeitgeist (see Section 3.5). No study to date has been identified that takes all of these factors into consideration when researching colour preferences, for consumer products or otherwise. What can be deduced is that having a good understanding of colour schemes may not give absolute guarantees but can help forecasters to produce appealing colour combinations.

\subsection{Colour as a powerful sales tool}

The role that colour plays in the consumer purchase decision process cannot be underestimated and therefore neither can the importance of colour as a powerful sales tool. According to Agrawal and Singh, a minimum of $62 \%$ of purchases made are based on the colour of the product and in some cases this may be closer to $90 \%$ [328]; though indications of the different product types, either tested or made reference to, were not disclosed. As Burns, Mullet and Bryant [329] note, that as consumers use colour as a tool for the selection of products, it is essential to understand colour preferences at given periods of time as tastes change over time. Keiser and Garner [330] propose that colour is reputed to be 'one of the first things that attracts a customer to a garment' because colour 'speaks to individuals on so many levels'. Danger [331] suggests that 'the first step towards using colour to better advantage may well be to audit colours and weed out those that have outlived their usefulness'. The second is to 'bring the colours into line with consumer desires and to relate them more satisfactorily to current market conditions'. Danger recommends that one should 'study the market and consumer trends in detail' and should ensure that 'every colour offered has a reason behind it and does something positive for sales'. Of course, colour forecasters claim to do all of this, though with the speed at which trends change today compared to at the time of Danger's writing, plus other complexities of a much more competitive marketplace today, how can colour forecasters feasibly undertake such a huge undertaking, particularly for those forecasters who operate much closer to the retail period. Danger goes on to say that terms such as 'fashion colours' suggest 'promotion themes that can be linked to colour'. Again, this is how forecasters claim to operate today as colour management has become more than a commercial means to control colour solely for the purpose of production, which is further discussed in Section 6.6.1.

In a practical sense, Keiser and Garner [332] briefly discuss how colour can be used by individuals to create 'figure illusions'. For example, based on colour theory, 'warm colours look larger as they are advancing, cool [colours appear] smaller, receding. Brights call attention and add bulk, [whereas] light colours are more flattering to the face'. In addition, 'colour harmonies also affect how we perceive the figure'. For example, 'monochrome [combinations] create illusions of height; though they further note that not all 'customer groups' are 'sensitive to these nuances'. For the benefit of the consumer, Keiser and Garner suggest that 'understanding how colour affects the figure can help to provide options when selecting a colour range for a particular silhouette or when determining how best to use colour harmonies that are in fashion', yet consumers are not typically given the knowledge and tools to do this for themselves, and for those who have can feel very frustrated when their colours are not in fashion and therefore unavailable to them. For example, Mary Spillane, founder of the colour consultancy agency Color Me Beautiful, is a devout advocate to the importance of colour in fashion and personal 
image. When interviewed for Drapers Record [333] to promote colour analysis as a selling tool for fashion retailers, positive and negative responses were raised. A women's wear retailer was quoted as saying 'often personal colour analysis doesn't work well with particular fashion trends in colours'. Another retailer felt that image consultants create problems for retailers as potential customers are looking for certain colours that are not available due to current trends and added that 'most customers have a good idea of what shapes and colours suit them already'.

Furthermore, Keiser and Garner [334] state the necessity to relate colour palettes to target markets as the demand from consumers will be different, and notes demographic information to be critical, including 'age and life stage, fashion level, colouring, ethnic diversity, geography and climate [and the] garment's function'. Their reasons are given briefly as follows:

i) 'Age and life stage: target customers have unique [colour] preferences ... younger respond more to trends ... older develop a sense of dress code, practical, awareness of personal colouring and confidence

ii) Fashion level: couture and designer markets introduce new colours ... in any given market there are fashion forward consumers, a colour palette should accommodate these preferences fashion-forward colours and mass-consumption colours (fashion / staple)

iii) Personal colouring: warm and cool colours

iv) Geographical location: weather patterns and regional preferences, in some parts of the world colours have strong cultural meanings (this is discussed further in Sections 8 and 9.3)

v) Garment's function or end use: Men's, Women's and Children's markets, plus type of garment, for example, swimwear, sportswear, outer-wear.'

Colour stories need to be managed. Within the retail and manufacturing sectors this management of colour moves beyond the more subjective manipulation of colour. For Puhalla [335], there are no rules, as in accordance with that research study, in order to find guiding principles of fact, 'it is a commonly accepted notion that it is best to keep colour combinations to a minimum number of components .... (as) the brain is able to understand and organise the information, ... and multiple colours create visual noise making it impossible to focus on anything', which gives good reasoning for limiting colour within palettes at any one period of time.

\subsubsection{Colour management, measurement and tools}

As Keiser and Garner [336] state, 'seasonal colours are critical to meeting profit objectives' for fashionrelated product industries. A balance is required between what has been determined by forecasters as likely demand from consumers and the necessary efficiencies. Colour palettes and colourways for product ranges require sufficient choice for consumers to select from but not so many that may create confusion. In order to maintain the colour characteristics and quality of the individual colours in the palette strict control is necessary to produce the same colour on different fibre and fabric types; a process known as colour matching. Keiser and Garner also note that the way in which colours are used 'in combination in any given season' is just as important as 'selecting trend-right colours for a particular 
target market' and make reference to schemes such as harmonies, monochromatic, analogous and complementary combinations (discussed in Section 6.5).

Colour technologists and scientists prefer to use both objective and subjective measurement of colour whereas designers and forecasters prefer to use subjective visual assessment. Colour technologists interpret the designers colour choices either through colour measurement and making adjustments as appropriate on the designers' recommendations, again through interpretation until the desired colours are achieved, or, and more commonly, by using systems such as Pantone's colour standards as the colour-communication tool between the two sets of professionals. However, colour measurement is a preferred method of colour identification in colour-forecast information in order to enable designers to accurately reproduce the trend colours as promoted. Pantone's colour-coding system is widely adopted by the textile and fashion industry. They provide a range of tools for colour measurement and colour matching. Their colour code system comprises colour-chip colour guides using RGB values (Red, Green, Blue - additive primary colour mixing principles). Most of the colour-forecasting materials and packages also used the Pantone colour reference system. A range of colour-measuring instruments or spectrometers are available for the accurate measurement of colour composition enabling colours to be fully described for the purpose of repeatability, or colour matching. Hand-held devices are useful tools for forecasters, designers, product developers, and other colour specialists because colours can then be measured by their absorption or reflectance and recorded during research field trips. Spectrometers are the more-sophisticated instruments that measure the amount of light at each wavelength; they provide the most-accurate colour measurement and are capable of detecting metameric effects. By contrast, colorimeters emulate the human eye [337]; this type of instrument cannot detect metameric effects. Pantone supply a useful hand-held colorimeter device that forecasters and designers can use on location to record fairly accurate colour measurements for their field notes.

The American Association of Textile Chemists and Colourists (AATCC) publishes colour standards for both visual and instrumental colour measurement as a tool for objective measurement and colour management. For colour matching purposes descriptive procedures and conditions for undertaking tests are given. The need for creating order through colour systems, according to Boeri [338], goes beyond the commerciality of product as being our 'innate desire to create order', a need from which the concept of universal charts and systems originated. Colourists can learn a lot from such systems and the underlying philosophies for their colour work and colour management preferences. Wilhem Oswald's system, for example was based on the 'perceptual comparison of respective similarities and differences among colours. Latvian chemist Oswald (1853-1932), studied colour for most of his life in order to produce a scientific standardising system [339]. Oswald's system employed a 24-hue colour wheel with red, yellow, blue and seagreen plus secondary principle colours orange, purple, turquoise and leafgreen'. References to particular colour names is particularly interesting as it not only links to colour naming used today in the fashion industry and in particular to colour and trend forecasting, but also to find expression for different green's through the use of language which does not in itself provide an adequate universal interpretation of colour. According to Puhalla [340], 'colour is an intrinsic visual attribute of form ....... and perception $\{i$ is a\} strongly emotional part of the visual process', yet colour can be arranged in accordance with 'objective codes', which is 'critical to making information understandable'. Furthermore, Puhalla states that 'colour can be formulated objectively leading to visual order of importance by controlling the visual relationships of hue, value and chroma contrast ... to 
assist our perception of importance and dominance within a prescribed colour combination, ... and form a colour language and structured patterns of colour organisation can emerge'. As Mottram and Jefferies [341] state, colour specifications for a global industry, that requires 'specificity and exactitude' can be particularly problematic for designers, and presumably also for colour forecasters. Therefore, a number of systems have emerged as useful tools including, in chronological order (according to Mottram and Jefferies):

i) Munsell's system, which is used extensively in the US;

ii) The Pantone Colour System;

iii) The Natural Colour System (NCS);

iv) The Commission Internationale de l'Eclairage (CIE) (International Commission on Illumination) - through the Division of Vision and Colour, the organisation studies 'visual responses to light and to establish standards of response functions, models and procedures of specification relevant to photometry, colorimetry, colour rendering, visual performance and visual assessment of light and lighting' [342]; and

v) The British Standards for Colour for the building and decorative paint industry [343].

Aforementioned American painter Albert Henry Munsell developed his system around the three colour characteristics hue, value (lightness) and chroma (saturation) and five primary colours, red, yellow, green, blue and purple. The Munsell Color Order System has hues around the 'equator' of a sphere, which are number coded in relation to 5 on the value scale. The vertical column within the centre of the sphere represents the different tonal values. Each chromatic hue is arranged on a wing, or 'horizontal slice' having varying intensity. The various hues therefore encompass the circumference of the sphere. The sphere is not uniform as Munsell had recognised that not all colours require the same number of steps of chroma from fully saturated to grey; more steps are required for lighter colours than for darker colours. A textile-related variation of the Munsell System, SCOTDIC, with three sets of samples (glossy on polyester crepe, matt - on cotton poplin and yarn - wool yarn) was created by the marriage of two different approaches, The Standard Color of Textile (a Japanese system) and Dictionnaire Internationale de la Couleur (French). It is widely used.

Pantone claim to have developed their system for the purpose of communicating colour. This system uses a 'universal colour language' and a 'unique' coding system [344]. Creative Director of Gather Journal, Michele Outland when interviewed by Budds [345], declared the Pantone Colour System to the standard for the industry. According to Keiser and Garner [346] the system employs a six-digit code where the first two digits relate to the value - 0 being light and 19 dark, the second two relate to the hue - numbered 01 which is Yellow through to Yellow-Green numbered 64. The last two digits relate to chroma - neutral grey is numbered 00 through to maximum hue strength numbered 64 . Pantone also note that a suffix is used for each colour code, TC is used for colour swatches where the colour is dyed on cotton fabric. TPX is used for colours printed onto paper [347].

The colour wheel is a basic tool predominantly used to understand colour mixing and colour relationships when working with pigments such as paint, but not all colour theorists have agreed with this basic tool being sufficient. For example, Ewald Hering relates his colour theory to six primary 
colours: the three basic colours he selected for subtractive colour mixing namely red, yellow and blue, plus the secondary colour green, plus black and white; the Swedish Natural Colour System (NCS) is based on this theory. The NCS is said to 'describe colour perception' in relation to hue, nuance and chroma. Hue is described as being 'a relationship between two chromatic elementary (primary) colours'; Nuance is described as being 'a relationship between black, white, and the most chromatic of each hue'. The NCS colour wheel comprises only four of the elementary (primary) colours, red, yellow, blue and green, but have nine distinctive variations of each. The four hues are plotted on the wheel and labelled as $\mathrm{R} 10 \mathrm{~B}$, which is located next to red, through to R90B, which is located next to blue; also located next to blue is B10G through to B90G which is located next to green; also next to green is G10Y through to G90Y which sits next to yellow; finally, also next to yellow is Y10R through to Y90R located next to red. The colour nuances are then plotted for each colour variation on separate triangle-shaped grids with variances of added black and added white of two sides of the triangle approaching the hue in question, and variances of grey plotted between white and black on opposite corners of the triangle with the gradations of colour nuance plotted appropriately on the grid [348].

\section{Colour theory models for colour forecasting}

According to Tate [349], in the colour selection process, the development of themes, colour stories and colour palettes (Section 3.2,) ideally colour palettes should contain fashion colours, staple colours, warm and cool colours, neutrals and dark colours, and as stated in Section 5.1, a sound and, preferably, extensive understanding of colour and colour theories will serve the forecaster well. A range of theoretical models exist to aid the forecaster in developing what may be called, balanced colour forecasts. While authors present these as theoretical concepts, no evidence can be found to show that such tools are exploited by forecasters or applied in practice.

One such model based on the four seasons is a typical approach used by colour consultants and many personal colour and style agencies for example the aforementioned 'Color me Beautiful' image consultancy founded in America and brought to Europe and the UK in the early 1980's [350]. Many similar companies exist, all of which have devised their own variations of the basic seasonal colour model, such as Barbara Jacques in the UK [351]. The consultancy group train individuals to be colour consultants or colour and style consultants to advise a growing network of clients. This type of business has more-recently expanded into the retail sector where they are known as personal shoppers.

According to Rath, Petrizzi and Gill [352] personal shoppers are 'salespeople who devote their time to meeting the fashion needs of select customers, referred to as clients, by supplying fashion information and choosing merchandise tailored to them in their business and social lives'. They inform their clients of newly-available merchandise in key stores. Some retailers employ personal-shoppers in-store to offer a personalised service, such as Bloomingdale's, Macy's and Selfridges. However, there is no real clarity in academic literature about how such individuals are trained and their expertise in colour, styling, or trend knowledge.

Brannon [353] provides the four season's model as a useful colour forecasting tool. This basic model takes form through the natural perceptions of each season (see colour bias, Section 6.4.2.1): 
Winter being cold and crisp where we often experience contrasts of colour through brightness or intensity. Winter colouring is therefore described as having cool undertones (blue bias) and are clear, bright and intense.

Spring colours retain the clarity, brightness and intensity of the winter colours, but as springtime is known for its rise in temperature, relative to the winter months, the colours are warm (red and yellow bias).

Summer colours are in contrast to the spring colours having cooler undertones and are less clear, i.e. subdued, and less intense.

Autumn colours retain the subdued lesser intensity of the summer colours but have warmth. The clarity and intensity of colour changes as we return to the cool winter colours.

The rationale behind the four seasons model is that an individual's personal colouring (hair, eyes, skin tone) will align more to one of the four season types. In reality, this would not actually appear to be the case as Spillane (1994) and Jacques (1994) have developed their own extended model variations. The Nippon Color \& Design Research Institute patented another model in the early 1990's. This 3D model also used colour temperature (warm and cool), colour value (clear and greyish) and intensity being described as hard or soft [354]. While they repute its value as a colour forecasting tool it would appear that its primary function was to analyse sets of colours, probably existing forecasts rather than as a means to anticipate the direction of colour choice for the near future.

Contrary to common belief, single colours tend not to be the primary focus of a forecast. Keiser and Garner [355], when referring to the colour associations CAUS, CMG and ICA, state 'their colour palettes project the course that colours are likely to take - warmer or cooler, lighter or darker, clearer or greyer (hue, value, saturation) [as well as] the relative importance of the hue'. Levenbach \& Cleary [356] describe the act of forecasting as using techniques and tools that are applied in a systematic manner. Such techniques include analysing and interpreting the drivers of the trend, i.e. the factors that motivate trends to happen. Part of the forecasting process is to explain what those motivations are, where they are moving the trend to, and why this is happening, in order to justify why this 'prediction is likely to occur' [357]. Part of this analysis involves tracking the movement of the trend. To do this forecasters estimate 'the probable acceptance' and by 'calculating the time [it] will take to move to mainstream [358]. One particular method used to track the movement of a trend is through the trend's evolution patterns and direction. The following Subsections build on the subjective approaches of the colour forecasting process previously discussed in Section 3, the colour selection processes described in Section 3.2, the initial overview of direction and speed of change in Section 3.4, and takes into consideration the observation skills of the forecaster presented and clarified in Section 5.2.1.

\subsection{Colour evolution}

The traditional colour-forecasting process is evolutionary, just like fashion, moving on a seasonal basis via the forecasters' seasonal colour palettes. This evolutionary process is followed once the colour or 
colour story has been identified. Keiser and Garner [359] propose that forecasters will seek answers to the following series of questions as part of the tracking process:

vi) Was the colour in the market last season?

vii) How saturated was it?

viii) Was the colour story based on clear primary colours or offbeat, unusual ones?

ix) How should the colour story be developed for next season?

Keiser and Garner further propose that colour themes 'help the consumer to understand the importance of new colours' though how this is so, is not elaborated on and it could be questioned which sets of consumers are likely to notice given that not all of the population have an interest in following fashion trends intently, if at all. McAssey and Buckley [360] note that forecasters generally create a narrative that explains how the colours are evolving from one trend into another. However, through their example it is suggested that different trends respond differently within the same time frame: 'perhaps one is becoming more muted whereas another is getting brighter, or one colour is becoming closer to another, or one is getting darker where another lightens up'. While this is put into the context of the forecaster narrating the key changes of the trend from the previous season, it does appear that there is a suggestion of the necessity to track individual colours, and not to assume that one season favours bright colours where the following season favours muted colours, i.e. palettes of colours are not to be tracked, but individual colours, which presumably must then be repackaged into new colour palettes.

According to Rousso [361], the movements of fashion and trends occur through a range of courses, they can 'flow, swing, curve and repeat'. In order to analyse and determine the course of a colour trend the tools outlined in the following Subsections can be used.

\subsubsection{The fashion pendulum}

Brannon [362] refers to the fashion pendulum as a visual aid to demonstrate the swinging movement of a fashion trend. This is likened to the pendulum of a clock, where the two extremities are where the pendulum has reached maximum momentum in one direction forcing it to swing back to the extremity on the other side of its swing. This tool can be used in a number of ways for colour from observing the gradual change of a hue, its intensity or its value. For example, a red may begin as a red-orange pillar box red at one extreme and gradually move towards the colour of a red wine with a gradual addition of black; or the undertone may change from yellow to blue creating a cooler burgundy shade. The forecaster would need to have mastered their understanding of colour mixing theory in order to observe the subtle changes in colour, or would otherwise need to measure colours accurately to understand the colour shifts. Rousso and Ostroff [363] state that the swing can be very slow or extremely rapid and that a new trend will begin that is the opposite to the first trend. There are no academic research studies of the movement of colour change, so it is not known whether it is as simple as tracking only the change of the hue, or only the change of its intensity or possibly requiring more-complex analytical procedures and how well, or to what extent, it would be done by forecasters. Research that can evaluate correlations 
between the swings in an individual's colour taste, the colour zeitgeist and more general colour preferences may provide a valuable key for forecasting colour for target-market-specific products.

\subsection{Diffusion, diffusion curves and the fashion cycle}

Sproles [364] proposed that the measurement of diffusion through the use of diffusion curves to be useful for forecasters. Diffusion curves track adopters of the trend over periods of time. The rate of acceptance is said to be analysed and an evaluation of current acceptance realised. With this knowledge, it should be possible to estimate the likely extent of the trend. Forecasters usually understand the current acceptance of a trend through their observations and as part of their intuitive processes. Diffusion relates to innovation and also relates directly to timeframes which are given particular titles, such as fads and classics, which are discussed in Sections 7.2 and 7.4.

The 'Diffusion of Innovation' theory, formulated in 1962, is reputed to be used by trend forecasters [365]. Rogers [366] had observed that whatever the trend, and regardless of who initiated it, movement through groups of people, communities and / or societies always follows the same pattern, i.e. from an innovator, through different types of adopters, beginning with those with the least resistance to change, and finally to the laggards, those with the most resistance to change. The innovation itself can be a new fashion look.

The fashion cycle has been developed from Rogers' diffusion of innovation theory in order to 'follow acceptance and rejection' of trends as it can be used to track the movement, i.e. the direction that the trend has taken, and is taking, and also the speed at which the trend is moving. This is normally expressed visually as a bell curve. While many authors show the curve as a uniform shape, literally like the shape of a traditional bell for the purpose of demonstration, in reality the shape will be determined by the movement of the trend. Marketers also use this type of tracking tool for the product lifecycle to track the sales of a product, or product line. Some retailers may use it to track colour trends though this cannot be verified at this time in literature. The axis on the product lifecycle model and of the fashion cycle measures sales over time, the vertical line charts sales or customers and the horizontal line determines the timeframe. The curve shows the sales and the profile line moving through the introduction, growth, maturity and decline stages [367].

In theory, the trend moves through sets of consumers in turn from those who take up the new trend very quickly through to those who engage with the trend much later, though there are some who will never engage with that particular trend. The theory is discussed at length in the following Section 7.2.1. However, it is noteworthy to state here that some trends may be taken up more naturally by some than by others who respond to the marketing and branding activities of the retailer. It should also be noted that not all trends are adopted by all people, and therefore trends can also be considered as niche. Holland and Jones [368] remark that forecasters will identify the trend when it is in the earliest stages of adoption, this may suggest one of two things: 1 ) the trend is one that was previously directed by the forecasters and is now becoming visible on the street for forecasters to track, or 2) the trend has emerged without the authority of the forecasters. 
Keiser and Garner [369] provide the following explanation of the five stages of the fashion cycle bell curve in relation to the product lifecycle and Rogers' diffusion of innovation theory (some analysis is also provided in relation to the work of Rousso [370] and Easey [371]):

1. 'At the introduction stage, new trends are introduced on designer runways, or put together by influential fashion innovators as part of grass-roots movements. These trends are worn by fashion innovators who have the means to buy designer fashions or who take the lead in putting together innovative street looks that send fashion in a new direction'.

Keiser and Garner recognise that not all trends are instigated by the fashion industry or directed only by forecasters. The industry moves trends deliberately through the product lifecycle using a strategy known as planned obsolescence, and therefore the curve can be forced to be uniform, or to plateau for an extended period of time to take advantage of more sales at a peak time. It would be very interesting to map trends directed by forecasters against trends that happen on the street as part of the grass-roots movement; this also links to the theories of adoption. For Rousso, the introductory stage 'begins with a visionary spark' and that the 'timing into the market is crucial - a fashion mood or idea appears on the horizon, acknowledged by innovators'. Keiser and Garner's and Rousso's interpretations of the introduction stage differ in that the former believes it to relate more to inspiration or visionary activities whereas the latter relates the stage to research activities.

2. 'During the growth stage, fashion leaders or early adopters purchase the fashion as it becomes more widely available at contemporary designer, bridge, and fast fashion contemporary price points'.

This is at the point where the trend often becomes modified to make it more palatable for different target markets. It is not clear how the variations of the trend are monitored. Rousso refers to this stage as 'Rise'. It is where the trend is 'recognised and copied [it is] accepted by more people, planning [is] initiated for mass market, [and] copied by manufacturers with cost of reproduction reducing; increased volume at reduced cost'.

3. 'During the acceleration stage the trend is interpreted and widely available at a mainstream level and worn by fashion followers' (early majority mostly, and some late majority).

It is at this point where retailers choose whether or not to re-stock to extend the potential life time of the trend while it still has demand from consumers. However, retailers in the past have found this to be a costly mistake if they invest further in the trend and misinterpreted the likely ongoing demand. This strategy results in a lot of unsold stock unless sufficient sales can be made by the late majority. Perhaps to ensure that this risk is minimised retailers prefer to terminate investment forcing the trend to end, at least on the high street. In which case it is not difficult to understand why the industry has a preference for supporting shorter controlled trends in a given season and using this strategy within their branding efforts to market themselves as being fashion-forward. Easey infers to this to be part of the growth stage. For Rousso this is the 'Culmination' stage where the trend 'reaches its height of popularity, [it is] 
considered to be a major trend [as it is] accepted throughout multiple markets; [in] product development [it is when] new details [and] colours [are considered and the trend] may become a classic'. Rousso appears to group growth and saturation under the umbrella term culmination.

4. 'During the saturation stage, the fashion is at its height of popularity and is widely available to mass markets at all price points. Product developers at all levels should be on to the next trend'. At this point the late majority and some of the laggards will be engaging with the trend as its' popularity is highly visible. Easey refers to this as the maturity stage.

5. 'During the decline and obsolescence stage, consumers may continue to wear the fashion, but they are no longer interested in purchasing additional items unless it is at greatly reduced prices. Eventually, the fashion item looks dated and is impossible to sell'.

While it might be true that consumers no longer wish to purchase more of the same, this is the critical point of where products enter into a life-phase that is not currently captured in the product lifecycle management process as it is beyond the retailers' control, nor is it captured in the trend tracking process. This is where products are discarded (literally thrown away), kept and worn for extended periods of time and may become a cherished item, or move into the recycle process. This stage has attracted a great deal of interest and research in relation to the closed loop, or circular economy. WRAP, the Waste Resources Action Programme (a charitable body in the UK) for example, have initiated the Sustainable Clothing Action Plan (SCAP) to 'improve the sustainability of clothing and textiles across its lifecycle'. The design of clothing is recognised as a critical tool for the closed-loop economy to extend the attractiveness and durability of garments, thus increasing their longevity in the first cycle and to facilitate further multiple cycles before entering a lower-value recycling process [372]. Colour can be used to support the circular economy if a system-change is made to the current colour forecasting process and practice. The Ellen MacArthur Foundation, another UK-based charity, supports the need for change at the system-level for three-fold long-term benefits: the economy, the environment and societal wellbeing. In their report, A new textiles economy: Redesigning fashion's future, the vision for system-change emphasises the need for innovation to 'transform the way clothes are designed, sold and used' and to 'break-free of the increasing disposable nature' of fashion and the excessive consumption mind-set. Again the longevity of garments is valued as a move towards more sustainable practice and note that the 'average number of times a garment is worn before [discarding] has decreased by $36 \%$ compared to 15 years ago'. Their circular economy philosophy is one of 'restorative and regenerative by design'. In order to achieve this through increasing the utilisation of clothing, design should create 'high value during use', the ability to 're-enter the economy after use' and 'never end up in waste' [373]. In order to facilitate such change, a system-level change to the colour forecasting process is imperative.

Rousso describes the decline stage as one of 'oversaturation [where] repetition of the look [is evident and there is a] decline in interest and decreased demand from consumers, mark-downs and price incentives [can be seen on the high street]'. For Rousso decline and obsolescence (Section 7.2.2.) are two different stages. The obsolescence stage being where the 'lack of interest and no interest in the product, [is evident and there is] no retail potential at any price, consumers [are] reluctant to buy'. Davis [374] makes a noteworthy point, in that the more traditional bell curve that normally spanned a three to 
five-year period had been replaced at the time of his writing in the early 1990s by 'a plethora of microcycles, each oriented to a different identified segment of the apparel market'.... and as demonstrated through market research, 'there is not likely to be a single reigning fashion at any moment in time; thus there are cyclical irregularities within identifying segments as well as between them'. This emphasises that forecasters can no longer track every trend and has allowed the trend forecasting sector to develop and grow through their own segmentation and tailored forecast provision. However, the fashion cycle does not really account for taste and colour preferences, rather it indicates the volume of the public willing to engage with the trend, or strength of the trend in a given period of time and perhaps how quickly those participants began to lose favour of the trend, as opposed to allowing any kind of understanding of what a stronger trend may look like, or might have being. Yet, Keiser and Garner [375] propose that the fashion cycle is a 'very important tool for trend forecasters'. While the reasoning for this makes logical sense, 'equally important to recognising a new trend is anticipating when a fashion idea is in decline, since new products are planned anywhere from one month to nine months in advance of a season', there is little recognition that new tools could potentially provide benefits not previously experienced by forecasters, or that the current tools could be viewed as being somewhat out-dated. This point is expanded upon in Section 10.

\subsubsection{Diffusion theory}

Diffusion theory relates to how a trend is disseminated, or how it spreads. As noted in the previous Section, the theory is often linked to the general product lifecycle model, and to the fashion cycle both of which are discussed at more length in this Section. Knowledge and observations of adoption theories, the reasons for why a trend is popular, or not, the speed of the movement through the product lifecycle and how the trend is changing, which can be tracked through the pendulum tool, are all part of the forecasters' tool kit. According to Raymond [376] and Meadows [377], Roger's adoption 'types' are used by forecasters and that they are guided by his estimated percentages of diffusion in order to more accurately 'calculate the size of each group in any overall society or sector'. Holland and Jones [378] give those percentages as follows: Innovators (2.5\%), Early adopters (13.5\%), Early majority (34\%), Late majority (34\%) and Laggards (16\%). The types are per product type, there is no suggestion that an individual will be an innovator type for clothing, electronic devices and food, for example, and it is therefore accepted that a person who actively seeks new electronic devices may be a laggard where fashion is concerned, or vice versa. Also there is no reason to believe that a 'once an innovator always an innovator' rule applies, but it is presumed that at any one point in time, overall, for any product type, the guideline percentages will be relevant and accurate, and have therefore become a general rule of thumb.

Rogers' had also recommended a further model to support his diffusion of theory. This five-stage process is given by Meadows [379] as follows:

i) 'Knowledge - learning about the existence and function of the innovation' (It is assumed that consumers or followers will actively seek relative information).

ii) 'Persuasion - becoming convinced of the value of the innovation

iii) Decision - Committing to the adoption of the innovation 
iv) Implementation - putting the innovation to use

v) Confirmation - the ultimate acceptance (or rejection) of the innovation'.

According to Meadows, Rogers' proposed that would-be adopters go through each of the five stages 'before ultimately deciding whether or not to adopt [or reject] the innovation'. However, Stage 4 suggests that the innovation has already been adopted at this stage, as they are 'putting it to use'. It also suggests an entirely internal process, yet it relates to a common marketing communications model known as the AIDA model: Attention, Interest, Desire and Action [380]. Consumers gain knowledge through marketers who provide information about the innovation, or product, and create messages that stimulate interest, which can be likened to Rogers' Knowledge and Persuasion stages. The marketing messages should then generate desire for the innovation, which may be considered to be akin to Rogers' Decision to commit to the adoption stage. The marketing communication then prompts consumers to take action, i.e. purchase and use the product, which would align with Rogers' Implementation stage. The ultimate acceptance or rejection stage that Rogers proposes would then suggest some form of post-purchase evaluation, i.e. continuing to use or engage with the innovation, or not. Furthermore, Meadows confirms that Rogers believed that any individual's willingness to adopt an innovation, or trend, regardless of which category they belonged to for that particular innovation, requires a measure of 'awareness, interest, evaluation and trial'.

The evolution of the trend begins with the take up of the innovation, which according to Brannon and Divita [381] happens due to the 'trendiest consumers and entrepreneurial firms' who 'begin to participate' with the trend. For Raymond [382], the innovators are 'responsible for the development' or 'introduction of an idea'. This may suggest that the forecasters, designers and possibly even the buyers can be included in this sector, though Raymond goes on to suggest that the innovators 'may not be the ones who created the original idea, or kick-started the new trend, but they are the ones who can articulate it in a way which makes sense to other members of their tribe, or the group they come into contact with'. Furthermore, they are the 'first people a trend forecaster needs to track down within any community or sector if they are keen to identify a trend in its early stages and chart its progress through society as a whole'. Again, this suggests that trends begin on the street rather than being created and directed by the forecasters, or at least they lose ownership of the trend at this stage. For Keiser and Garner [383] the innovators have a more direct relationship with the fashion system and the introductory stage of promoting the new look. This would then suggest that regardless of how the forecasters anticipated the adoption of the trend, it is the innovators on the street that decide upon the context of the new trend and relates directly to how the trend will move forward. Bye [384], while discussing the role of the fashion designer and referring specifically to couturiers, notes that 'historically, couturiers presented new fashion [collections] twice per year, they had [once had] the power to dictate what was fashionable'. This may suggest that high fashion designers may be considered to be part of the innovator cohort, particularly as is the situation today, couturiers do not dictate fashion in the way they once did, but because their collections are watched and reported on around the world, 'they act as one set of visionaries for the fashion world'. Meadows [385] refers to the innovators as those who are the earliest to communicate 'a new style or look', and will do so to other consumers of fashion. Meadows also notes that the innovators themselves may not necessarily influence the taste of others, but will 
more simply create awareness and exposure. In this category, people are described as being 'more socially secure' than other group members and are more openly interested in fashion. They may also be described as being agents of change or as being innovative communicators of an innovation. Given that the individuals who are perceived as being innovators differs across product types, in reality, it is the levels of interest in the product and willingness to immerse in the trend that determines how one is categorised. Furthermore, Meadows identifies retailers, marketers and branding personnel as being the fashion leaders, as opposed to forecasters, and that the followers of fashion are the individuals who 'legitimise' the trend and therefore it is the followers that 'a trend truly relies' upon.

Brannon [386] acknowledges that forecasters will identify what they believe to be the next 'trendy' colours for a given season, though there is no indication of any assumptions of agreement on what those colours may be. Brannon states that 'the shock of the new attracts all but the most conservative consumers, but only the fashion forward buy these new colours as a total look' and that the remainder of the population, or target market will buy into 'small quantities as accessories, or in fabric prints or patterns'. How near, or far from the actuality this is may stimulate some debate. First of all, because we are part of a generation of people who have probably being exposed to all colours currently possible in our respective lifetimes, particularly due to the speed of change over more recent decades, what could possibly constitute a 'shock' colour? This surely must be put into some kind of context. For example, bright orange denim jeans may promote a shock reaction, but consumers may reject the jeans purely on the concept of finding them grotesque. Might it just be more coincidental that accessories with accents of orange may become popular in mainstream because consumers are more attracted to the other colours within the colour composition, or to be overall colour combination, and not even relate the small amounts of orange in the pattern to the aforementioned jeans?

The next group of innovative people are the early adopters. These are generally regarded to be those who have close connections with the innovators, and most likely to be friends or other individuals in the innovator's immediate circle of acquaintances, such as colleagues. Meadows [387] claims that the early adopters 'legitimise a style for fashion followers' and influence their social group members while at the same time, staying within 'the social norms' of their group. The early adopters are amenable to new products, new concepts, new ways of doing things and will quickly and easily follow their trusted innovator companions, they will also be connected to other groups of people, and according to Raymond, probably more so than the innovators themselves. Trend forecasters will also seek to identify and follow the early adopters [388]. Meadows also notes that early adopters may not necessarily engage with the innovation or trend fully, preferring modified or 'toned-down versions' of the look, and therefore not directly copying the innovators. Brannon \& Divita [389] describe this part of the process as the 'trendy' stage as trends become more visible 'and the most fashion-forward brands and retailers test the concept'. Once again, this suggests a trend that has developed at street level as opposed to being pushed through the fashion system from the forecasters and from the design process. Yet for Keiser and Garner [390] it is the product lifecycle that leads the trend through the adoption process. For Bye [391] price points are also an important factor to be considered when interpreting the fashion adoption cycle. There is no doubt that the early adopters are critical for trends to be adopted into mainstream fashion. What is likely to be happening in reality is that there are two trend scenarios 
operating simultaneously, the first being the 'more-organic' trends occurring on the street (in the natural environment), which are a source of inspiration for forecasters for future trends, and secondly, the manufactured trends once determined and promoted (forecast) by the forecasters, which have been adopted by the retail and manufacturing sectors and obtaining differing levels of acceptance by the general public as the trend moves through the adopter sets and product lifecycle.

By far the largest group of adopters of a trend are categorised as being the majority of a population. While it is recognised that individuals take up the innovation at different speeds, this group of people have been conveniently split into two segments of equal proportion, the early majority and the late majority. Raymond [392] describes the early majority as those who 'act as a bridge of reassurance between the early adopters and late majority', they are followers not leaders, yet they are important as they are the group of individuals who will keep the trend alive while the innovators and early adopters are moving on to the next trend. The early majority will be identified and tracked by the trend forecasters as they will be somewhat more selective and will determine the likely trends that the late majority will then adopt. Raymond also suggests that the early adopters may be used in panels or other types of test group for market research purposes. Furthermore, this is an important group for forecasters and marketers alike as it is at this stage when the trend can be more precisely determined and its longevity realised. One has to assume that this is part of tracking the existing trend while at the same time new trends are coming into being, but also the forecasters are creating new trends. Brannon \& Divita [393] relate this stage to mainstream fashion, which is further enforced as 'more conservative consumers join in, visibility continues to increase, and corporations and brands capitalise on the growing demand'. It is the more conservative late majority who demand even higher levels of assurance and also seek explanations of the trend in context to how they will benefit from it. This group of individuals are also amenable to modifications of the trend which Raymond describes as 'watered down' variations. This group are also easy to market to as they are the ultimately followers. They are however particular in their purchase choices and are known to actively seek style guidance, being influenced by media coverage and advertising, as they like to 'fit in'.

Meadows [394] provides a set of characteristics that define and separate the innovators from the early adopters and mainstream followers. The innovators are described as being: 'creative, participators, risktakers, they create their own identities, and have an unusual level of passion. They also have a strong interest in change and novelty'. Early adopters and mainstreamers on the other hand: 'want entertainment, [and want] to consume'. The early adopters and mainstreamers are 'spectators - just read about it, want comfort, to fit in, want safety, go where the herd goes, like stability, don't think too much'.

Furthermore, Meadows describes the late majority as being 'more sceptical' and that they like to take their time, needing to be convinced of the trend. Yet, like the early majority, they like to fit in and are very much followers, though there is a tendency towards tradition and often identified in lowersocioeconomic communities. It may be argued that early adopters are also, in a sense, leaders. For Raymond [395], the late majority 'are governed by social norms' as well as by the market and or economic influences. For this reason, people in this category can be quick to abandon a trend. They are also more likely to respond to bad press, either by the media or through word of mouth. This group of 
individuals are still important to observe as forecasters believe that they provide valuable insight into when and where a trend will begin to lose favour. The final category are the laggards. They are not particularly motivated by trends but do look for inspiration from those close to them. They favour easy wear and comfort. Some may also be sensitive to spending more than they can afford and are less likely to purchase on impulse.

As more of the general public buy into the trend, i.e. as the trend moves through the sets of people described above, so the product lifecycle curve, and fashion cycle, ebbs and flows, or rises and falls [396], which can also be used as a tool for tracking trends. This is more likely to be used by retailers though as it relates more directly to their sales volume. Meadows [397] notes that there is a 'need to identify trend direction [in order] to develop product accordingly'. Furthermore, Meadows [398] presents the fashion lifecycle phases as follows:

i) Innovation - fashion leaders will pay high prices for new looks

ii) Rise - more people start to adopt the key looks

iii) Acceleration - the look is copied by many retailers and adopted by fashion followers

iv) General acceptance - the look reaches its maximum sales potential and is to be seen everywhere

v) Decline - sales decline as new trends emerge; retailers reduce prices and start to replace the look with a newer trend

vi) Obsolescence - the look is no longer to be seen.

According to Kim, Fiore \& Kim [399], the fashion cycle can be used by forecasters and retailers to track trends by 'anticipating' their acceptance and duration. This would suggest that the tool does not in any way provide hard data in advance, merely a suggestion of possibilities.

\subsubsection{Planned obsolescence}

According to Sproles [400], at the time when a product has reached the saturation point in relation to the product lifecycle or the fashion cycle model, and is visibly observed within the marketplace. Consumers may tire of the product and will therefore be more receptive to something new. Where this happens naturally, the industry will instigate the change by replacing a withdrawn product with a new one, at least in theory. Sproles also recognised that manufacturing issues may enforce products to be made obsolete by the industry, though this is not very likely to happen with apparel as products are rarely so unfit for purpose or pose health and safety risks. Rather, Sproles suggests that consumers are more likely to 'render products obsolete through a change of taste, opinion or attitude' [401]. Also from a consumer perspective, Frings [402] proposes three principle reasons for fashion change: 1) changes to consumer lifestyles, 2) to relieve boredom and 3) other psychological need changes. Solomon and Rabolt [403] also refer to the changes in consumer taste as being a driver of natural product obsolescence, but they also recognise that the industry can influence the continued appeal of products, and likewise the discontinuation of designs, which of course is achieved through the promotion and marketing of trends. It is this practice that is known as planned obsolescence. For Yurchisin and Kim 
[404], planned obsolescence influences consumers in a negative way instigating feelings of dissatisfaction, and deliberately promotes and, or, reinforces the sense of wanting something new.

As a business strategy, planned obsolescence is not confined to the fashion industry, many consumer product industries will use design, including colour, to make current products undesirable in order to make way for new versions of the product to enter the marketplace to create new sales. However, because the fashion industry favours 'extremely fast cycles of fashion and consumers' unsustainable desires', Niinimäki and Hassi [405] prescribe the clothing industry to be one of the best examples of planned obsolescence in the wider industry system. Bulow [406] aptly describes planned obsolescence as being 'the production of goods with uneconomically short useful lives so that customers will have to make repeat purchases'.

Colour forecasters and trend forecasters often provide forecasts for a number of industry sectors. Therefore, through trends, obsolescence is 'built into' the design of the product [407]. Planned obsolescence is essentially a shortening of the lifetime of a product within the product lifecycle and fashion cycle [408]. For fashion product, making an existing product appear to be outmoded lessens its fashion-ability and therefore promotes obsolescence. Of course, there is a risk in that the new product design may not be favoured at all by innovators to promote the new trend, or is not amenable to the mainstream and therefore the product will not move through the product lifecycle beyond the introductory stage. Originally planned obsolescence in the context of fashion was a seasonal practice [409]. Today, new product, and therefore new trends, may be pushed through the product lifecycle within a number of weeks rather than months to drive sales. Kim, Fiore \& Kim [410] notes that it is the promoters that push a new trend through the product lifecycle through the marketing of the trend creating awareness and visibility. Fiore [411] describes the promoters as professionals, whose role is to emphasise and enhance the aesthetic of a product using verbal communication, or to design appropriate environments to support the product or brand. For Niinimäki [412] this relates to 'the psychological obsolescence that consumers themselves [will then] feel about the product'.

While literature suggests that trends decline due to 'market saturation, social saturation (overuse), boredom, the desire for novelty, loss of prestige or exclusivity' [413], for Sproles [414], planned obsolescence is led by industry, and, what he terms as 'social obsolescence', is 'forced by social change'. Therefore, there is more than just one force that can be involved, yet further discussions regarding this as a process is lacking. Waldman [415], on the other hand, offers further insight through the following two abridged examples after having declared that 'in many settings the incentive for a durable goods monopolist to introduce new products that make old units obsolete will be above that which is socially optimal':

1) 'Devaluing previous product': through the example of a textbook publisher we are invited to consider the publisher of a popular textbook who periodically introduces new editions of his book - From our standpoint, there are two important decisions faced by this firm. First, [they] must decide how often to introduce new editions, where the introduction of a new edition results in a discrete drop in the value of old copies. Second, in revising the book he must decide how similar to make the new edition to the current edition. What is important here is that a 
more dramatic change will make it more difficult for [an individual] to continue to use an old copy, and hence, will have a bigger negative effect on the value of old copies. Another way of putting the above is that, every period in which the firm sells copies of his book he must decide how compatible to make new copies with old copies, and his choice concerning compatibility affects the value of copies he previously sold. This suggests that, both from his own private standpoint and from a social welfare standpoint, this firm's incentive to make new copies incompatible with old copies will be too high. In other words, the firm will have an incentive to bring out new editions too soon, and when he brings out a new edition, he will have an incentive to write it in such a way as to kill off the market for old copies'.

It is easy to relate this to how fashion trends are manipulated by the industry in order to essentially sell more of the same product but in a manner that renders previous product unfashionable.

2) 'Style changes': changing the appearance of the product - 'In some circumstances, a firm will have an overly high incentive to introduce style changes into its new units of output'. This applies very much to the fashion industry. 'The logic follows, suppose that consumers place a lower value on old units when old and new units differ in terms of their appearance. A firm faced with customers of this sort will face [a] time-inconsistency problem because the firm does not internalise how its behaviour affects the value placed on old units, its incentive will be too high to behave in a manner that lowers this value. What this means in the current setting is that, both from its own private standpoint and from a social welfare standpoint, the firm's incentive to introduce style changes will be too high. One might object that I am missing an important part of the story. In a setting where consumers place a lower value on old units when old and new units differ in appearance, it will also typically be true that consumers place a higher value on new units that have a distinctive style. Incorporating this aspect of the story into the argument, however, has no effect on the final conclusion. The reason is that the positive effect that a style change has on the valuation of new units will be internalised by the monopolist, while the negative effect on the value of old units will not be internalised. Hence, even if a style change has a positive effect on the value placed on new units, the firm's incentive to introduce style changes will still be too high'.

What is of particular interest here is that Waldman takes into consideration the consumer, or social welfare of the product user, in a way that forecasters do not, yet they appear to try to justify that change is required because individuals demand it and that the colour evolution tool, the fashion pendulum, can be used by forecasters to help manage the change. Where in reality industry has a far greater need for change to drive sales than the general public needs. This is evident in the fact that there are many stages of progression, and consumer types (innovators, early adopters, etc.), through the product lifecycle and fashion cycle to bring a trend to full saturation and into decline. The following Section focuses on the adoption theories used by forecasters. 


\subsection{Adoption theories}

Marketeers recognise that the consumer is at the heart of all adoption theories. The adoption of trends is akin to the same stages of marketing communications that drive sales. At the stage of a new product launch, or the onset on a newly marketed trend, consumers are said to be taken from a stage of ignorance to a stage of awareness. The stage of ignorance is when the forecasters are still developing the next trend concept to be promoted, and awareness can begin when the trend is made visible to the general public, though mostly to the trend-setters or innovators who will ultimately drive the trend through the aforementioned adopter categories (Section 7.2). The next stage of the adoption process is the creation of knowledge to support the trend. This is largely the role of the fashion media which is further reinforced by fashion innovators. Social media and bloggers have increased the speed of communications and perhaps there is already a blur between innovators and early adopters, or at least a very short time span between these two sets of adopters engaging with the trend. In theory, this is followed by the stage of liking. In reality, the speed between moving from the stage of knowledge to the stage of liking will be minimal. Liking generally involves cognitive reasoning and the desire to engage, though may be on a subconscious level. Interestingly, the next stage is preference. In marketing terms this relates very much to product placement; being in the right place at the right time. Trends, or the formulation of trends, are notoriously detached from consumer preferences and therefore the power of the individuals who operate within the innovator stage, which was identified within the diffusion theory is particularly critical in order to take consumers to the next stages, which are conviction and adoption, or purchase [416]. A further set of fashion trend specific theories of adoption are commonly accepted under the guise of the trickle theories which are discussed in the following Subsection.

\subsubsection{The trickle theories of adoption}

The adoption theories are the predominate causes of a trend movement. Where marketing theories focus on the mind-set on the individual, the trickle theories relate more to the fashion leadership theories that put sets of individuals at the core of the concept. The trickle theories comprise of trickledown, a downward flow from the innovators to other adopters, trickle up, which has an upward flow from a different set of innovators to other adopters, and trickle across, which is so called due to its sideways, or horizontal movement [417] of the trend adoption. More recently the theories have come under scrutiny as far more significant changes in social environments and in general market conditions, plus consumer preferences have been recognised; and the way in which consumers find information and engage with trends has also altered significantly since the development of these particular theories [418]. However, it is still considered to be important for forecasters to understand the underlying principles of these theories, which are still much referred to, in order to fully appreciate all of the potential drivers of a trend movement, and particularly how trends have moved in the past from which to model the direction of trend movement and change.

Trickle-down theory is reputed to be the oldest of the three trend-adoption theories. It begins with the social elite as the fashion innovators and promoters of a new style or colour trend, which is subsequently copied by the lower social classes, thus trends spread from the top down [419]. This method of adoption can be seen throughout the centuries prior to the twentieth century, which may 
have been as a direct result of garments been handed down to maids and to other serving staff by the wealthy classes. In more recent times, with the establishment of couture houses and the more familiar fashion system of today, influential fashion designers were also considered to be the catalysts for new trends that become watered down for the purpose of mass production [420]. This latter aspect is considered to still validate the theory today, but the social-class-division thinking is less favoured and often disputed by historians and fashion analysts. However, the principle can still be justified as previously stated throughout the diffusion theory discussion (Section 7.2.1), as trends move to the many from the few, regardless of status. It would appear that today the instigators of trends encapsulated by the trickle-down concept are individuals that others seek information from and look up to for inspiration, regardless of their background, place in society and earning capacity. Kim, Fiore and Kim [421] pinpoint the 1960s as being the pivotal point in time when a change began to happen, and the single trends that were imposed on others from the top of the social pyramid to the less wealthy was not the only model of trend adoption. Though it is equally recognised that such a simplistic movement of trends makes it far easier for anyone to observe the movements and to therefore predict future trajectories. However, as mass-production further developed and fashionable products were more readily available and more affordable to many, different subcultures also developed. As each adopted their own sense of style this led to other modes of fashion diffusion and adoption being recognised. According to McCracken [422] trickle-down theory may be likened to the concept of chase and flight, where lower classes seek to imitate (chase) and the elite seek to adopt new styles (flight) to differentiate. Essentially, the trend moves down from an 'upper strata of society to the general population becoming a universal fashion movement' [423].

As the name suggests, trickle-up theory is completely the opposite to trickle-down theory as the trend begins its life on the street and moves upwards to the couture designers [424], who then re-interpret the style, which is further re-interpreted by designers in the mass production sector for the mainstream market. Subcultures are therefore an important catalyst for style, which both designers and forecasters identify through their observatory research. Subcultures are also catalysts for trend movements across different subcultures, which is aptly known as the trickle-across theory, which was recognised by King in the early 1960s [425]. This works where there are similar tastes between sets of individuals, or where the same message is to be expressed through clothing. Essentially, trickle-up and trickle-across theories begin with small sets of individuals on the street adopting and adapting their own style, often through their subcultural experiences as they experiment with a look to create their own identity. This then acts as a point of inspiration for fashion designers and trend forecasters. These sets of individuals are of particular interest to trend forecasters who work more with macro trends, as their behaviours inform trends that transcend beyond just fashion. Regardless of the labelling of the theory, forecasters must determine a way of working with trends; to identify them and their adopters, to track their movement and to accurately evaluate the likely trajectory of the trend; as well as to understand, anticipate and monitor other influences that may trigger new trends. Forecasters do not invent trends, rather, they facilitate the marketing of trends that are instigated by creative and innovative individuals who may be designers, iconic idols or, and maybe more likely today, members of the general public who strive for a unique identity within a manufactured existence and environment. For the industry, trends that trickle down have gained exposure and favour with innovators and early adopters, which helps to move the 
trend into mainstream more quickly, and thus create early sales followed by more substantial sales volume when the trend is adopted by the mainstreamers. On the other hand, trickle-up trends must be correctly interpreted for the mass market and may require additional marketing efforts to convince early adopters to buy into the trend, as these trends are likely to be more relevant to subcultures. Consequently, the more the industry can take control of the trend from the onset, the better the chances are of the trend establishing in the mainstream.

\subsection{Colour and trend movement}

it is unclear whether forecasters engage with the process of actually recording and tracking change, as opposed to simply observing and memorising observations. Kim, Fiore \& Kim [426] refer to individuals as analysts rather than forecasters. They propose that using diffusion curves, as discussed in Section 7.2, 'the analyst' can 'estimate' the extent of a trend. Identifying the point of the trend moving from the innovators to the early adopters in order to impact on mainstream acceptance appears to be critical for analysing the rate of acceptance.

Vejlgaard [427] has also discussed the speed of trends; for him the process is slightly more complex stating the trends dependence on 'any number of factors .... [that] should be noted at all times'. Vejlgaard relates the speed of the trend directly to its visibility and the ease of being able to observe the trend. This would explain why there is substantial growth in the diffusion curve when the trend is experienced in mainstream. He also notes that it is at this point that the trend is easy to adapt. This would suggest that it is at the point of becoming a mainstream trend when designers and product developers engage an interest, rather than at the point of receiving the insight ahead of time through the forecasters. Hence, it would appear that designers wait for the trend to develop and take this as a cue to take the trend into mainstream production, which suggests the need for the verification of the forecasts before investing in the trend. Trends that move more slowly are supposedly those that have an emotional dimension, which of course includes colour given that we have preferences for colours that have some kind of psychological meaning for us. Trends that are of a subjective nature, such as ecofashion, also move much more slowly. This may be because they rely on individuals genuinely becoming interested in the movement without the industry's intervention in promoting the trend, with the intention of it being a short-term trend. Furthermore, for Vejlgaard the observation of trends 'must be accompanied by a process of synthesising [in order] to map [it] accurately'. He has devised a trend model that is referred to as the diamond-shaped trend model, which actually resembles the appearance of two isosceles triangles joined at the base of the first with the second being a mirror image, rather than looking like a cut diamond. At the top are the trend creators, those deemed to be 'extremely innovative and inventive', suggesting that there are relatively few in the world. Then there are the trend setters beneath the creators, these individuals instigate the movement of the trend. Then there are the trend followers and the early mainstream, and the larger mainstream segment of people are at the widest point of the model and continue into the narrowing inverted triangle area. There we have the 
late mainstreamers and then the conservatives. At the bottom point there are, what he terms, the antiinnovators. This is really just another way of expressing the diffusion of a trend through a set of different characters that represent the population in more general terms. Regardless of the model used, observation is still probably the most critical tool for identifying trends and their movement.

As previously mentioned, at the stage when designers appear to engage with the trend is where the forecast information becomes more meaningful to designers. As McAssey and Buckley [428] note, the purpose of the forecasts for designers is to provide them with a tool to identify colour change and the direction of this change. Interestingly, if colour forecasts could be relied upon to be $100 \%$ accurate then the trend analysis stage could be applied directly through the forecasts without the need for designers to also undertake some of the role of the forecaster to observe the movement, direction and speed of trends. Perhaps for the cynical, this idealisation is essentially what they believe should be the purpose of forecasts, and because designers, buyers, etc., still have to watch trends for themselves, the forecasts are considered to be not-fit for purpose. It should still be remembered however, that designers will use the forecasts as guidance for 'seasonal newness' but the colour palates that the designers will create are more tailored to the needs of their company's branding image and their target market's desires. Keiser and Garner [429] note that the new colour story suggested by the colour forecasters may take a while to appear within the marketplace, and further suggest that this links to the concept of colour cycles having an approximate three-year span, as different brands engage with the new palette at different times in the product lifecycle stages. For example, more fashion forward brands will use the colours much earlier on in the lifecycle than mainstream retailers. The cause of the trend is stated to be important by Wolbers [430], though this is not elaborated on, and for Stone [431], a trend in itself 'refers to a general direction or movement'. Perhaps what Wolbers and Stone mean is that a trend cannot be stagnant, it has to come into being, to ebb and flow and at some point to disappear, and for this to take place the trend must be influenced by one or more factors. Those factors may to some degree be reasoned through the theories of adoption discussed earlier, and possibly more interesting for some, is how trends can resurface at different periods in time, otherwise known as cycles.

The rate of adoption of a trend may also be related to the theory of product adoption, as discussed by Rath, Petrizzi and Gill [432], who propose a five-stage process, that is not entirely dissimilar to the AIDA model discussed in Section 7.2.1 in relation to the diffusion theory. The product adoption theory is particularly of interest to marketeers, as Rath, Petrizzi and Gill note, some consumers will exit the cycle of adoption at any one time and from any of the proposed stages. Because of this, it is somewhat easy to understand why trends can move at different paces before disappearing.

The five stages are as follows:

i) 'Awareness - you hear about some new product but don't really have much information about it'

This may be something word-of-mouth or having seen some kind of visual reference to the product or trend which would likely have been instigated by the innovators.

ii) 'Interest - you check various sources to learn more' 
This is probably more likely if you are one of the early adopters, for the majority of the population interest will not be sparked until there has been more media coverage or if it has struck a more emotional chord for some reason.

iii) 'Evaluation - you consider its product attributes and think about whether it is something you really want or need'

This would suggest that individuals are considered to be more analytical in their thought processes rather than simply being followers.

iv) 'Trial-you give it a chance and try it for the first time'

It is at this point for colour trends in particular where the interest may be short-lived and the product ends up in the disposal / recycle phase that is currently missing in product lifecycle models.

v) 'Adoption - you start using it consistently'

This is the point where trend cycles are created.

The product lifecycle and the adoption of the trend within the lifecycle is used as a tool to analyse the movement of a trend through consumer choice. Fashion trends can be determined as being a 'temporary cyclical phenomena' [433], as individuals engage with the trend for a period of time within particular situations. A taste for a trend can return, though not necessarily for the same set of individuals, or for the same type of product and anticipating the return of a hue and the quality of hue is of particular concern for colour forecasters.

\subsubsection{Colour cycles}

According to Brannon [434], while trends come into fashion, leave and return at some point, such cycles have no fixed, regular recurrence. Colour cycles may be identified through the movement of particular hues, or changes in intensity or value and through the repetition of their reappearance. Classic, or timeless colours will remain favourable for much longer than fashion or fad colours, which also demand a higher quality of product in order to be sufficiently durable for longer lasting use [435]. Throughout the twentieth century, up until around the late 1980s, colour cycles appeared to have a general time span of an approximate five-year duration [436]. Though this had already begun to decrease before the millennium, which may have coincided with the increasing popularity of the fast fashion production model used by the industry. Leonhard Oberascher [437] observed colour cycles in architecture as having a fixed order of colour preference which moves from multi-coloured highly-chromatic hues to more subdued hues and earth tones, to achromatics and then beginning the cycle again with multi-coloured high chromatics. A similar pattern was observed on the covers of a German interiors magazine covering a twenty-year period of the latter decades of the twentieth century, the cycle being high-chromatic hues, multi-colouredness, dark colours, earth tones, light colours, white, achromatics, a return to high chroma, purples and more high chroma colours to begin the cycle again. At times particular colours are on-trend, for example Kenneth Charbonneau [438] spoke of the avocado years (of the 1970s), which were supported by harvest gold and copper tones. The colours moved into earth tones with a green bias and then moved to chocolate brown. The oranges of the avocado years moved to terracotta and further orangey earth tones. Colours generally then moved to pastels and creams, and greys replaced browns before moving into mauve and rose. Brannon [439] recognised a similar colour movement in fashion 
forecasts from the earlier part of the twentieth century having moved from multi-coloured high chroma, to earth tones, to achromatics, to purples, and back to multi-coloured high chromatic hues. The subtle changes based on hue, intensity and colour temperature moved colour through the cycle and an approximate seven-year lapse was identified between cooler hues and warmer hues. By the millennium it was recognised that colour cycles were more typically lasting around three years as they gained saturation in the marketplace more quickly before disappearing [440]. For Rousso and Ostroff [441], colour cycles are 'shifts in colour preferences and colour repetition', suggesting that consumers may be more in control of colour change than forecasters or designers. They reiterate the cyclic pattern of colour in fashion as being high chroma, multi-colouredness, earth tones, acromatics, the purple phase and back to high chroma; and that colour acceptance moves from warm colours to cool colours. However, it would appear that very few studies of colour cycles exist and that perhaps this prescription of colour change has been largely accepted without further verification. This would suggest that a more thorough investigation into the cycles of colour is probably overdue. In addition, colour revivals may be seen to correspond to fashion history and can be determined to be underpinned by economic climates, cultural and societal influences. For example, during economic instability people have a tendency to seek security, at such times earthy tones have tended to become more popular [442]; and 'in times of uncertainty, consumers are most comfortable with colours that are a reminder of familiar things' [443], yet there is currently 'no clear method in place to fully understand the colour evolution process' [444].

For the forecasters, and for designers, buyers, and other industry personnel, understanding colour cycles is very important from a trend perspective through the shifts and repetitious patterns of colour preferences and the popularity of colours. For the colour forecaster, the rhythms of movement and the timing of change is of particular value, as are comparisons of palettes on a seasonal basis to inform future palettes. As consumer colour preference change is recognised to link directly to value, chroma, and colour temperature differences forecasters need to work within the parameters of these relationships [445]. However, with such speed of change demanded by industry for continued sales, perhaps a new service is now required that focuses solely on colour cycle research to better inform the forecasting sector of colour preference and acceptance change.

\subsubsection{Classics and fads}

The length of time a colour remains popular by the majority of the market population is a critical aspect of a colour cycle and of a colour forecast. The terms classic and fad are used in reference to the approximate length of time a colour will be acceptable for at the two extremities. A classic is generally accepted for a long or indefinite period of time, black is a typical example. Such colours have long-term preference, though not necessarily for all product types at the same time. Staple colours are also preferred in the long term. These are used as wardrobe basics, including black, white and greys, navy, browns, beige and cream. They are still largely determined by the forecasters for particular future seasons along with sets of fashion colours.

Fads have the shortest period of acceptance. These can be particularly risky for retailers as the colour can lose favour incredibly quickly. These are essentially fashion colours and a main aspect of a colour forecast. Colours that appear very quickly rather than evolving into being have more potential to be fads 
as they may appear to be out-of-their-time; it may be considered very bold to wear it and may not be fully supported by the type of personality to continually carry it off. Alternatively, the fad colour may have been simply considered to be a whim by the consumer or only appropriate for a particular event, consequently the acceptance of, or engagement with the colour last momentarily. Some colours may not even become a fad, as still enough people must adopt it, albeit fleetingly, for it to be recognised as a fad, otherwise it will simply be considered to be a flop and a total disaster for industry. The challenge therefore for forecasters, designers, buyers and other fashion and textile industry personnel is to determine the strength of colour acceptance and to understand colour preferences and to accurately sense the position of the colour within the trend zeitgeist to substantiate any investment, both for industry and for consumers [446].

Briefly, a further consideration is the concept of cycles within cycles, this is when newness is offered through the manipulation of an aspect of design, where the outcome is still recognised as being a part of the trend, yet changed enough to be different and still acceptable [447]. Cycle within cycles are mentioned as part of the overall discussion of cycles in literature but does not appear to be really considered with specific reference to colour cycles. This is probably due to a lack of any substantial research to support the concept for colour forecasting.

\section{Further analytical trend analysis techniques}

Martin Raymond is the co-founder of The Future Laboratory, a UK-based consumer insight, trend forecasting and brand strategy agency [448]. According to Raymond all trends can be identified and tracked, and can therefore be predicted. It is simply a process of 'anticipating' cultural shifts. There is a need to understand what the trend is to know where to look and what to look for, but perhaps more critically for Raymond is knowing how to 'read or braille' trends. The tools proposed for such brailling are more sophisticated than the more-objective tools that were discussed throughout Sections 5 and 7 , which include 'neuro-psychology, memetics, behavioural economics, complexity theory, scenario planning, network science and ethnography', plus 'strategic intuition' [449] (perhaps more detailed than the discussion of intuition in Section 5.2.3). Indeed, Duggan [450] speaks of strategic insight as being very different from 'ordinary' intuition, in that it is thinking not feeling. Where ordinary intuition involves gut feeling or vague hunches, strategic intuition are the flashes of clear insight at the core of what may be happening; it is strategic, deliberate not random - involving conscious deliberation not flashes of insight from the subconscious mind. However, just because a colour forecaster, or fashion trend forecaster has not expressed or declared their intuition type it cannot simply be assumed that colour, fashion and textile trend forecasters all operate on a superficial level compared to the trend forecaster type that Raymond speaks of, those who attempt to forecast the often slower-paced macro and mega trends that are further discussed in the following Subsection. Also the suggestion of neuropsychology being a forecaster's tool may be considered to be somewhat unrealistic as a scientific study of the brain and its functioning for the purpose of understanding human behaviour [451] can be considered to be beyond the realms of what a forecaster does, can do, or should do. What Raymond may be suggesting is perhaps the use of the theories or some of the understanding that underpins those theories to better understand behaviours. Similarly, memetics, principally the study of culture, though grounded in Darwin's theory of evolution [452] may also be considered to be unrealistic in relation to 
what forecasting entails, or a technique beyond the colour forecasters' tool kit, and the area of memetics is still very much open to debate. Remember that the trend forecasting sector has grown substantially since the 1960s and 1970s and therefore forecasting agencies need to differentiate themselves from their competitors just as companies in all industry sectors must. In order to differentiate and to appear to be better than competitors, the methodology claims of forecasters may be used to substantiate their 'better-ness' to secure a broader spectrum of clients. This, as a marketing strategy has clearly over-complicated the trend forecasting process and at the same time may also have made fashion and textiles companies feel that they need as much 'expert' advice and direction as they can muster. Let us also not forget that the bigger the trend forecasting company, in terms of its clientbase, the more generalised the forecasts are to appeal to a more global clientele. In reality, as indicated in the final Section of this Textile Progress, tailored forecasts for individual companies may be more valuable to designers and retailers in terms of brand recognition and brand loyalty, as they could better meet the consumer preferences of their target market, and in order to 'de-homogenise' fashion on the high street, which would generally inspire a more creative and diverse offering. The more important technique to take away from Raymond's list is perhaps ethnography, which is covered in more detail in Section 8.5.

\subsection{Social and economic trends, and mega trends}

As previously stated, trends are not predicted, but identified [453]. It is also important to understand that colour forecasting, while a niche sector within the broader fashion forecasting sector, is also part of a larger body of trends that happen independently of fashion. It may be useful to think about colour forecasting at the centre of an onion, where other trends form layers from the core that become larger through diversification, and in essence, different types of forecaster exist and focus primarily within a layer, yet they inform other layers (see Figure 16). The extent of how much one layer informs another layer is at the discretion of the forecaster or of the forecasting agency, relevant to their company mission statement and strategic vision for the company.

Where pan-cultural or universal trends happen: Mega Trends

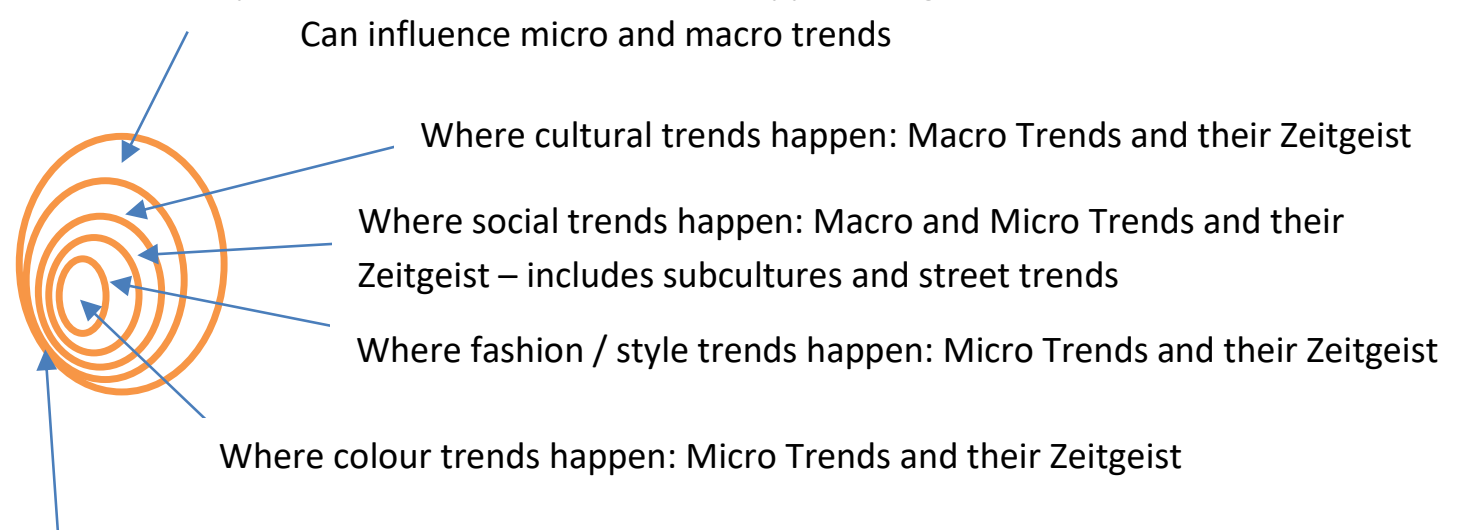


Figure 16. The colour forecasting trend onion (Source: Author's own interpretation)

Colour and fashion forecasters need to work with the larger-scale social trends, to understand the cultural trends and to be aware of mega trends and the economic landscape for the intended season. Other macro-trend information, such as new advances in the dyeing and textiles industries and technological influences which may include communications as the micro-, macro- and mega-trends all inform lifestyle changes and consumer preferences that then impact on personal choices such as colour and style, all of which become identifiable in the zeitgeist. As Mbonu [454] notes, 'trends emerge from societal issues that penetrate all walks of life', and that macro trends are a consequence of changes in society, an aging population is given by way of example. For the colour forecaster it is therefore imperative to know colour preferences between groups of people. This is elaborated on in Section 9.

Mega trends have global impact and therefore influence societies and cultures, economies and business practices and impact on other macro-environmental factors, the main ones being political, legal, ecological, technological, educational and demographical implications [455]. The term megatrend was probably first used by the consultant and futurist John Naisbitt in the early 1980s [456]. His book, Megatrends: Ten new directions transforming our lives, although he was addressing global trends that he had identified, it was originally written for an American audience and yet it captivated readers around the world [457]. Mega trends are trends that happen within the macro-environment and are therefore not under the control of the forecaster. Population shifts are of particular importance for fashion forecasters as part of the larger social shifts that impact on demographics, geographic locations, ethnicity and diversity, which further impact cultural shifts. Trend-forecasting agencies, such as The Future Laboratory, aim to decipher these trends for the colour forecasters and fashion trend forecasters, which serves to further layer the forecasting sector. Other considerations that trend forecasters pay attention to are ethical and social responsibility interests as communities and societies change their perceptions of the global challenges that fashion has imposed [458]. The diversity of political stances imposed not only by Governments but also by celebrities and bloggers adds substantial weight to forecasters having a vested interest in the macro- and mega-trend layers of the simplistic colour forecaster's onion in Figure 16. Forecasters whose primary concern is the analysis of the macroenvironment: social, economic, geographic, technological and demographical shifts, is termed the population trend forecaster according to Granger [459]. It is the colour forecasters and fashion trend 
forecasters who interpret this broader trend information into the product context for designers, product developers, buyers, merchandisers and retailers.

\subsection{Fashion scanning and fashion analysis}

Colour and fashion forecasters work mostly with micro trends where popular culture plays a significant factor in the trend analysis, while still paying attention to the larger-scale social and economic trends. Consumer lifestyle is an important consideration and relates to the discussions in Section 9. Angus \& Jhally [460] discuss culture in terms of class, high-class culture and the mass-form popular culture. While apparently not really of consideration amongst forecasters, it could be construed that there are two critical areas of inspiration for trends that could be appropriate for en masse trends and for high fashion (haute couture), or luxury fashion. This could be considered to be an unidentified aspect of the trickledown theory discussed in Section 7.3.1. It could also be considered as a niche way of trend forecasting that has not as yet being considered. Also the consideration of cultural consumption, or cultural context having an impact on preferences being regionalised, perhaps linked to religions, as well as being generational and having ethnic implications, as identified by Gans [461] will be further contextualised in Sections 9 and 10. In this Section we pay lip-service to these wider cultural considerations in order to focus more carefully on the concepts of fashion scanning and fashion analysis. It should also be made clear that today fashion scanning in the context of trend forecasting is not directly related to body scanning, or 3D body scanning that serves to support the fashion industry, as interesting as this growing body of research is, as yet such technology does not need to concern forecasters of colour and style needs; though may be someday soon a fashion forecasting agency may claim to include such technology in its methodology as a diversification marketing strategy!

For Brannon and Divita [462] a fashion scan is simply absorbing and engaging with newsworthy articles that report on the activities that are current in the fashion world, or fashion-business world. Such resources can be used to help to identify emerging lifestyle preferences and changes, however, popular culture also informs this and it is therefore necessary to be connected with music, theatre, film, TV and sports, as well as knowing up-to-date information in related technologies. Gathering and analysing such data should be an on-going iterative process. Scanning is used in the same context as Raymond's brailling, discussed at the onset of Section 8. It is a simplistic term to cover all of the research methods used for the data collection processes used for trend spotting (see Section 4.5.1), including, for example, observations (see Section 5.2.1). The analysis not only includes bringing trend clues together but also must be considered in relation to consumer readiness for a new trend [463]. Fashion analysis brings together all of the insights within and beyond the fashion industry sector and consumer behaviour to be translated into product, from the fibres, yarns, fabrics through design, including the use of colour, and the manufacturing and the retailing of the products thus informing beyond the trend forecasting sector, yet providing essential knowledge for forecasters. Tracking the origins of trends and carefully monitoring the speed and direction of change is paramount, particularly as trends can appear almost instantly and disappear equally as quickly [464]. For some, fashion scanning may simply be keeping abreast of what is happening in the world of fashion, something that fashion professionals should be doing anyway. 
What is not included in the literature that gives specific mention to fashion scanning and fashion analysis that is of particular benefit to the colour and fashion forecaster is fashion-design analysis and scanning or more particular to the colour forecaster, colour-design analysis and scanning. Much of trend forecasting hangs in the balance of evolution, the progressive direction of change rather than sudden, random changes. While it is important to have knowledge of the wider implications that can influence the make or break of a design, or acceptance of a colour, truly tracking and monitoring the evolution of colour and fashion-design change, and how this relates to the cyclic nature of design and the zeitgeist, is still very much under-researched. This raises the question the validity of all of the extended forecasting methods and paraphernalia now packaged into the trend-forecasting methodology and perhaps its overall philosophy, which may be in danger of eroding the power of colour and fashion trend forecasting.

\subsection{Environmental scanning}

Forecast companies such as The Future Laboratory have heavily promoted themselves as being, not only masters of trend forecasting, but also of consumer insight intelligence and 'strategic foresight' [465]. It is the impact of trend change and new emerging trends within the macro-environment and in particular within the cultural context that directly influence micro-trends, in for example fashion, but also through other factors such as technology. For some, environmental scanning and analysis is an extension of fashion scanning and analysis that involves observations in the major fashion cities around the world, as well as using media and the internet as a resource for trend spotting. According to Brannon and Divita [466], scanning the environment moves beyond scanning specifically within the fashion business context to include all manner of business practice, searching for new, novel and unusual, in terms of both practice and products, and also observing what people are doing, for example, where they are visiting or taking vacation that is very different from the conventional norm. It also includes observing new shopping habits and destinations, the way people work, how they engage within communities, neighbourhoods and cultures. Really, environmental scanning is a specific label for the forecasters' soft techniques discussed throughout the Subsections of 5.2 but makes these processes sound more official and important. For some, more precise terminology may confirm a need for forecasting, for the cynical it may reinforce the forecasters' need to remain relevant for the industry it serves. One may wonder when a term such as a super-trend forecasting agency may appear on the landscape of this ever-growing competitive sector, as bigger and more diverse in the methodological approach taken by forecasters has been 'the trend' in recent decades. As with the fashion pendulum discussed in Section 7.1.1, the trend for big, global and universal will at some point reach its extremity and the tide must turn towards smaller, more tailored and local forecasts. We return to this concept in Section 10.

\subsection{Market research methods}

The market research methods used by forecasters are akin to those used more generally by marketeers. The methods provide the forecaster with datasets to analyse in order to identify the emergence of trends and to track the speed and direction of their change. Past sales data, privy only to individual manufacturing and retail companies can be used quantitatively, though the majority of data collected by forecasters is qualitative. Beyond observations, interviews and focus groups are declared to be 
commonly used [467]. However, for Raymond [468] focus groups really only serve to confirm what is already known rather than as a method to reveal something new. It is considered by others to be a useful exercise to undertake as the confirmations serve to reinforce what is known of the trend and therefore reduces the risk factor. One such method of confirming attitudes and beliefs is known as a projective technique where prompts are used, for example incomplete sentences, word association tests or the use of images to prompt participants to tell stories [469]. Projective interviewing techniques employ indirect questions, such as asking a person to comment on what others may do or think rather than asking them to disclose what they do or think. Such techniques can facilitate lateral thinking [470]. Data mining has also become an important tool for some forecasters. This is a technique used to identify patterns in consumer behaviour from databases using neural network and parallel processing technologies [471], which may incidentally be what Raymond was proposing to be using, as discussed at the onset of Section 8, and may provide an interesting new method for colour forecasting in the near future as the collection and use of big data is also becoming a marketing strategy trend. As yet, it would still probably be limited to capturing what is already visible to be observed, as opposed to having the ability to understand colour preferences beyond current availability in the marketplace.

For the colour forecaster and fashion forecaster, demographics and lifestyle trend data for target consumers can be beneficial, which complements observation data. The use of market research data has grown since the 1960s and 1970s when fashion forecasters are now professed to have been akin to today's trend spotters. The approach to fashion forecasting has become more systematic and a wider set of information is now used and said to aid the relevance of a trend to particular target markets. For Levy and Weitz [472] lifestyle data is more beneficial for forecasters than demographics data. However, as much as such techniques are recognised to be used by forecasters, how they are actually employed and to what extent are never really divulged. The techniques are not specific to forecasting, rather they are borrowed from market research practice and discussed in a very light-touch and superficial way. Considering the speed at which the fashion industry would like trends to change, and are manufactured to change for the continuation of sales, it would not give much time for forecasters to undertake a much more detailed data collection and analysis process beyond the time it would have taken the trend spotter-forecaster of the 1960s. Even with the availability of the internet as a data resource and sophisticated data analysis packages, and taking away the additional forecasting layers that have materialised over time through companies such as The Future Laboratory, in reality, colour forecasting and fashion forecasting today is probably not greatly different to its predecessor of the mid-twentieth century; and that the growth of the layers of the colour forecasting 'trend onion' (Figure 16) have overcomplicated a more simplistic process. Furthermore, the recognition of the importance of communities of people, such as subcultures and the need for expressing individuality through personal preferences offers a far more appropriate type of forecasting for fashion-related product than the more convoluted mixed bag of approaches that generally appear to be suggested by authors writing about the trend forecasting sector.

\subsection{Ethnography}

Unlike fashion scanning, which is essentially a secondary research method, ethnography is a primary research method where attempts are made to gather first-hand data regarding a topic within the 
boundaries of a specific setting. Both Raymond [473] and Rath, Petrizzi and Gill [474] confirm the use of ethnography in the broader trend forecasting process and in the fashion forecasting process respectively, as an observational research approach. Rath et al, describe ethnographic research as being a method for studying the natural behaviour of consumers to reveal facts that could not otherwise be observed. Bryman [475] defines ethnography as being a process were the researcher becomes immersed in a social setting to observe behaviour and that often the researcher will also use other data collection and analysis methods. Bryman sets criteria [476] particular to his book as it is recognised that definitions differ amongst authors. For Bryman, the ethnographic-researcher does all of the following:

i) 'is immersed in a social setting for an extended period of time

ii) makes regular observations of the behaviour of members of that setting

iii) listens to and engages in conversations

iv) interviews informants on issues that are not directly amenable to observation or that the ethnographer is unclear about (or for other possible reasons)

v) collects documents about the group

vi) develops an understanding of the culture of the group and people's behaviour within the context of that culture

vii) and writes up a detailed account of that setting

Thus, ethnography is being taken to include participant observation and is also taken to encapsulate the notion of ethnography as a written product of ethnographic research.

Considering this set of criteria against the colour forecasters' methodological approach to trend forecasting, not only are a number of observations made but also a number of unanswered questions materialise. First of all, we must question whether or not colour forecasters immerse themselves in a setting, a feature which would be required of an ethnographic approach. Given that colour trends are generalised for a more universal colour forecast, it would not be possible to immerse oneself in a single setting with all of the individuals subjected to being observed. It therefore is more likely that the colour forecaster observes many colour clues from outside of the setting hence the idea of a colour forecaster currently undertaking ethnographic research fails on the first of Bryman's criteria. The colour forecaster does however satisfy criterion number two as observing people is an ongoing process. Number three beckons the following questions: Do colour forecasters overtly ask questions of those they observe? Do they otherwise engage in conversations with those they observe? Or, indeed, do colour forecasters covertly listen in on conversations? While many state that interviewing techniques and focus groups are used, this would appear to be by trend forecasters with vested interests in the macro-trends and megatrends, the suggestion that colour forecasters also engage with a similar process appears to be more tenuous. Also, as discussed throughout Section 6, colour and the perception of colour is complex and would not lend itself well to being discussed, rather some form of colour preference or colour acceptance testing would be more beneficial. Similarly, it is unlikely that people within the group under observation are formally interviewed. With regards to number 5 of the above criteria, it is likely that the fashion scanning process, if undertaken, would provide further generalised documentation that may be relevant to the group under observation. However, it cannot be imagined that there is any documentation that would enlighten the colour forecaster regarding colour preferences and colour 
acceptance for the said group, and therefore having nothing to analyse. We must also consider who this 'group' of people are, do colour forecasters focus on a micro-level to observe the colour preferences of small groups of people? The literature would suggest that colour trends emerge from the identification of the reappearance of a colour, or colours from the overall observation process rather than being identified more within cluster groups, though this can happen. Also, colour forecasts are built around a limited number of key fashion colours to create a harmonious colour palette, as discussed in Section 3.2. Also, it is not unknown for colour forecasters to reintroduce a colour that has been off the public radar for a period of time [477]. Finally, the colour forecast itself, with its supporting story, may be considered a bona fide entity to satisfy the seventh criterion. Generally, it can concluded that while some elements of an ethnographic research approach may be able to be credited to macro-trend forecasters, the method cannot be claimed by colour forecasters, at least not using Bryman's criteria.

Silver et al [478] note that modified forms of ethnography are often used in commerce for the purpose of studying consumers. They refer to marketing ethnography, as undertaken by market researchers to 'experience life events', they ask questions and record the observations of their own feelings towards the situations in question, which would appear to be product-related given the examples that they use. One such example discloses that Proctor and Gamble use an ethnographic technique to observe consumption practice, where researchers video participants in their homes over periods of time to capture their behaviour first-hand. While it may be marginally easy to appreciate ethnographical approaches to observing consumer behaviour in the macro-environment where macro-trends and mega-trends emerge slowly, lasting for a considerable period of time and slowly fading away, again it is difficult to comprehend its use as a short-range forecasting method that is demanded currently by the fashion industry. However, as the method is already gaining favour in the business world, and if fashion is successfully deaccelerated, advocates of slow-fashion believe it is not just desirable but necessary for more sustainable consumption practice, then perhaps a new form of ethnography specific to colour forecasting and fashion trend forecasting would be both possible and appropriate in a changing humanbusiness environment. This could potentially better support the circular economy and closed loop practice, and better serve consumers if their colour preferences are indeed met. The need therefore to better understand the Human-business interface relevant to colour forecasting expands this concept in the following Section, and furthermore provides a sound rationale to support a more sustainable colour forecasting model of practice that has been reasoned through the unique critical thinking of current forecasting practice.

\section{The human-business interface}

In Section 7 the mechanisms that facilitate colour and trend forecasting were explored, including the theory of planned obsolescence as a vehicle for promoting change from an industry perspective, all of which are largely for the benefit of industry. Section 8 focused on further analytical tools and analysis techniques demonstrating how the general forecasting system has become overly complex, which may be apportioned to agency differentiation. While forecasting essentially serves the industry in providing apparently near-accurate data analysis of the readiness of consumers for colour and style change, colour forecasts should also serve consumers, as the colours anticipated to be acceptable to them inform the eventual forecast colour palettes for a given season that they are then expected to wear, or at least 
purchase. However, anticipation itself can be fallible and colour forecasting should be more accurately described as being a design and marketing tool used to drive sales volume, rather than being a method of accurately predicting what colours consumers would be more willing to wear given the choice. Furthermore, the Human-Business interface that colour forecasting potentially provides should then ideally have a convergence of consumer colour preferences, which may not necessarily always be visible, and the industry capability to provide these, thus mutually satisfying consumer and industry needs, though in a sustainable manner. Currently colour forecasting provides the means for industry to promote a fallacy of a trend being an accurate reflection of consumer colour preference for a season. In reality colour forecasting provides choices that may be only marginally acceptable in the here and now, as consumers are aware that other colour options will be made available to them in a relatively short timeframe they will buy-in to the trend.

Section 8 also exposes the emphasis on expanding the forecasting system into the macro- and megatrend environment to identify, track and predict cultural shifts. Such data collection and analysis does not appear to, as yet, be applied to colour, and the sophisticated tools used by those forecasters do not appear to have much relevance to colour forecasting. In addition, the concept of globalised colour forecasts that are provided by the larger colour forecasting agencies, such as CAUS and CMG, offer further considerations for the colour forecast researcher. In conducting a wider search for colour clues, the more likely the colour forecaster is to spot the same clues globally. This in itself though provides no guarantee that many like-minded individuals exist in significant numbers within the smaller locations where product in those colours may be offered. In other words, the further afield one looks for the same colour clues the more likelihood of finding them, which then leads to a generalisation of a fact, or the promotion of a distorted fact. This generalisation of colour consensus at a given period of time cannot therefore be successfully localised unless the same colour clues can be significantly identified within that much smaller location. Similarly, mega-trends are given recognition as being global movements, yet how such trends exist within clusters of individuals in more isolated regions may not be sufficiently significant to provide an accurate analysis of what local, regional or even national needs and preferences may be. There is also, perhaps, too much of a suggestion that generalised consumer lifestyles that have some level of global relevance have equal relevance in smaller communities. It must be more important to any company serving a particular segment of the population to be more considerate of local needs and preferences and have a significant understanding of their own clientele, in which case how can it be expected that global forecasts will accurately translate into more localised forecasts; or that the same forecast will appeal to individuals in all locations that the company may serve.

\subsection{Colour preferences}

The literature affirms that using consumer colour preferences 'correctly' can increase the volume of sales, which further promotes the purpose of colour forecasting as a tool for economic purposes and financial stability for the industry. 
For Diane and Cassidy [479] and later Cassidy [480; 481; 482] the use of colour preferences, and a lack of more significant research into colour preferences relative to particular fashionable products was identified as being the weakness of the current colour forecasting system. McLuckie [483] went some way in demonstrating a relative lack of colour knowledge amongst fashion and textile design undergraduates, who were engaging with the colour-forecasting process as a part of their degree courses. Furthermore, there had been an assumption that undergraduates had gained sufficient colour knowledge from art classes at school, before going to University. No evidence was found to support that colour forecasters hold a strong understanding of the colour theories and knowledge of the tools described earlier (Section 6), let alone use them. This beckons the question of how colour forecasters can be expected to work with colour to provide meaningful colour forecasts with relatively little knowledge of the relevant theories.

Colour preferences are complex. Schloss et al [484] report that hue preferences in relation to product have a tendency to vary 'along a blueness-yellowness axis, with a peak at blue and a trough around yellowish-green'. Furthermore, they state that regardless of the 'robust pattern in average preference data, there are [apparent] large individual differences [and that] the origins ... are not entirely understood'. In addition, they acknowledge that, at present, colour preferences 'have yet to be captured fully through a predictive model'. Prior to this, a further complication regarding seasonal colour preferences was identified [485] as colour preference ratings by individuals' appear to have a tendency to change on a seasonal basis. Colour forecasters however may compensate for this, possibly unknowingly, as they tend to promote pastels for the warmer spring/summer forecasts and shades for autumn/winter forecasts in line with natures' seasonal colour changes as discussed in Section 7.

There is a generalisation that colour preferences are ordered in relation to Hans Eysenck's [486] 'Significant Pattern of Colour Aesthetics' study. As previously stated, Eysenck had identified a universal 'common order to colour preference', being blue, red, green, purple, yellow and orange [487]. However, to date, colour preference tests use a very limited selection of colours and invariably include only one example of each hue; the nuances of colour are not tested. It would be beneficial for colour forecasters to provide colour palettes and colour combinations that relate better to consumer colour preferences but In order to do so, a greater understanding of colour preferences in relation to the nuances of colour would be required. Furthermore, as colour preferences may also be linked to changes in colour taste, identifying correlations 'between the swings of individuals' colour taste, the colour zeitgeist and more general colour preferences' could be critical to accurate colour forecasting and product-type. Cassidy [270] provides a framework for working with the nuances of colour through subjective colour mixing principles, as given below, using colour bias (also see Section 6.4.2) as the critical colour nuance factor. Six primary colours are proposed in accordance with Wilcox [488] and Lloyd [489]:

- Red 1 having a blue bias

- Red 2 having a yellow bias

- Yellow 1 having a blue bias

- Yellow 2 having a red bias

- Blue 1 having a red bias 
- Blue 2 having a yellow bias

As a consequence, there are twelve potential secondary colours, four for each of the three secondary colours on a twelve-hue colour wheel (see Section 6.4.1):

- Orange

○ Red $2+$ Yellow 2 = Orange 1 having an orange bias; the purest orange possible

- Red $1+$ Yellow 1 = Orange 2 having a blue bias

○ Red $1+$ Yellow 2 = Orange 3 having a violet bias

- Red $2+$ Yellow $1=$ Orange 4 having a green bias

- Green

- Yellow $1+$ Blue 2 = Green 1 having a green bias; the purest green possible

- Yellow $2+$ Blue $1=$ Green 2 having a red bias

- Yellow $2+$ Blue 2 = Green 3 having an orange bias

- Yellow $1+$ Blue $1=$ Green 4 having a violet bias

- Violet (Purple)

- Blue $1+$ Red 1 = Violet 1 having a violet bias; the purest violet possible

- Blue $2+$ Red $2=$ Violet 2 having a yellow bias

○ Blue $2+\operatorname{Red} 1=$ Violet 3 having a green bias

- Blue $1+$ Red $2=$ Violet 4 having an orange bias

The intermediary hues Red-Orange, Yellow-Orange, Yellow-Green, Blue-Green, Blue-violet and RedViolet of the standard twelve-hue colour wheel have similar varying biases. As there are six potential primary colours and four potential secondary colours there are eight potential variations of each of the six basic intermediary colours. For example, the eight variations of Red-Orange are as follows:

- Red-Orange $1=$ Red $1+$ Orange 1 having a blue-orange bias

- $\quad$ Red-Orange 2 = Red $1+$ Orange 2 having a blue bias

- $\quad$ Red-Orange 3 = Red $1+$ Orange 3 having a blue-violet bias

- Red-Orange 4 = Red $1+$ Orange 4 having a blue-green bias

- Red-Orange $5=$ Red $2+$ Orange 1 having a yellow-orange bias; the purest red-orange possible

- Red-Orange $6=$ Red $2+$ Orange 2 having a green bias

- $\quad$ Red-Orange $7=$ Red $2+$ Orange 3 having a yellow-violet bias

- Red-Orange 8 = Red $2+$ Orange 4 having a yellow-green bias

The underlying idea is that while an individual may have a liking for blue, they will not favour all blue variations depending on the underlying bias, even prior to the complications of adding white, black or grey for different tonal values. Thus far, an in-depth study of colour preferences in relation to this framework has not been undertaken beyond the critical analysis of McLuckie's experiments detailed in Section 6. It would be of interest to see this art/design-based framework applied in the context of textiles because the challenge of being able to assess the degree of colour acceptance for a product at any given time by any segment of a population can be more accurately understood if colour preferences are known. 


\subsection{Colour psychology}

Knowledge of colour psychology should be of benefit to the colour forecaster, as colour is known to actively affect our emotions and as a consequence, our behaviour. Also, slow moving trends such as an individuals' colour taste (see Section 7.4) have deeply rooted emotional dimensions and therefore psychological meaning. Colour psychologists attempt to understand how we are affected by colour, and why the experiences are often different between individuals. To try to better-understand colour preferences, attempts have been made to try to find colour combinations that appeal more broadly to sets of people and to attempt to test those theories [490]. It is this approach that forms the basis of the seasonal colour palettes associated with individuals as described in Sections 6.5 and 7. While it is widely accepted that colour can be used to great effect to communicate visually to an audience, and the importance of symbolic associations of colour are indicated by authors of colour forecasting, there is a lack of in-depth research and therefore knowledge of how colour psychology might be used in colour forecasting. The general guidelines that are widely accepted are used extensively in marketing efforts such as in packaging and branding and in the creation of attractive environments such as hotel lobbies and restaurants [491]. Yet, the challenge in trying to make such generalisations meaningful as a colour forecasting tool appears to be limited at present, largely because the positive associations relate less to products such as clothing. Individuals appear to be more influenced by colours they feel suit them, that they feel comfortable in, that they feel sends out the correct message about them, or simply because others around them are wearing it, in other words, they are following a trend. There is possibly a fine line between colour preferences for particular products, such as skirts, jackets, jumpers/jerseys, and the underlying colour psychology for this choice; the what and the why. For the colour forecaster, is the 'why' so necessary to understand?

\subsection{Cultural colour meaning}

The meaning of colour, or more specifically, colour associations, particularly between cultures, should also be given due consideration in relation to colour forecasting. Where colour psychology may be viewed as being an innate factor that is personal and therefore different between individuals, even from similar backgrounds and upbringing, cultural colour meanings are often collectively shared amongst families, communities, regions and countries. As Gans [492] recognised, cultural contexts can influence preferences (see Section 8.2) and therefore it may be possible to make better generalisations about colour preferences in relation to cultural beliefs and norms than it is to generalise within the context of colour psychology. There are known references to cultural meanings of particular colours that are often used within customs and in language. For example, red is symbolic of good luck in China, the red rose in Ancient Greece and Rome was sacred to Aphrodite, which has transcended time; in the West it is associated with love and passion [493]. While there are some colour associations that are more general, such as feeling blue, or being green with envy, largely, colour associations are attached to items, such as the red rose example. Incidentally, different coloured roses have different meanings, a yellow rose is generally associated with friendship and good health, but yellow roses may be given to friends to commemorate the loss of a loved one. As each colour essentially holds both positive and negative connotations, it is difficult to create guidelines for colour use in clothing across cultures. In addition, research into colour preferences specific to product-types, even in isolation of one culture, is severely 
lacking. However, subcultures who adopt a certain style of dress, which often incorporates the use of particular colours may present colour forecasters with some insight into colour preferences for a specific group of individuals, though this would not necessarily work as a colour palette for other groups of individuals outside of the same subculture genre. Such colour inspirations may already have been drawn upon by the forecaster for inspiration for a more general colour palette.

Therefore, while colour psychology and cultural colour meanings may provide points of inspiration for colour direction for design and colour forecasting, or at least as areas of interest to explore, in reality such knowledge may more likely be put to better use in selling the colour theme to an audience as discussed throughout Section 5. However, there is still some value to be considered in terms of physical locations and localised colour forecasts that are discussed in Section 10, where a detailed understanding of colour preferences would be of extreme benefit, the cultural meaning of colour may then also be of benefit and colour psychology may play a relatively small part to give added value to more localised colour palettes and forecasts. Currently, colour forecasts from the large agencies such as the Color Association of the United States (CAUS) and the Color Marketing Group (CMG), are intended for a global clientele without any apparent consideration of the cultural element. It is assumed that the designers who use such forecast information will have their own understanding of the culture of their consumers and will consequently adjust the colour palettes to suit their own needs. It could be argued that local colour forecasts could be of more value to the industry than the non-culturally focused colour stories that are commonly disseminated.

\section{Colour forecasting in the twenty-first century}

The current colour forecasting system can be considered to contribute in a negative way to the carbon footprint of the fashion industry hence the interest in how the system might be improved to benefit not just the stakeholders but also the environment. It revisits the concept of an ethnographic approach to colour forecasting and builds upon the concepts of the newly termed colour zeitgeist (see Section 3.5) and colour-design scanning and analysis (see Section 8.2), and links back to colour preferences, colour taste and colour cycles though within the context of localised colour palettes and forecasts. It considers the colour forecaster as a Master of their own locality and how this approach may allow for the individuality that people may seek in their colour choices, thus personalising colour in a way that the global colour forecasters could never achieve.

\subsection{Unsustainable practice}

While colour forecasting is not wholly responsible for the issue of global textile waste, it does play its part along with general trend forecasting in developing a process of planned obsolescence to drive change and as a consequence promote sales. It is difficult therefore to divorce trend forecasting from excessive consumer consumption of clothing and the impact of this on the environment. In order to slow fashion down, trends must also slow down and this poses the question: If the clothing we purchase really resonates with our colour taste and preferences, would we really feel the need to purchase so frequently and what, in theory would appear to be a more responsible approach to colour forecasting for the twenty-first century. 
It is however important here to situate colour forecasting within the context of the circular economy and within the textile waste conundrum. The Ellen MacArthur A new textile economy: Redesigning fashion's future report sets out the main drivers of unsustainable practice and the ideals for a more sustainable textile industry. The report states that the driving force behind the sustainability challenge resides in the 'increased per capita sales in mature economies' driven mainly by fast fashion with its 'quicker turnaround of styles, increased number of collections offered per year and often at low prices' which is linked to the demands of a global 'middle-class population'. In addition to the challenges that the textile industry face, such as reducing water pollution and the use of hazardous substances and improving the working environment, extending the life of garments and textiles has become a very important problem to solve. With less than $1 \%$ of textile waste re-entering the cycle through its inclusion into new materials for fashion, and clothing being 'massively underutilised after purchase, a systemic solutions are being sought. Colour forecasting and trend forecasting promote product change to generate sales and have contributed to the speed of change and excessive consumption [494]. By changing the colour forecasting process to promote slow change, and having a better understanding and employment of colour-preference data and cultural colour-acceptance knowledge could potentially ensure that fashion could be more sustainably consumed and valued by consumers through their emotional attachment to its colour. To be effective, such an approach would require collaboration between colour forecasters, the fashion and textile industry (dyers and colourists, designers, buyers, retailers etc.) and consumers.

\subsection{Improving the colour forecasting approach}

Returning to Bryman's guidelines [495] given in Section 8.5 to reconsider a potential ethnographic approach to colour forecasting, it would be necessary in the first instance, for the colour forecaster to be 'immersed in the social setting for an extended period of time'. Rather than focussing on travelling around the globe looking for a consensus of colour clues, the colour forecaster working within a singular environment can observe the organic changes in that setting that would essentially slow-down the urgency to recreate colour trends at speed. Colour forecasters would become experts in their chosen locations to better inform the practitioners and industry personnel who serve those communities and become more-sensitised to their communities and therefore provide more meaningful colour data for them. Being located within the social setting on a more permanent basis would allow for 'regular observations' and would facilitate opportunities to engage in conversations that Bryman deems essential and would allow, not only for an in-depth recording and analysis of the culture and the preferences, but also would allow for experimentation within the environment. Many colour forecasters begin their academic life as designers; for those with a real interest in colour, being in situ, would allow them to take a hands-on approach to colour experimentation and colour palette creation; tools such as photography, other forms of artwork and craft engagement could be incorporated into the colourforecasting system as time could be afforded to do so.

\subsection{Summary and Conclusion}

This issue of Textile Progress has focussed on analysis of the literature, the processes, the rationales and primary research data about colour forecasting. Clearly there is a need for more in-depth studies to 
provide detailed analyses into a number of areas that can further expose the underlying truth and provide new insights. Much of the current literature reviewed is based on secondary research with added interview data from professionals but offers little in the way of critical analysis, any deliberation of the weaknesses, nor any suggestions for improvement. There is a clear need to simplify colour forecasting, to concentrate on providing consumers with meaningful colour choices, and to develop a stronger sustainable emphasis for colour forecasting. In order for this to happen the ethos of colour forecasting must change and more serious attempts to truly track the evolution and cyclic nature of colour taste through a colour zeitgeist, and possibly through an ethnographic approach to colour forecasting are considered to be possible avenues by which this may occur. The need for a twenty-first century approach to create a more sustainable practice is indicated.

Colour forecasting has developed over the last century into a significant component of the fashion and textiles industry employing a variety of processes, tools and techniques to provide colour direction that filters throughout the design, manufacturing, retailing and marketing sectors of industry. As new companies have joined this ever-growing sector, colour forecasting itself has grown to become more complex and companies have developed their own niche(s). Increased global competition in a saturated fashion and textiles marketplace has provided the opportunity for colour forecasting and trend forecasting to become considered increasingly-important in ensuring the hoped-for level of sales of fashion products because of their potential to accelerate the rate of trend-change through planned obsolescence. As a consequence, the current colour-forecasting process and the more general fashiontrend-forecasting processes add significantly to the textile waste conundrum through their encouragement of fast-paced change that helps drive excessive fashion consumption. A radical systemchange is now imperative for colour-forecasting processes to become sustainable, to significantly contribute to the circular economy and thus to deliver on twenty-first century needs, for the economy, for society, and for the environment.

Slowing down the rate of change is one method that would need to be employed by forecasters. Rather than continuing with the $20^{\text {th }}$ century philosophy of colour being just a powerful sales tool for sales volume creation, and thus very much a marketing tool, it would be more beneficial for the future for forecasters to view colour as being capable of taking on a new role and become a powerful sustainability / circular economy tool. What is put forward here therefore is the concept of designing forecasts rather than simply creating them. In order to achieve this radical change, the human-business interface needs to be at the forefront of the process, potentially with considerations of colour psychology, but more particular with the inclusion of consumer colour-preference data in relation to cultures, or colour typologies, and the cultural meaning of colours to ensure that products better reflect consumer taste.

The reinforcement of emotional attachment to products through their colour would encourage consumers to use products for longer, though this needs to be supported by durability of materials and product, and through high-value creation through design. There is a necessity to more-thoroughly understand and work with patterns in consumer behaviour and acceptance of colour. This links directly with the 'colour zeitgeist'. With a more transparent colour zeitgeist the anticipation of consumer acceptance of colour trends would essentially be eliminated; the anticipation of consumer acceptance is after all a difficult parameter of the forecasting process for forecasters to otherwise overcome. A more- 
substantial body of consumer colour preference data is required in order to discover the natural colour order of preferences among cultures. In-depth enquiries into the subtle, and more obvious changes in preference in relation to colour nuance is also required to further validate the colour zeitgeist within present and future contexts.

Traditionally marketing efforts, such as trade fairs and the media have worked to disseminate and reinforce trends. More recently new technologies have opened opportunities for new channels and leaders to direct trends, such bloggers. Forecasters are now experiencing competition from outside of the sector as well as from within. Such interventions may further erode the necessary control needed to create a sustainable system and practice, as the pace of change could be increased to catastrophic levels. A way to harness control, and thus slow down trend-change would be to develop a system that relies less on taking inspiration from the present to project into the future, and more on the use of tools such as the fashion pendulum and the product lifecycle models to carefully and more deliberately design colour forecasts. Theme building prior to creating colour stories can facilitate this. Themes that reflect the moods of cultures, or the mega-trends that are identified within the socio-economic environment and in the colour zeitgeist could be used to anchor slow colour change within longer-lasting themes. To further establish this, perhaps an emphasis should be placed on slowing down the fast-pace of creating forecasts through the design tools, such as mood boards and sketchbooks, in the more traditional manual way; and either a move away from a reliance on the faster-paced capabilities of digital technologies, or even to develop slow-paced digital technologies.

A further consideration is the usefulness of groups of people being involved in the forecasting process driven by colour meetings. Invariably used to direct a consensus of colour by fusing the different viewpoints of the meeting members to create agreeable colour palettes may render colour forecasts not only globally-uniform but also bland and reactionary. If individual forecasters created forecasts for smaller groups of clients, the forecasts may become more revolutionary in relation to cultural-consumer colour preferences and create a better choice for consumers and encourage longevity of garments in the consumption lifecycle. More generally, the role of colour forecasting could be developed to help create stronger relationships between the designers and retailers and colourists to achieve sustainability benefits; this concept is worthy of a considerable in-depth investigation.

\section{References}

1. The Encyclopedia of Greater Philadelphia. Rutgers University sOn-line]. 2018. [Accessed]. Available from: https://philadelphiaencyclopedia.org/archive/textile-manufacturing-and-textile-workers/.

2. Valan, F. The evolution of colour in design from the 1950s to today, Journal of the international Colour Association. (2012), 8, 55-60.

3. Ellacott, S.E. Spinning and weaving. Methuen \& Co, Ltd. London, 1956.

4. Rouse, E. Understanding fashion. BSP Professional Books Oxford, 1989.

5. Lehnert, G. Fashion: A concise history. Laurence King Publishers London, 1999. 
6. Bradfield, N. Costume in detail 1730 - 1930. Harrap Ltd. England, 1985.

7. Baudot, F. A century of fashion. Thames \& Hudson Ltd. London, 1999.

8. Callan, G.O. The Thames \& Hudson dictionary of fashion and fashion designers. Thames \& Hudson London, 1998.

9. Forster, S.V. and Christie, R. The significance of the introduction of synthetic dyes in the mid-19 ${ }^{\text {th }}$ century on the democratisation of Western fashion. International Colour Association. 2013, 11, pp.1-17.

10. Marcketti, S.B. and Parsons, J.L. American fashions for American women: Early $20^{\text {th }}$ century efforts to develop an American fashion identity. Dress. 2007, 34, pp.79-95.

11. The Color Association of the United States. [On-line]. 2019. [Accessed]. Available from: https://en.wikipedia.org/wiki/Color Association of the United States

12. Burns, L.D. and Bryant, N. The business of fashion. Fairchild's Publications New York, 1997.

13. Revolvy. The Color Association of the United States. [On-line]. 2019. [Accessed]. Available from: https://www.revolvy.com/page/The-Color-Association-of-the-United-States

14. The Color Association of the United States cited in Kim, E. et al. Fashion trends analysis and forecasting. Berg London, 2011, pp.73.

15. Walch, M. Foreword. In: Colour consulting - A survey of international colour design. Van Nostrand Reinhold New Jersey, 1994, pp.viii-x.

16. Blaszczyk, R.L. and Wubs, B. The fashion forecasters. Bloomsbury London, 2018, pp.1.

17. Callan, G.O. The Thames \& Hudson dictionary of fashion and fashion designers. Thames \& Hudson London, 1998.

18. Burns, L.D. and Bryant, N. The business of fashion. Fairchild's Publications New York, 1997.

19. Mendes, V. and de la Haye, A. Twentieth century fashion. Thames \& Hudson London, 1999.

20. Worth, G. The lineage of colour forecasting in the UK. Point. 2000, 9.

21. Sizing up America: Signs of expansion from head to toe. [On-line]. 2004. [Accessed]. Available from: https://www.nytimes.com/2004/03/01/us/sizing-up-america-signs-of-expansion-from-head-to-toe.html

22. Wubs, B. Interstoff's fashion table. In: The fashion forecasters. Bloomsbury London, 2018, pp.167190.

23. Rouse, E. Understanding fashion. BSP Professional Books Oxford, 1989.

24. Donzé, P.Y. Fashion prediction and the transformation of the Japanese textile industry. In: The fashion forecasters. Bloomsbury London, 2018, pp.149-165. 
25. Giertz-Mårtenson, I. Looking behind the scenes of Swedish fashion forecasting. In: The fashion forecasters. Bloomsbury London, 2018, pp.213-233.

26. The color tribe speaks. [On-line]. 2019. [Accessed]. Available from: https://colormarketing.org/2018/08/23/color-design-steering/

27. McKelvey, K. and Munslow, J. Fashion forecasting. Wiley-Blackwell Oxford, 2008.

28. The midi skirt, divider of nations. [On-line]. 2014. [Accessed]. Available from: https://www.theatlantic.com/entertainment/archive/2014/09/the-return-of-the-midi-skirt/379543/

29. Colour forecasting: an investigation into how its development and use impacts on accuracy. [On-line]. 2011. [Accessed]. Available from: file:///G:/Textile\%20Progress/Revised\%202019/Thesis King.pdf

30. Timeline: The history of haute couture. [On-line]. 2019. [Accessed]. Available from: https://eluxemagazine.com/magazine/the-history-of-haute-couture/

31. Gaimster, J. The changing landscape of fashion forecasting. Fashion Design, Technology and Education. 2012, 5(3), pp.169-178.

32. Cassidy, T.D. Consumer colour and style preferences: A new approach to tackling the textile waste conundrum. In: Eco-friendly and fair. Routledge Oxon UK, 2018, pp.83-91.

33. Cassidy, T.D., and Cassidy, T. Using soft systems methodology to improve the colour forecasting process. Journal of the International Colour Association. 2012, 7, pp.27-50.

34. Whiteley, N. Toward a throw-away culture: Consumerism, style obsolescence and cultural theory in the 1950s and 1960s. Oxford Art Journal 1987, 10(2), pp.3-27.

35. Sproles, G.B. and Burns, L.D. Changing appearances: Understanding dress in contemporary society. Fairchild New York, 1994.

36. Craik, J. Fashion: The key concepts. Berg Oxford, 2009, pp.213.

37. Frings, G.S. Fashion: From concept to consumer. Pearson Essex, England, 2008.

38. Jackson, T. and Shaw, D. Mastering fashion buying and merchandising management. Palgrave Hampshire, England, 2001.

39. Brannon, E.L. Fashion forecasting. $3^{\text {rd }}$ edition. Fairchild New York, 2010.

40. Dillon, S. The fundamentals of fashion management. A\&C Black London, 2011, pp.84.

41. Secrets of signature colour. [On-line]. 2008. [Accessed]. Available from: http://www.pointeradvertising.com/creative brief/12 06 design tip.html

42. Eckman, M. et al. Toward a model of the in-store purchase decision process: Consumer use of criteria for evaluating women's apparel. Clothing and Textiles Research Journal. 1990, 8(2), pp.13-22. 
43. How brands use colour psychology to reinforce their identities. [On-line]. 2019. [Accessed]. Available from: https://econsultancy.com/how-brands-use-colour-psychology-to-reinforce-their-identities/

44. Ten colours that increase sales, and why. [On-line]. 2013. [Accessed]. Available from: https://www.business2community.com

45. Churchill, G.A. and Lacobucci, D. Market research: Methodological foundations. Earlie Lite Books Nashville, 2005.

46. Bye, E. Fashion design. Berg Oxford, 2010, pp.46.

47. Brannon, E.L. Designer's guide to fashion apparel. Fairchild New York, 2011, pp.5.

48. Cadigan, E. Sourcing and selecting textiles for fashion. Bloomsbury London, 2014.

49. Calderin, J. The fashion design reference and specification book. Rockport Publications Massachusetts, 2013, pp.52.

50. Business forecasting using historical data and regression analysis. [On-line]. 2019. [Accessed]. Available from: https://www.smallbusiness.chron.com

51. Fletcher, K. and Tham, M. Clothing rhythms. In: Eternally yours: Time in design. 010 Publishers Rotterdam, 2004, pp.254-274.

52.Cline, E. Over-dressed: The shockingly high cost of cheap fashion. Penguin New York, 2012, pp. 103.

53. Four ways brands can use the psychology of colour as a competitive edge. [On-line]. 2017. [Accessed]. Available from: https://www.adweek.com/digital/peter-trebek-go-transcript-guest-postpsychology-of-color/

54. Eysenck, H.J. Type-factors in aesthetic judgements. British Journal of Psychology. 1941, 31(3), pp.262-270.

55. Porter, T. Color in the looking glass. In: Colour consulting - A survey of international colour design. Van Nostrand Reinhold New Jersey, 1994, pp.1-9.

56. Whitfield, T.W. and Whelton, J. The arcane roots of colour psychology, chromatherapy and colour forecasting. Colour Research \& Application. 2015, 40(1) pp.99-106.

57. Diamond, J. and Diamond, E. (2013). The world of fashion. $5^{\text {th }}$ edition. Fairchild New York, 2013, pp.19.

58. Diamond, J. and Diamond, E. (2013). The world of fashion. $5^{\text {th }}$ edition. Fairchild New York, 2013, pp.431.

59. Stijlinstituut. [On-line]. 2019. [Accessed]. Available from: www.stijlinstituut.nl 
60. Tan, J. Colour hunting: How colour influences what we buy, make and feel. Frame Amsterdam, 2011, pp.40.

61. Linton, H. (1994). Colour consulting - A survey of international colour design. Van Nostrand Reinhold New Jersey, 1994.

62. Rousso, C. Fashion forward: A guide to fashion forecasting. Fairchild Canada, 2012, pp.7.

63. Scully, K. and Cobb, D.J. Colour forecasting for fashion: Portfolio skills. Laurence King London, 2012, pp.7.

64. Worth Global Style Network. [On-line]. 2019. [Accessed]. Available from:

https://www.wgsn.com/en/wgsn/

65. Do fashion trends still exist? [On-line]. 2015. [Accessed]. Available from:

https://www.businessoffashion.com/articles/intelligence/fashion-trends-still-exist

66. Tan, J. Colour hunting: How colour influences what we buy, make and feel. Frame Amsterdam, 2011, pp.40.

67. Raymond, M. The trend forecaster's handbook. Laurence King London, 2010, pp.14-15.

68. Kim, E. et al. Fashion trends analysis and forecasting. Berg London, 2011, pp.xiii.

69. McKelvey, K. and Munslow, J. Fashion forecasting. Wiley-Blackwell Oxford, 2008, pp.1.

70. Rousso, C. Fashion forward: A guide to fashion forecasting. Fairchild Canada, 2012, pp.184.

71. Diamond, J., and Diamond, E. The world of fashion. Fairchild New York, 1997.

72. Healey, K.R. An analysis of forecasting and fashion services available for knitwear manufacturers. Knitting International. 1984. 91(1091).

73. Healey, K.R. An analysis of forecasting and fashion services available for knitwear manufacturers. Knitting International. 1984. 91(1092).

74. Healey, K.R. An analysis of forecasting and fashion services available for knitwear manufacturers. Knitting International. 1985. 92(1093).

75. Healey, K.R. An analysis of forecasting and fashion services available for knitwear manufacturers. Knitting International. 1985. 92(1094).

76. Healey, K.R. An analysis of forecasting and fashion services available for knitwear manufacturers. Knitting International. 1985. 92(1095).

77. Healey, K.R. An analysis of forecasting and fashion services available for knitwear manufacturers. Knitting International. 1985. 92(1096). 
78. Zessler, I. Peclers Paris. In: Colour consulting - A survey of international colour design. Van Nostrand Reinhold New Jersey, 1994, pp.195-202.

79. Brannon, E.L. Fashion forecasting. Fairchild New York, 2000, pp.25.

80. Brannon, E.L. and Divita, L. Fashion forecasting. $4^{\text {th }}$ edition. Bloomsbury New York, 2015, pp.9.

81. Danger, E.P. The colour handbook: How to use colour in commerce and industry. Gower Aldershot UK, 1987.

82. Rousso, C. Fashion forward: A guide to fashion forecasting. Fairchild Canada, 2012, pp.25.

83. Brannon, E.L. and Divita, L. Fashion forecasting. $4^{\text {th }}$ edition. Bloomsbury New York, 2015, pp.6-9.

84. Rousso, C. Fashion forward: A guide to fashion forecasting. Fairchild Canada, 2012, pp.8.

85. Fiore, A.M. and Kimle, P.A. Understanding aesthetics for the merchandising and design professional. Fairchild New York, 1997, pp.379.

86. Edelkoort. World review of textile design. International Textiles. 1991, 725.

87. McKelvey, K. and Munslow, J. Fashion forecasting. Wiley-Blackwell Oxford, 2008, pp.102.

88. We connect fashion. [On-line] 2019. [Accessed]. Available from:

https://www.weconnectfashion.com/businesses/trend-union

89. Edelkoort, L. The theories behind colour forecasting. The Briggait Centre, Glasgow. $19^{\text {th }}$ October, 1999 (Presentation).

90. Cassidy, T.D. and Kettley, S. Fashion design. In: The Bloomsbury Encyclopedia of Design. Bloomsbury London, 2015, Vol 2. pp.5-11.

91. Davis, M. L. Visual design in dress. Second edition. Prentice-Hall New Jersey, 1980.

92. Cassidy, T.D. and Kettley, S. Fashion design. In: The Bloomsbury Encyclopedia of Design. Bloomsbury London, 2015, Vol 2. pp.5-11.

93. Diane, T. and Cassidy, T. Colour forecasting. Blackwell Oxon UK, 2005, pp.106-7.

94. Tham, M. Lucky people forecast - A system futures perspective on fashion and sustainability. [Online] 2008, pp.36. [Accessed]. Available from: http://research.gold.ac.uk/11301/

95. McKelvey, K. and Munslow, J. Fashion forecasting. Wiley-Blackwell Oxford, 2008, pp.129.

96. Montgomery, J. History, resource, and inspiration. In: Colour consulting - A survey of international colour design. Van Nostrand Reinhold New Jersey, 1994, pp.12-18. 
97. Tan, J. Colour hunting: How colour influences what we buy, make and feel. Frame Amsterdam, 2011, pp.41.

98. McKelvey, K. and Munslow, J. Fashion forecasting. Wiley-Blackwell Oxford, 2008, pp.88.

99. Tan, J. Colour hunting: How colour influences what we buy, make and feel. Frame Amsterdam, 2011, pp.44.

100. McKelvey, K. and Munslow, J. Fashion forecasting. Wiley-Blackwell Oxford, 2008, pp.88.

101. Scully, K. and Cobb, D.J. Colour forecasting for fashion: Portfolio skills. Laurence King London, 2012, pp.24.

102. Tan, J. Colour hunting: How colour influences what we buy, make and feel. Frame Amsterdam, 2011, pp.41.

103. Dee, J. and Taylor, L. Colour therapy: The symbolism, use and healing effects of colour. Silverdale Leicester UK, 2002, pp.6.

104. Danger, E.P. Using colour to sell. Gower Aldershot UK, 1968, pp.26.

105. Tate, S.L. Inside fashion design. $2^{\text {nd }}$ edition. Harper and Row New York, 1984.

106. Rousso, C. Fashion forward: A guide to fashion forecasting. Fairchild Canada, 2012, pp.195.

107. Cassidy, T.D. Sustainable colour forecasting. In: Sustainability in Fashion and Textiles. Greenleaf Sheffield UK, 2013, pp.111-124.

108. Rousso, C. Fashion forward: A guide to fashion forecasting. Fairchild Canada, 2012, pp.166-179.

109. Rousso, C. Fashion forward: A guide to fashion forecasting. Fairchild Canada, 2012, pp.171-178.

110. Rousso, C. Fashion forward: A guide to fashion forecasting. Fairchild Canada, 2012, pp.195-200.

111. Diane, T. and Cassidy, T. Colour forecasting. Blackwell Oxford UK, 2005, pp.92.

112. Sproles, G.B. Fashion. Consumer behaviour towards dress. Burgess Minneapolis, 1979.

113. Sproles, G.B. and Burns, L.D. Changing appearances: Understanding dress in contemporary society. Fairchild New York, 1994.

114. Sproles, G.B. Fashion. Consumer behaviour towards dress. Burgess Minneapolis, 1979.

115. Long-term vs. short-term forecasting for the apparel forecasting process. [Online]. 2019. [Accessed]. Available from: https://www.smallbusiness.chron.com

116. Sproles, G.B. Fashion. Consumer behaviour towards dress. Burgess Minneapolis, 1979. 
117. Long-term vs. short-term forecasting for the apparel forecasting process. [Online]. 2019. [Accessed]. Available from: https://smallbusiness.chron.com/longterm-vs-shortterm-forecasting-apparelforecasting-process-35753.html 19/02/2019

118. Brannon, E.L. and Divita, L. Fashion forecasting. $4^{\text {th }}$ edition. Bloomsbury New York, 2015, pp.100. 119. How to fashion forecast. [On-line]. 2019. [Accessed]. Available from: https://www.bl.uk-articleshow-to-fashion-forecast

120. How to choose the right forecasting technique. [On-line]. 2019. [Accessed]. Available from: https://hbr.org-1971/07-how-to-choose-the-right-forecasting-technique

121. The future of fashion forecasting. [On-line]. 2015. [Accessed]. Available from: https://www.notjustalabel.com

122. Mahmood, E. Accuracy in forecasting: A survey. Journal of Forecasting. 3(2), pp.139-159.

123. Sproles, G.B. Fashion. Consumer behaviour towards dress. Burgess Minneapolis, 1979.

124. Brannon, E.L. Fashion forecasting. Fairchild New York, 2000, pp.337-338.

125. Wicked problem. [On-line]. 2019. [Accessed]. Available from:

https://en.wikipedia.org/wiki/Wicked problem

126. Leadership skills: Know the difference between wicked and tame problems. [On-line]. 2019.

[Accessed]. Available from: https://www.christopheravery.com/blog/leadership-skills-know-thedifference-between-wicked-and-tame-problems/

127. Tan, J. Colour hunting: How colour influences what we buy, make and feel. Frame Amsterdam, 2011, pp.42.

128. Keiser, S.J. and Garner, M. B. Beyond design. Fairchild New York, 2003.

129. Brannon, E.L. and Divita, L. Fashion forecasting. $4^{\text {th }}$ edition. Bloomsbury New York, 2015, pp.12.

130. Tan, J. Colour hunting: How colour influences what we buy, make and feel. Frame Amsterdam, 2011, pp.40.

131. Scully, K. and Cobb, D.J. Colour forecasting for fashion: Portfolio skills. Laurence King London, 2012, pp.108.

132. Tan, J. Colour hunting: How colour influences what we buy, make and feel. Frame Amsterdam, 2011, pp.42.

133. Walch, M. Foreword. In: Colour consulting - A survey of international colour design. Van Nostrand Reinhold New Jersey, 1994, pp.viii-x.

134. Brannon, E.L. and Divita, L. Fashion forecasting. $4^{\text {th }}$ edition. Bloomsbury New York, 2015, pp.166. 
135. Scully, K. and Cobb, D.J. Colour forecasting for fashion: Portfolio skills. Laurence King London, 2012, pp.21.

136. Whitfield, T.W. and Whelton, J. The arcane roots of colour psychology, chromatherapy and colour forecasting. Colour Research \& Application. 2015, 40(1) pp.99-106, pp.103-4.

137. Gale, C. and Kaur, J. The textile book. Berg Oxford UK, 2002, pp.136.

138. Porter, T. Color in the looking glass. In: Colour consulting - A survey of international colour design. Van Nostrand Reinhold New Jersey, 1994, pp.1-9.

139. Holland, G. and Jones, R. Fashion trend forecasting. Lawrence King London, 2017, pp.120-122.

140. Verlodt, P. Color marketing group. In: Colour consulting - A survey of international colour design. Van Nostrand Reinhold New Jersey, 1994, pp.32-35.

141. Hulse, T. The colour conspiracy. Hotline Magazine Birmingham UK, 1997.

142. Tan, J. Colour hunting: How colour influences what we buy, make and feel. Frame Amsterdam, 2011, pp.40.

143. Tan, J. Colour hunting: How colour influences what we buy, make and feel. Frame Amsterdam, 2011, pp.42-43.

144. Porter, T. Color in the looking glass. In: Colour consulting - A survey of international colour design. Van Nostrand Reinhold New Jersey, 1994, pp.1-9.

145. Holland, G. and Jones, R. Fashion trend forecasting. Lawrence King London, 2017, pp.122-123.

146. Granger, M. Fashion. The industry and its careers. Fairchild New York, 2007.

147. Jackson, T. The process of fashion trend development leading to a season. In Fashion marketing: Contemporary issues. Butterworth Heinemann Oxford, 2001.

148. Frings, G.S. Fashion concept to consumer. 9 $^{\text {th }}$ Edition. Pearson Essex, 2013.

149. Diane, T. and Cassidy, T. Colour forecasting. Blackwell Oxford UK, 2005.

150. Expofil. [On-line]. 2019. [Accessed]. Available from: https://10times.com/expofil

151. Jackson, T. The process of fashion trend development leading to a season. In Fashion marketing: Contemporary issues. Butterworth Heinemann Oxford, 2001.

152. Kim, E. et al. Fashion trends analysis and forecasting. Berg London, 2011, pp.49.

153. Brannon E.L. Fashion Forecasting. $3^{\text {rd }}$ ed. Fairchild New York, 2010.

154. Kim, E. et al. Fashion trends analysis and forecasting. Berg London, 2011, pp.49. 
155. Michaud, H.A. Intergrated forecasting, scheduling, and planning: Generic model. Wilmington DE, 1989.

156. Brannon, E.L. and Divita, L. Fashion forecasting. $4^{\text {th }}$ edition. Bloomsbury New York, 2015, pp.2.

157. Scully, K. and Cobb, D.J. Colour forecasting for fashion: Portfolio skills. Laurence King London, 2012, pp.32-3.

158. Anniss, E. More fashion labels see the benefits of do-it-yourself. New York Times. December $8^{\text {th }}$ 2008.

159. Brannon E.L. Fashion Forecasting. $3^{\text {rd }}$ ed. Fairchild New York, 2010.

160. Kim, E. et al. Fashion trends analysis and forecasting. Berg London, 2011, pp.154.

161. Edelkoort, L. The theories behind colour forecasting. The Briggait Centre, Glasgow. $19^{\text {th }}$ October, 1999 (Presentation).

162. McKelvey, K. and Munslow, J. Fashion forecasting. Wiley-Blackwell Oxford, 2008, pp.88.

163. Linton, H. Colour consulting - A survey of international colour design. Van Nostrand Reinhold New Jersey, 1994.

164. Hallett, C. and Johnston, A. Fabric for fashion: The complete guide natural and man-made fibres. Lawrence King London, 2014.

165. Wubs, B. Interstoff's fashion table. In: The fashion forecasters. Bloomsbury London, 2018, pp.167190.

166. Wub, B. and Maillet, T. Building competing fashion textile fairs in Europe, 1970-2010: Premiere Vision (Paris) vs. Interstoff (Frankfurt). Journal of Macromarketing. 2017, 37(1), pp.25-39.

167. Behind the scenes at Premiere Vision. The story unfolds. International Textiles 1992, 731.

168. Lidewij Edelkoort. [On-line]. 2017. [Assessed]. Available from: https://www.edelkoort.com/

169. Behind the scenes at Premiere Vision. The story unfolds. International Textiles 1992, 731.

170. Keiser, S.J. and Garner, M. B. Beyond design. Fairchild New York, 2003.

171. Verlodt, P. Color marketing group. In: Colour consulting - A survey of international colour design. Van Nostrand Reinhold New Jersey, 1994, pp.32-35, pp.35.

172. Keiser, S.J. and Garner, M. B. Beyond design. Fairchild New York, 2003.

173. The impact of visual intelligence on trend forecasting. [On-line]. 2018. [Accessed]. Available from: https://medium.com-the-impact-of-visual-intelligence-on-trend-forecasting 
174. Knight, J. Blog marketing: The revolutionary new way to increase sales, build your brand, and get exceptional results. McGraw-Hill Columbus OH, 2006, pp.xi.

175. Holland, G. and Jones, R. Fashion trend forecasting. Lawrence King London, 2017, pp.68.

176. The influence of fashion blogs on consumers. [On-line]. 2019. [Accessed]. Available from: https://marketing.conference-services.net

177. Knight, J. Blog marketing: The revolutionary new way to increase sales, build your brand, and get exceptional results. McGraw-Hill Columbus OH, 2006, pp.281.

178. Solomon, M.R. and Rabolt, N. J. Consumer behaviour in fashion. Pearson Prentice Hall New Jersey, 2009.

179. Holland, G. and Jones, R. Fashion trend forecasting. Lawrence King London, 2017, pp.68.

180. Knight, J. Blog marketing: The revolutionary new way to increase sales, build your brand, and get exceptional results. McGraw-Hill Columbus OH, 2006, pp.94-112.

181. Blog This! [On-line]. 2019. [Accessed]. Available from: https://gta.fandom.com/wiki/...Blog This!

182. Tumblr. [On-line]. 2019. [Accessed]. Available from: https://www.tumblr.com

183. Holland, G. and Jones, R. Fashion trend forecasting. Lawrence King London, 2017, pp.34.

184. Instagram. [On-line]. 2019. [Accessed]. Available from: https://www.instagram.com

185. Pinterest. [On-line]. 2019. [Accessed]. Available from: https://www.pinterest.co.uk

186. Quartermaster Trends. [On-line]. 2019. [Accessed]. Available from: www.quartermastertrends.com

187. Worth Global Style Network. [On-line]. 2019. [Accessed]. Available from: https://www.wgsn.com

188. Holland, G. and Jones, R. Fashion trend forecasting. Lawrence King London, 2017, pp.39.

189. Trendstop. [On-line]. 2019. [Accessed]. Available from: https://www.trendstop.com

190. Style Platform. [On-line]. 2019. [Accessed]. Available from: https://www.styleplatform.com

191. Luminary. Global Colour and Trend Forecasting. [On-line]. 2019. [Accessed]. Available from: https://www.luminarycolour.com

192. Pancel, S., and Cassidy, T.D. The fashion blogosphere: Success from the reader's perspective. Fashion Style \& Popular Culture. 2018, 5(2), pp.246-260.

193. Frings, G.S. Fashion: From concept to consumer. Pearson Essex, England, 2008.

194. Donegar. [On-line]. 2019. [Accessed]. Available from: https://www.donegar.com 
195. Jarnow, J. and Dickerson, K.G. Inside the fashion business. $6^{\text {th }}$ edition. Prentice-Hall New Jersey, 1997.

196. Carlin. [On-line]. 2019. [Accessed]. Available from: https://www.carlin-creative.com

197. Faith Popcorn. [On-line]. 2019. [Accessed]. Available from: https://www.faithpopcorn.com

198. Brannon, E.L. Fashion forecasting. $2^{\text {nd }}$ ed. Fairchild New York, 2005.

199. Kim, E. et al. Fashion trends analysis and forecasting. Berg London, 2011, pp.56-7.

200. Fashion United. [On-line]. 2018. [Accessed]. Available from:

https://www.fashionunited.com/fashion-news/apparel/wgsn-acquires-stylesight-inc-20132511493656

201. Designers of high fashion enter the age of high tech. [On-line]. 2008. [Accessed]. Available from: https://www.nytimes.com/2008/09/08/technology/08trend.html

202. Worth Global Style Network. [On-line]. 2019. [Accessed]. Available from: https://www.wgsn.com

203. Trendstop. [On-line]. 2019. [Accessed]. Available from: https://www.trendstop.com

204. Diamond, J. and Diamond, E. The world of fashion. $4^{\text {th }}$ ed. Fairchild New York, 2008.

205. Stone, E. The dynamics of fashion. $3^{\text {rd }}$ ed. Fairchild New York, 2008.

206. Feitelberg, R. Trend boards make everything click. Women's Wear Daily, 1998 August 17, p10. In:

Kim, E. et al. Fashion trends analysis and forecasting. Berg London, 2011, pp.145-146.

207. Holland, G. and Jones, R. Fashion trend forecasting. Lawrence King London, 2017, pp.37.

208. Holland, G. and Jones, R. Fashion trend forecasting. Lawrence King London, 2017, pp.36.

209. Vejlgaard, H. Anatomy of a trend. McGraw-Hill New York, 2008, pp.24-27.

210. Vejlgaard, H. Anatomy of a trend. McGraw-Hill New York, 2008, pp.27; 57; 79-80; 116.

211. Vejlgaard, H. Anatomy of a trend. McGraw-Hill New York, 2008, pp.143-145.

212. Meyers, L.S. and Grossen, N.E. Behavioural research. Theory, procedure and design. WH Freeman New York, 1974.

213. Vejlgaard, H. Anatomy of a trend. McGraw-Hill New York, 2008, pp.161.

214. Vejlgaard, H. Anatomy of a trend. McGraw-Hill New York, 2008, pp.188.

215. Frings, G.S. Fashion for concept to consumer. Prentice-Hall New Jersey, 1991.

216. Edelkoort, L. The theories behind colour forecasting. The Briggait Centre, Glasgow. $19^{\text {th }}$ October, 1999 (Presentation). 
217. Brannon, E.L. Fashion forecasting. Fairchild New York, 2000.

218. Perna, R. Fashion forecasting. Fairchild New York, 1987.

219. Levenbach, $\mathrm{H}$. and Cleary, J.P. The beginning forecaster: The forecasting process through data analysis. Lifetime Learning Belmont CA, 1981.

220. Rousso, C. Fashion forward: A guide to fashion forecasting. Fairchild Canada, 2012, pp.7.

221. Verlodt, P. Beyond the crystal ball. In: Colour consulting - A survey of international colour design. Van Nostrand Reinhold New Jersey, 1994, pp.159-163.

222. Wolbers, M.F. Uncovering fashion: Fashion communications across the media. Fairchild New York, 2009, pp.161.

223. Scully, K. and Cobb, D.J. Colour forecasting for fashion: Portfolio skills. Laurence King London, 2012, pp.124.

224. Raymond M. Cited in Scully, K. and Cobb, D.J. Colour forecasting for fashion: Portfolio skills. Laurence King London, 2012, pp.124.

225. Meyers, L.S. and Grossen, N.E. Behavioural research. Theory, procedure and design. WH Freeman New York, 1974.

226. Scully, K. and Cobb, D.J. Colour forecasting for fashion: Portfolio skills. Laurence King London, 2012, pp.22.

227. Holland, G. and Jones, R. Fashion trend forecasting. Lawrence King London, 2017, pp.98.

228. Vejlgaard, H. Anatomy of a trend. McGraw-Hill New York, 2008, pp.142.

229. Edelkoort, L. The theories behind colour forecasting. The Briggait Centre, Glasgow. $19^{\text {th }}$ October, 1999 (Presentation).

230. Frings, G.S. Fashion for concept to consumer. Prentice-Hall New Jersey, 1991, pp.309.

231. Brannon, E.L. and Divita, L. Fashion forecasting. $4^{\text {th }}$ edition. Bloomsbury New York, 2015, pp.1.

232. Fearon, F. Trend forecasting. Knitting International. 1996, 1223(103).

233. Fiore, A.M. and Kimle, P.A. Understanding aesthetics for the merchandising and design professional. Fairchild New York, 1997.

234. Edelkoort, L. The theories behind colour forecasting. The Briggait Centre, Glasgow. $19^{\text {th }}$ October, 1999 (Presentation).

235. Vaughan, F. E. Awakening the intuition. Anchor books New York, 1979, pp.39-48. 
236. Prosch, H. Michael Polanyi: A critical exposition. State University of New York Press Albany, 1986.

237. Peirce, P. The intuitive way: The definitive guide to increasing your awareness. Beyond Words Oregon, 2009.

238. Edelkoort, L. The theories behind colour forecasting. The Briggait Centre, Glasgow. $19^{\text {th }}$ October, 1999 (Presentation).

239. Brannon, E.L. and Divita, L. Fashion forecasting. $4^{\text {th }}$ edition. Bloomsbury New York, 2015, pp.106.

240. Hiers, B. The professional decision thinker. Sidgwick and Jackson London, 1987, pp.111-112.

241. Hiers, B. The professional decision thinker. Sidgwick and Jackson London, 1987, pp.144.

242. Gaimster, J. Visual research methods in fashion. Berg Oxford, 2011, pp.44.

243. Julier, G. The culture of design. Sage Cheltenham, UK, 2000.

244. Lucero, A. and Martens, J.B. Mood boards: industrial designers' perception of using mixed reality. [On-line]. 2005. [Accessed]. Available from https://www.pdfs.semanticscholar.org

245. Garner, S. and McDonagh-Philp, D. Problem interpretation and resolution via visual stimuli: the use of mood boards in design education. International Journal of Art and Design, 2001, 20(1), pp.57-64.

246. Stephdesign. [On-line]. 2005. [Accessed]. Available from:

http://www.stephdesign.com/mobility.htm

247. Boyes, J. Essential fashion design. BT Batsford London, 1998.

248. Kock, K.E. and Domina, T. U4ia for apparel design. Fairchild New York, 2005.

249. Spiekermann. [On-line]. 2005. [Accessed]. Available from: http://www.spiekermann.com/iblog

250. Diane, T. and Cassidy, T. Colour forecasting. Blackwell Oxford UK, 2005.

251. Fiore, A.M. and Kimle, P.A. Understanding aesthetics for the merchandising and design professional. Fairchild New York, 1997.

252. Davis, M. L. Visual design in dress. Second edition. Prentice-Hall New Jersey, 1980.

253. Better product design. Cited in: Cassidy, T.D. The mood board process modelled and understood as a qualitative design research tool. Fashion Practice. 2011, 3(2), pp.225-251.

254. Garner, S. and McDonagh-Philp, D. Problem interpretation and resolution via visual stimuli: the use of mood boards in design education. International Journal of Art and Design, 2001, 20(1), pp.57-64.

255. Bless this mess. [On-line]. 2004. [Accessed]. Available from:

https://www.nytimes.com/2004/02/08/style/bless-this-mess.html 
256. Cassidy, T.D. Mood boards: Current practice in learning and teaching strategies and students' understanding of the process. Fashion Design, Technology \& Education. 2008, 1(1), pp.43-54.

257. Holland, G. and Jones, R. Fashion trend forecasting. Lawrence King London, 2017, pp.91.

258. Holland, G. and Jones, R. Fashion trend forecasting. Lawrence King London, 2017, pp.120.

259. Gale, C. and Kaur, J. The Textile Book. Berg Oxford UK, 2002, pp.140.

260. Granger, M. Fashion. The industry and its careers. Fairchild New York, 2007.

261. McLuckie, T.D. An investigation of colour forecasting. PhD Thesis De Montfort University 2003.

Available from: https://www.core.ac.uk

262. Van Someron, M.W. et al. The think aloud method. A practical guide to modelling cognitive processes. Hartnells Academic Press Cornwall, 1994.

263. Scully, K. and Cobb, D.J. Colour forecasting for fashion: Portfolio skills. Laurence King London, 2012, pp.178.

264. Boeri, C. Colour relationships. In: Colour and design. Berg New York, 2012, pp.35-45.

265. Chiazzari, S. The complete book of colour: Using colour for lifestyle, health and well-being. Harper Collins Dorset, 1998, pp.14.

266. Pulhalla, D.M. Colour: organisational strategies. In: Colour and design. Berg New York, 2012 pp.4660.

267. McLuckie, T.D. An investigation of colour forecasting. PhD Thesis De Montfort University, 2003. Available from: https://www.core.ac.uk

268. Chijiiwa, H. Colour harmony: A guide to creative colour combinations. Edition Olms AG Zurich, 2000.

269. Whelan, B.M. Colour harmony. Rockport Massachusetts, 1994.

270. Cassidy, T.D. Colour Knowledge. In: Textile and clothing design technology. CRC Press Florida 2017, pp.333-356.

271. Perna, R. Fashion forecasting. Fairchild New York, 1987.

272. Edelkoort, L. The theories behind colour forecasting. The Briggait Centre, Glasgow. $19^{\text {th }}$ October, 1999 (Presentation).

273. Brannon, E.L. Fashion forecasting. Fairchild New York, 2000.

274. Kinning, D. Colourcast services. In: Colour consulting - A survey of international colour design. Van Nostrand Reinhold New Jersey, 1994, pp. 174-180. 
275. Rennan, J.G., Burnham, R.W.., \& Newhall, S.M. (1948). Color Terms and Definitions. Psychological Bulletin, 45(3), 2017-230.

276. Holtzschue, L. Understanding colour: an introduction for designers, John Wiley New Jersey, 2011.

277. Puhalla, D.M. Colour: organisational strategies. In: Colour and design. Berg New York, 2012 pp.4660.

278. Klarén, U. and Anter, K.F. Seeing colour. In: Colour and design. Berg New York, 2012, pp.4-8.

279. Harkness, N. The colour wheels of art, perception, science and physiology. Optics and Laser Technology. 38 (2006) pp.219-229

280. DeLong, M. and Martinson, B. Colour and design. Berg New York, 2012. p157.

281. Mottram, J. and Jefferies, T. Colour in the design environment. In: Colour and design. Berg New York, 2012, pp.18-34.

282. Ishihara Test. [On-line]. 2018. [accessed]. Available from:

https://commons.wikimedia.org/wiki/File:Ishihara 9.png).

283. Colour Blindness. [On-line]. 2019. [Accessed]. Available from: https://www.colorblindness.com/ishiharas-test-for-colour-deficiency-38-plates-edition/\#prettyPhoto

284. Wikimedia Commons Ishihara 9. (image) [Online]. 2017. Accessed. Available from: https://commons.wikimedia.org/wiki/File:Ishihara 9.png.

285. Colblindor. Farnsworth-Munsell 100 Hue Colour Vision Test. [On-line]. 2019. [Accessed]. Available from: https://www.color-blindness.com/farnsworth-munsell-100-hue-color-vision-test/.

286. Anderson, A.J. and Johnston, A. W. Test/retest and inter-test agreement of color aptitude measures. Color Research and Application. 40, 3, (2015) pp 224-231

287. Holtzschue, L. Understanding colour: an introduction for designers, John Wiley New Jersey, 2011.

288. Xrite. What is metamerism. [On-line]. 2019. [Accessed]. Available from:

https://www.xrite.com/service-support/what is metamerism.

289. Klarén, U. and Anter, K.F. Seeing colour. In: Colour and design. Berg New York, 2012, pp.3-17.

290. Lambert, P. et al. Color and fibre. Schiffer Atglen PA, 1986, pp.53-54.

291. Eiseman, L. Pantone guide to communicating with colour. Grafix Press Florida, 2000.

292. McLuckie, T.D. An inspirational market research package. MA Thesis Unpublished. 1998, pp.46.

293. Diane, T. and Cassidy, T. Colour forecasting. Blackwell Oxford UK, 2005. 
294. Cassidy, T.D. Colour Knowledge. In: Textile and clothing design technology. CRC Press Florida 2017, pp.333-356.

295. Keiser, S.J and Garner, M.B. Beyond design: The synergy of apparel product development. $3^{\text {rd }}$ edition. Bloomsbury New York, 2015, pp.138.

296. Agarwal, N. (2003) A note on colour inconstancy. Cited in: Keiser, S.J and Garner, M.B. Beyond design: The synergy of apparel product development. $3^{\text {rd }}$ edition. Bloomsbury New York, 2015, pp.1-4.

297. Whelen, B.M. Colour harmony 2. Rockport Massachusetts, 1994, pp.8-11.

298. Lloyd, D. The colour book. Craft Print International Singapore, 2007, pp.18-19.

299. Lambert, P. et al. Color and fibre. Schiffer Atglen PA, 1986, pp.25.

300. Cassidy, T.D. Colour Knowledge. In: Textile and clothing design technology. CRC Florida, 2017, pp.333-356, pp.354.

301. Wilcox, M. Blue and yellow don't make green. Imago Productions Singapore, 2009, pp.23.

302. Lambert, P. et al. Color and fibre. Schiffer Atglen PA, 1986, pp.40.

303. Cassidy, T.D. Colour Knowledge. In: Textile and clothing design technology. Florida, 2017, pp.333356, pp.353.

304. Cassidy, T.D. Colour Knowledge. In: Textile and clothing design technology. CRC Florida, 2017, pp.333-356.

305. Wilcox, M. Blue and yellow don't make green. Imago Productions Singapore, 2009.

306. Lloyd, D. The colour book. Craft Print International Singapore, 2007.

307. Wilcox, M. Blue and yellow don't make green. Imago Productions Singapore, 2009, pp.7-8.

308. Fashion Coordinator. [On-line]. 2017. [Accessed]. Available from:

https://www.telegraph.co.uk/fashion/style/colour-wheel-basic-guide-clothing-colour-matching/

309. Live about. [On-line]. 2017. [Accessed]. Available from: https://www.liveabout.com/fashionneutrals-2658077.

310. Danger, E.P. Using colour to sell. Gower Aldershot UK, 1968, pp.19.

311. Lambert, P. et al. Color and fibre. Schiffer Atglen PA, 1986, pp.31.

312. Dunn-Edwards Paints. [On-Line]. 2019. [Accessed]. Available from:

https://www.dunnedwards.com/colors/specs/posts/color-terminology-hues-tints-shades-and-tones.

313. Danger, E.P. Using colour to sell. Gower Aldershot UK, 1968, pp.20-21. 
314. Keiser, S.J and Garner, M.B. Beyond design: The synergy of apparel product development. $3^{\text {rd }}$ edition. Bloomsbury New York, 2015, pp.140.

315. Danger, E.P. Using colour to sell. Gower Aldershot UK, 1968, pp.19.

316. Danger, E.P. Using colour to sell. Gower Aldershot UK, 1968, pp.23.

317. Boeri, C. Colour relationships. In: Colour and design. Berg New York, 2012, pp.35-36.

318. DeLong, M. and Martinson, B. Colour and design. Berg New York, 2012, pp.2.

319. John Ruskin. [On-line]. 2017. [Accessed]. Available from:

https://www.tate.org.uk/art/artists/john-ruskin-465;

320. Boeri, C. Colour relationships. In: Colour and design. Berg New York, 2012, pp.35-36.

321. Whelan, B.M. Colour harmony. Rockport Massachusetts, 1994, pp.23.

322. McLuckie, T.D. Colour harmony in knitwear design and colour forecasting for the millennium. BSc. (Hons) Dissertation Unpublished, 1998.

323. Danger, E.P. Using colour to sell. Gower Aldershot UK, 1968, pp.54.

324. Wonderful colours. [On-line]. 2019. [Accessed]. Available from:

http://www.wonderfulcolors.org/blog/birren-color-theory/.

325. Klarén, U. and Anter, K.F. Seeing colour. In: Colour and design. Berg New York, 2012, pp.8.

326. Itten, J. The art of color. The subjective experience and objective rationale of colour. Wiley New Jersey, 1961, pp.36.

327. McLuckie, T.D. Colour harmony in knitwear design and colour forecasting for the millennium. BSc. (Hons) Dissertation Unpublished, 1998.

328. Agrawal, N., and Singh, S., Role of product Color in consumer behaviour. IOSR Journal of Business \& Management. http://www.iosrjournals.org/iosr-jbm/papers/Conf.17001-2017/Volume-1/6.\%2033$\underline{38 . p d f}$

329. Burns, L.D. et al. The business of fashion: Designing, manufacturing and marketing. Fairchild New York, 2011, pp.112.

330. Keiser, S.J and Garner, M.B. Beyond design: The synergy of apparel product development. $3^{\text {rd }}$ edition. Bloomsbury New York, 2015, pp.249.

331. Danger, E.P. Using colour to sell. Gower Aldershot UK, 1968, pp.4.

332. Keiser, S.J and Garner, M.B. Beyond design: The synergy of apparel product development. $3^{\text {rd }}$ edition. Bloomsbury New York, 2015, pp.249-250. 
333. How to be a successful retailer. Colouring customers. Drapers Record. 1998, 23, pp.i-ii.

334. Keiser, S.J and Garner, M.B. Beyond design: The synergy of apparel product development. $3^{\text {rd }}$ edition. Bloomsbury New York, 2015, pp.135-136.

335. Puhalla, D.M. Colour: organisational strategies. In: Colour and design. Berg New York, 2012 pp.49.

336. Keiser, S.J and Garner, M.B. Beyond design: The synergy of apparel product development. $3^{\text {rd }}$ edition. Bloomsbury New York, 2015, pp.132-4.

337. GretagMacbeth. Fundamentals of colour and appearance. New Windsor New York, 1998.

338. Boeri, C. Colour relationships. In: Colour and design. Berg New York, 2012, pp.39.

339. The Nobel Prize. Wilhelm Ostwald biographical. [On-line]. 2019. [Accessed]. Available from: https://www.nobelprize.org/nobel prizes/chemistry/laureates/1909/ostwald-bio.html .

340. Puhalla, D.M. Colour: organisational strategies. In: Colour and design. Berg New York, 2012 pp.4660, pp.46-49.

341. Mottram, J. and Jefferies, T. Colour in the design environment. In: Colour and design. Berg New York, 2012, pp.23.

342. International Commission on illumination. [On-line]. 2019. [Accessed]. Available from: http://www.cie.co.at/technical-work/divisions/division1)

343. British Standard. [On-line]. 2019. [Accessed]. Available from:

https://www.britishstandardscolour.com

344. Pantone. [On-line]. 2019. [Accessed]. Available from: https://www.pantone.com/about-pantone

345. How Pantone became the definitive language of colour. [On-line]. 2015. [Accessed]. Available from: https://www.fastcompany.com/3050240/how-pantone-became-the-definitive-language-of-color

346. Keiser, S.J and Garner, M.B. Beyond design: The synergy of apparel product development. $3^{\text {rd }}$ edition. Bloomsbury New York, 2015, pp.148.

347. Pantone. [On-line]. 2019. [Accessed]. Available from: https://www.pantone.com/about-pantone

348. NCS Color. [On-line]. 2019. [Accessed]. Available from: https://www.ncscolour.com

349. Tate, S.L. Inside fashion design. $2^{\text {nd }}$ edition. Harper and Row New York, 1984.

350. Spillane, M. The complete style guide from the Color Me Beautiful organisation. Piatkus London, 1995.

351. Jacques, B. The complete colour, style and image book. Thorsons California, 1994. 
352. Rath, P.M et al. Marketing fashion. A global perspective. Bloomsbury Canada, 2012, pp.355.

353. Brannon, E.L. Fashion forecasting. Fairchild New York, 2000, pp.126.

354. Nippon Color \& Design Research Institute, Inc. Colour and image forecasting. In Linton 1994: 26 In: Colour consulting - A survey of international colour design. Van Nostrand Reinhold New Jersey, 1994, pp.25-31.

355. Keiser, S.J and Garner, M.B. Beyond design: The synergy of apparel product development. $3^{\text {rd }}$ edition. Bloomsbury New York, 2015, pp.118.

356. Levenbach, $\mathrm{H}$. and Cleary, J.P. The beginning forecaster: The forecasting process through data analysis. Lifetime Learning Belmont CA, 1981.

357. Rousso, C. Fashion forward: A guide to fashion forecasting. Fairchild Canada, 2012, pp.7.

358. Rousso, C. Fashion forward: A guide to fashion forecasting. Fairchild Canada, 2012, pp.104.

359. Keiser, S.J and Garner, M.B. Beyond design: The synergy of apparel product development. $3^{\text {rd }}$ edition. Bloomsbury New York, 2015, pp.120.

360. McAssey, J. and Buckley, C. Basics fashion design 08: Styling. AVA Lausanne Switzerland, 2011, pp.121.

361. Rousso, C. Fashion forward: A guide to fashion forecasting. Fairchild Canada, 2012, pp.100.

362. Brannon, E.L. Fashion forecasting. Fairchild New York, 2000.

363. Rousso, C. and Ostroff, N.K. Fashion Forward. Bloomsbury New York, 2018, pp.75.

364. Sproles, G.B. Fashion. Consumer behaviour towards dress. Burgess Minneapolis, 1979.

365. Meadows, T. How to set up and run a fashion label. Laurence King London, 2009, pp.92.

366. Raymond, M. The trend forecaster's handbook. Laurence King London, 2010, pp.17.

367. Blythe, J. Essentials of marketing. $5^{\text {th }}$ edition. Pearson Essex, 2012, pp.123.

368. Holland, G. and Jones, R. Fashion trend forecasting. Lawrence King London, 2017, pp.50.

369. Keiser, S.J and Garner, M.B. Beyond design: The synergy of apparel product development. $3^{\text {rd }}$ edition. Bloomsbury New York, 2015, pp.104.

370. Rousso, C. Fashion forward: A guide to fashion forecasting. Fairchild Canada, 2012, pp.101-104.

371. Easey. M. Fashion marketing. Blackwell Oxford, 1995.

372. Sustainable Clothing Action Plan (SCAP). WRAP. [On-line]. 2019. [Accessed]. Available from: http://www.wrap.org.uk/sites/files/wrap/Strategic\%20loop\%20-\%20textiles.pdf. 
373. A new textiles economy: Redesigning fashion's future. Ellen MacArthur Foundation (2017). [Online]. 2019. [Accessed]. Available from: http://www.ellenmacarthurfoundation.org/publications.

374. Davis, F. Fashion, Culture and Identity. University of Chicago Press London, 1994, pp.157.

375. Keiser, S.J and Garner, M.B. Beyond design: The synergy of apparel product development. $3^{\text {rd }}$ edition. Bloomsbury New York, 2015, pp.104s.

376. Raymond, M. The trend forecaster's handbook. Laurence King London, 2010, pp.18.

377. Meadows, T. How to set up and run a fashion label. Laurence King London, 2009, pp.92.

378. Holland, G. and Jones, R. Fashion trend forecasting. Lawrence King London, 2017, pp.50.

379. Meadows, T. How to set up and run a fashion label. Laurence King London, 2009, pp.93-94.

380. Blythe, J. Essentials of marketing. $5^{\text {th }}$ edition. Pearson Essex, 2012, pp.194.

381. Brannon, E.L. and Divita, L. Fashion forecasting. $4^{\text {th }}$ edition. Bloomsbury New York, 2015, pp.50.

382. Raymond, M. The trend forecaster's handbook. Laurence King London, 2010, pp.18.

383. Keiser, S.J and Garner, M.B. Beyond design: The synergy of apparel product development. $3^{\text {rd }}$ edition. Bloomsbury New York, 2015, pp.104.

384. Bye, E. Fashion design. Berg Oxford, 2010, pp.2.

385. Meadows, T. How to set up and run a fashion label. Laurence King London, 2009, pp.92.

386. Brannon, E.L. Designer's guide to fashion apparel. Fairchild New York, 2011, pp.121.

387. Meadows, T. How to set up and run a fashion label. Laurence King London, 2009, pp.92.

388. Raymond, M. The trend forecaster's handbook. Laurence King London, 2010, pp.20.

389. Brannon, E.L. and Divita, L. Fashion forecasting. $4^{\text {th }}$ edition. Bloomsbury New York, 2015, pp.50.

390. Keiser, S.J and Garner, M.B. Beyond design: The synergy of apparel product development. $3^{\text {rd }}$ edition. Bloomsbury New York, 2015, pp.104.

391. Bye, E. Fashion design. Berg Oxford, 2010, pp.30.

392. Raymond, M. The trend forecaster's handbook. Laurence King London, 2010, pp.20.

393. Brannon, E.L. and Divita, L. Fashion forecasting. $4^{\text {th }}$ edition. Bloomsbury New York, 2015, pp.50.

394. Meadows, T. How to set up and run a fashion label. Laurence King London, 2009, pp.92.

395. Raymond, M. The trend forecaster's handbook. Laurence King London, 2010, pp.26. 
396. Holland, G. and Jones, R. Fashion trend forecasting. Lawrence King London, 2017, pp.50.

397. Meadows, T. How to set up and run a fashion label. Laurence King London, 2009, pp.68.

398. Meadows, T. How to set up and run a fashion label. Laurence King London, 2009, pp.92.

399. Kim, E. et al. Fashion trends analysis and forecasting. Berg London, 2011, pp.xiii.

400. Sproles, G.B. Analyzing fashion life cycles: Principles and perspectives. Journal of Marketing. 1981, 45(4), pp.116-124.

401. Cassidy, T.D. Sustainable colour forecasting. In: Sustainability in Fashion and Textiles. Greenleaf Sheffield UK, 2013, pp.111-124.

402. Frings. G.S. Fashion concept to consumer. $2^{\text {nd }}$ edition. Pearson Essex, 1996, pp.63.

403. Solomon, M. and Rabolt, N. Consumer behaviour in fashion. Prentice Hall New Jersey, 2004, pp.11.

404. Yurchisin, J. and Johnson, K.P. fashion and the consumer. Berg London, 2010, pp.62.

405. Niinimäki, K. and Hassi, L. Emerging Design Strategies for sustainable production and consumption of textiles and clothing. Journal of Cleaner Production. 2011, 19(16), pp.1876-1883.

406. Bulow, J. An economic theory of planned obsolescence. Quarterly journal of Economics. 1986, 101(4), pp.729-750.

407. Cassidy, T.D. Sustainable colour forecasting. In: Sustainability in Fashion and Textiles. Greenleaf Sheffield UK, 2013, pp.111-124.

408. Planned Obsolescence. [On-line]. 2009. [Accessed]. Available from:

https://www.economist.com/news/2009/03/23/planned-obsolescence

409. Cassidy, T.D. Conceptualizing Sustained High Quality Fashion Products in a Devalue Dominated Marketplace. Fashion Practice. 2017, 9(2), pp.235-253.

410. Kim, E. et al. Fashion trends analysis and forecasting. Berg London, 2011, pp.90.

411. Fiore, A.M. Understanding aesthetics for the merchandising and design professional. $2^{\text {nd }}$ edition. Fairchild New York, 2010, pp.17.

412. Niinimäki, K. Sustainable consumer satisfaction in the context of clothing. In: Product-service system design for sustainability. Greenleaf Sheffield, 2011.

413. Cassidy, T.D. Sustainable colour forecasting. In: Sustainability in Fashion and Textiles. Greenleaf Sheffield UK, 2013, pp.111-124.

414. Sproles, G.B. Fashion. Consumer behaviour towards dress. Burgess Minneapolis, 1979. 
415. Waldman, M. A new perspective on planned obsolescence. The Quarterly Journal of Economics. 108(1), 1993, pp.273-283.

416. Blythe, J. Essentials of marketing. $5^{\text {th }}$ edition. Pearson Essex, 2012, pp.223-224.

417. Meadows, T. How to set up and run a fashion label. Laurence King London, 2009.

418. Rousso, C. and Ostroff, N.K. Fashion Forward. Bloomsbury New York, 2018, pp.71.

419. Meadows, T. How to set up and run a fashion label. Laurence King London, 2009, pp.95.

420. McAssey, J. and Buckley, C. Basics fashion design 08: Styling. AVA Lausanne Switzerland, 2011, pp.118.

421. Kim, E. et al. Fashion trends analysis and forecasting. Berg London, 2011, pp.19.

422. McCracken, G. Culture and consumption. Indiana university Press Bloomington IN, 1985.

423. Cadigan, E. Sourcing and selecting textiles for fashion. Bloomsbury London, 2014.

424. McAssey, J. and Buckley, C. Basics fashion design 08: Styling. AVA Lausanne Switzerland, 2011, pp.118.

425. Chowdhary, U. Shifting Fashion Paradigm: From Status Quo to Mostly Business. American International Journal of Social Science. 2015, 4(6), pp.40-45.

426. Kim, E. et al. Fashion trends analysis and forecasting. Berg London, 2011, pp.58.

427. Vejlgaard, H. Anatomy of a trend. McGraw-Hill New York, 2008, pp.64-65.

428. McAssey, J. and Buckley, C. Basics fashion design 08: Styling. AVA Lausanne Switzerland, 2011, pp.21.

429. Keiser, S.J and Garner, M.B. Beyond design: The synergy of apparel product development. $3^{\text {rd }}$ edition. Bloomsbury New York, 2015, pp.120.

430. Wolbers, M.F. Uncovering fashion: Fashion communications across the media. Fairchild New York, 2009, pp.133.

431. Stone, E. The dynamics of fashion. $3^{\text {rd }}$ ed. Fairchild New York, 2008.

432. Rath, P.M et al. Marketing fashion. A global perspective. Bloomsbury Canada, 2012, pp.253.

433. Sproles, G.B. Analyzing fashion life cycles: Principles and perspectives. Journal of Marketing. 1981, 45(4), pp.116-124.

434. Brannon, E.L. Fashion forecasting. Fairchild New York, 2000. 
435. Niinimäki, K. and Hassi, L. Emerging Design Strategies for sustainable production and consumption of textiles and clothing. Journal of Cleaner Production. 2011, 19(16), pp.1876-1883.

436. Lamb, M. Trends in color and pattern. In: Colour consulting - A survey of international colour design. Van Nostrand Reinhold New Jersey, 1994, pp.63-65, pp.63.

437. Oberascher, L. Cyclic recurrence of collective color preferences. In: Colour consulting - A survey of international colour design. Van Nostrand Reinhold New Jersey, 1994, pp.66-77.

438. Charbonneau, K.X. Color forecasting - mystery or science? In: Colour consulting - A survey of international colour design. Van Nostrand Reinhold New Jersey, 1994, pp.101-114.

439. Brannon, E.L. Fashion forecasting. Fairchild New York, 2000.

440. Jones, R.M. The apparel industry. Blackwell Oxford, 2002.

441. Rousso, C. and Ostroff, N.K. Fashion Forward. Bloomsbury New York, 2018, pp.133.

442. Stansfield, J. and Whitfield, T.W. Can future colour trends be predicted on the basis of past colour trends? An empirical investigation. Color Research \& Application. 2005, 30(3), pp.235-242.

443. Kim, E. et al. Fashion trends analysis and forecasting. Berg London, 2011, pp.59.

444. Rousso, C. Fashion forward: A guide to fashion forecasting. Fairchild Canada, 2012, pp.194.

445. Radeloff, D.J. psychological types, color attributes and color preferences of clothing, textiles and design students. Clothing and Textiles Research Journal. 1991, 9(3), pp.59-67.

446. Cadigan, E. Sourcing and selecting textiles for fashion. Bloomsbury London, 2014, pp.213.

447. Meadows, T. How to set up and run a fashion label. Laurence King London, 2009, pp.90.

448. Great British Speakers. [On-line]. 2019. [Accessed]. Available from:

https://www.thefuturelaboratory.com/blog/author/martin-raymond

449. Raymond, M. The trend forecaster's handbook. Laurence King London, 2010, pp.4.

450. Duggan, W. Strategic intuition: The creative spark in Human achievement. Columbia Business School Publishing New York, 2013, pp.1-2.

451. Kolb, B. and Whishaw, I.Q. Fundamentals of human neuropsychology. $7^{\text {th }}$ edition. Worth Publishers New York, 2015.

452. Aunger, R. Darwinizing culture: The status of memetics as a science. Oxford University Press Oxford, 2001.

453. Futurism - the trendiest profession. [On-line]. 2019. [Accessed]. Available from: https://www.questia.com/magazine/1G1-20324617/futurism-the-trendiest-profession 
454. Mbonu, E. Fashion design research. Laurence King London, 2014, pp.70-71.

455. Blythe, J. Essentials of marketing. $5^{\text {th }}$ edition. Pearson Essex, 2012, pp..32.

456. Naisbitt, J. Megatrends: Ten new directions transforming our lives. Grand Central New York, 1982.

457. Naisbitt. [On-line]. 2019. [Accessed]. Available from: https://www.naisbitt.com

458._Rath, P.M et al. Marketing fashion. A global perspective. Bloomsbury Canada, 2012, pp.43-58.

459. Granger, M. Fashion. The industry and its careers. Fairchild New York, 2007, pp.4.

460. Angus, I. and Jhally, S. Cultural politics in contemporary America. Routledge New York, 1989.

461. Gans, H. Popular culture and high culture. Basic Books New York, 1974.

462. Brannon, E.L. and Divita, L. Fashion forecasting. $4^{\text {th }}$ edition. Bloomsbury New York, 2015, pp.10.

463. O'Neill, J. Forecasting - Fact or Fiction? Textile Horizons. 1989. 9(9), pp.26-8.

464. Wolbers, M.F. Uncovering fashion: Fashion communications across the media. Fairchild New York, 2009, pp.105-106.

465. The Future Laboratory. [On-line]. 2019. [Accessed]. Available from:

https://www.thefuturelaboratory.com ]

466. Brannon, E.L. and Divita, L. Fashion forecasting. $4^{\text {th }}$ edition. Bloomsbury New York, 2015, pp.108.

467. Brannon, E.L. and Divita, L. Fashion forecasting. $4^{\text {th }}$ edition. Bloomsbury New York, 2015, pp.110.

468. Raymond, M. The trend forecaster's handbook. Laurence King London, 2010.

469. Churchill, G.A. and Lacobucci, D. Market research: Methodological foundations. Earlie Lite Books Nashville, 2005.

470. Hague, P. Market research: A guide to planning, methodology and evaluation. Kogan Page London, 2003, pp.67-68.

471. Silver, L. et al. The essentials of marketing research. $3^{\text {rd }}$ Ed. Routledge New York, 2013, pp.44.

472. Levy, M. and Weitz, B.A. Retailing management. $7^{\text {th }}$ edition. McGraw-Hill New York, 2009.

473. Raymond, M. The trend forecaster's handbook. Laurence King London, 2010, pp.467.

474. Rath, P.M et al. Marketing fashion. A global perspective. Bloomsbury Canada, 2012, pp.151.

475. Bryman, A. Social research methods. $4^{\text {th }}$ ed. Oxford University Press Oxford, 2012, pp.711.

476. Bryman, A. Social research methods. $4^{\text {th }}$ ed. Oxford University Press Oxford, 2012, pp.469. 
477. Edelkoort, L. The theories behind colour forecasting. The Briggait Centre, Glasgow. $19^{\text {th }}$ October, 1999 (Presentation).

478. Silver, L. et al. The essentials of marketing research. $3^{\text {rd }}$ Ed. Routledge New York, 2013, pp.66.

479. Diane, T. and Cassidy, T. Colour forecasting. Blackwell Oxon UK, 2005, pp.106-7.

480. Cassidy, T, D. Personal colour analysis, consumer colour preferences and colour forecasting for the fashion and textile industries. Colour: Design \& Creativity. 2007, 1(1), pp.1-14.

481. Cassidy, T.D. Sustainable colour forecasting. In: Sustainability in Fashion and Textiles. Greenleaf Sheffield UK, 2013, pp.111-124.

482. Cassidy, T.D. Consumer colour and style preferences: A new approach to tackling the textile waste conundrum. In: Eco-friendly and fair. Routledge Oxon UK, 2018, pp.83-91.

483. McLuckie, T.D. An investigation of colour forecasting. PhD Thesis De Montfort University, 2003. Available from: https://www.core.ac.uk

484. Schloss, K.B. et al. Modeling color preference using color space metrics. Vision Research. 2018, 151, pp.99-116.

485. Schloss, K.B. et al. Seasonal variations in colour preferences. Cognitive Science. 2017, 41, pp.15891612.

486. Eysenck, H.J. Type-factors in aesthetic judgements. British Journal of Psychology. 1941, 31(3), pp.262-270.

487. Porter, T. Color in the looking glass. In: Colour consulting - A survey of international colour design. Van Nostrand Reinhold New Jersey, 1994, pp.1-9.

488. Wilcox, M. Blue and yellow don't make green. Imago Productions Singapore, 2009.

489. Lloyd, D. The colour book. Craft Print International Singapore, 2007.

490. Wright, A. The beginner's guide to colour psychology. Kyle Cathie London, 1998.

491. Color psychology: How color meaning affect your brand. [On-line]. 2018. [Accessed]. Available from: https://www.oberlo.co.uk

492. Gans, H. Popular culture and high culture. Basic Books New York, 1974.

493. Dorling Kindersley. Signs and symbols: An illustrated guide to their origins and meanings. Dorling Kindersley London, 2008.

494. A new textiles economy: Redesigning fashion's future. Ellen MacArthur Foundation (2017). [Online]. 2019. [Accessed]. Available from: http://www.ellenmacarthurfoundation.org/publications. 
495. Bryman, A. Social research methods. $4^{\text {th }}$ ed. Oxford University Press Oxford, 2012. 